\title{
Rigidity of the 1-Bakry-Émery inequality and sets of finite perimeter in RCD spaces
}

\author{
Luigi Ambrosio * $\quad$ Elia Brué ${ }^{\dagger} \quad$ Daniele Semola ${ }^{\ddagger}$
}

September 12, 2019

\begin{abstract}
This note is dedicated to the study of the asymptotic behaviour of sets of finite perimeter over $\operatorname{RCD}(K, N)$ metric measure spaces. Our main result asserts existence of a Euclidean tangent half-space almost everywhere with respect to the perimeter measure and it can be improved to an existence and uniqueness statement when the ambient is non collapsed. As an intermediate tool, we provide a complete characterization of the class of $\operatorname{RCD}(0, N)$ spaces for which there exists a nontrivial function satisfying the equality in the 1-Bakry-Émery inequality. This result is of independent interest and it is new, up to our knowledge, even in the smooth framework.
\end{abstract}

\section{Contents}

1 Preliminaries 4

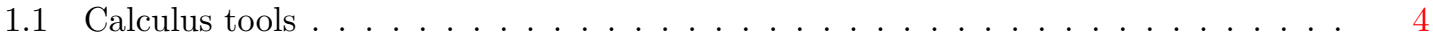

$1.2 \operatorname{RCD}(K, N)$ metric measure spaces $\ldots \ldots \ldots \ldots \ldots \ldots \ldots$

1.3 Convergence and stability results for sequences of $\operatorname{RCD}(K, N)$ spaces . . . . . . . 11

2 Rigidity of the 1-Bakry-Émery inequality and splitting theorem 13

3 Convergence and stability results for sets of finite perimeter 20

4 Tangents to sets of finite perimeter in $\operatorname{RCD}(K, N)$ spaces 26

A Appendix 33

\section{Introduction}

In recent years the theory of metric measure spaces $(X, \mathrm{~d}, \mathfrak{m})$ (in short, m.m.s.) satisfying, in a weak sense, upper bounds on dimension and lower bounds on the Ricci tensor has undergone fast and remarkable developments, see [A18] for a recent survey on this topic. In this paper we focus on the $\mathrm{CD}(K, N)$ theory pioneered by Lott-Villani and Sturm. More specifically, we are concerned with the "Riemannian" side of the theory, the class of $\operatorname{RCD}(K, N)$ (with $K \in \mathbb{R}, 1 \leq N<+\infty$ ) m.m.s. proposed in [G15] after the study of their infinite-dimensional counterparts $\operatorname{RCD}(K, \infty)$ in [AGS14]. The first results providing the equivalence between $\operatorname{RCD}(K, \infty)$ and the validity of Bochner's inequality were obtained in [AGS15], then [EKS15] estabilished the equivalence with the dimensional Bochner inequality for the so-called class $\operatorname{RCD}^{*}(K, N)$. Eventually, full equivalence between $\operatorname{RCD}^{*}(K, N)$ and $\operatorname{RCD}(K, N)$ has been achieved, among other things, in [CM16]), closing the circle. By now the class of $\operatorname{RCD}(K, N)$ spaces is known to include the so-called Ricci limit spaces of the Cheeger-Colding theory, Alexandrov spaces [P11] and other singular spaces.

*Scuola Normale Superiore, luigi.ambrosio@sns.it.

†Scuola Normale Superiore, elia.brue@sns.it.

${ }^{\ddagger}$ Scuola Normale Superiore, daniele.semola@sns.it. 
Many structural properties of $\operatorname{RCD}(K, N)$ spaces have been established in the recent years, in most cases after their proof in the setting of Ricci limit spaces, and sometimes with essentially new strategies of proof, given the absence of a smooth approximation. Among these structural results it is worth mentioning the splitting theorem [G13], the second-order calculus [G18], the existence of an "essential dimension" [BS18], the rectifiability as metric measure spaces [MN14, KM18, DPMR17, GP16a], the validity of sharp heat kernel bounds [JLZ14], the well-posedness of ODE's associated to Sobolev vector fields [AT14].

At this stage of the development of the RCD theory, it is natural to investigate the typical themes of Geometric Measure Theory, since GMT provides techniques for dealing with nonsmooth objects already when the ambient space is smooth. One of the most fundamental results of GMT, that eventually led to the Federer-Fleming theory of currents [FF60], is De Giorgi's structure theorem for sets $E$ of finite perimeter. De Giorgi's theorem, established in [DG54, DG55], provides the representation

$$
\left|D \chi_{E}\right|=\mathscr{H}^{n-1}\llcorner\mathcal{F} E
$$

of the perimeter measure $\left|D \chi_{E}\right|$ as the restriction of $\mathscr{H}^{n-1}$ to a suitable measure-theoretic boundary $\mathcal{F} E$ of $E$. In addition, it provides a description of $E$ on small scales, showing that for all $x \in \mathcal{F} E$ the rescaled set $r^{-1}(E-x)$ is close, for $r>0$ sufficiently small, to an halfspace orthogonal to $\nu_{E}(x)$.

Our goal in this paper is to provide an extension of this result to the setting of $\operatorname{RCD}(K, N)$ m.m.s. In the study of this problem, we realized the importance of the study of the rigidity case in the Bakry-Émery inequality, namely the analysis of the implications of the condition

$$
\left|\nabla P_{t} f\right|=P_{t}|\nabla f| \quad \mathfrak{m} \text {-a.e. in } X, \text { for every } t \geq 0
$$

for some nontrivial function $f$, if the ambient space is $\operatorname{RCD}(0, N)$.

Since the study of (0.1) has an independent interest, we first illustrate our result in this direction, then we move back to sets of finite perimeter. Our rigidity result, stated in Theorem 2.1 , shows that (0.1) is sufficiently strong to imply the splitting of the m.m.s. as $Z \times \mathbb{R}$, in addition with a monotonic dependence on $f$ on the split real variable. This result could be considered as "dual" to the classical splitting theorem, since the basic assumption is not the existence of a curve with a special property (namely an entire geodesic), but rather the existence of a function satisfying (0.1). However, our proof builds on Gigli's splitting theorem and is achieved in these steps:

1 ) by first variations in $(0.1)$ we prove that the unit vector fields $b_{t}=\nabla P_{t} f /\left|\nabla P_{t} f\right|$ are independent of $t$, divergence-free and with symmetric part of derivative in $L^{2}$, in a suitable weak sense;

2) because of this, the theory of flows developed in [AT14] applies, and provides a measurepreserving semigroup of isometries $\boldsymbol{X}_{t}$;

$3)$ we use $\boldsymbol{X}_{t}$ to show in Proposition 2.11 that $\left(P_{s} f\right) \circ \boldsymbol{X}_{-t}$ is a value function, more precisely

$$
P_{s} f\left(\boldsymbol{X}_{-t}(x)\right)=\min _{\bar{B}_{t}(x)} P_{s} f \quad \forall x \in X, s>0, t \geq 0 .
$$

In the proof of this fact we have been inspired by the analysis of isotropic Hamilton-Jacobi equations made in [N14] (see also [AF14]), even though our proof is self-contained. Using this representation of $\left(P_{s} f\right) \circ \boldsymbol{X}_{-t}$ it is not hard to prove that all flow curves $t \mapsto \boldsymbol{X}_{t}(x)$ are lines and, in particular, Gigli's theorem [G13, G14] applies. Even though this refinement does not play a role in the second part of the paper, we also prove that the validity of $\left|\nabla P_{t} f\right|=P_{t}|\nabla f|$ for some $t>0$ implies the validity for all $t \geq 0$, namely (0.1).

Now, what is the relation between (0.1) and the fine structure of sets of finite perimeter? In De Giorgi's proof and its many extensions to currents and other complex objects, the normal direction $\nu_{E}$ coming out of the blow-up analysis is identified by looking at the polar decomposition $D \chi_{E}=\nu_{E}\left|D \chi_{E}\right|$ of the distributional derivative (choosing approximate continuity points of $\nu_{E}$, relative to $\left.\left|D \chi_{E}\right|\right)$. In turn, the polar decomposition essentially depends on the particular structure of the tangent bundle of the Euclidean space. In the RCD theory, as in Cheeger's theory of PI 
spaces (see [GP16b] for a deeper discussion of the relation between the two notions of tangent bundle), the tangent bundle is defined only up to $\mathfrak{m}$-negligible sets, not in a pointwise sense. So, it could in principle be used to write a polar decomposition analogous to the Euclidean theory only for vector-valued (in a suitable sense) measures absolutely continuous w.r.t. $\mathfrak{m}$. We bypass this difficulty by establishing this new principle: at $\left|D \chi_{E}\right|$-a.e. point $x$, any tangent set $F$ to $E$ at $x$ in any tangent, pointed, metric measure structure $(Y, \varrho, \mu, y)$ has to satisfy the condition

$$
\left|\nabla P_{t} \chi_{F}\right| \mu=P_{t}^{*}\left|D \chi_{F}\right| \quad \forall t \geq 0 \text {. }
$$

Notice that $\left|D \chi_{F}\right|$, the semigroup $P_{t}$ and its dual $P_{t}^{*}$ in $(0.2)$ have, of course, to be understood in the tangent metric measure structure. The proof of this principle, given in Theorem 4.2, ultimately relies on the lower semicontinuity of the perimeter measure $\left|D \chi_{E}\right|$ (as it happens for the powerful principle that lower semicontinuity and locality imply asymptotic local minimality, see [F66, W89], and [C99]) and gradient contractivity. From (0.2), gradient contractivity easily yields that all functions $f=P_{s} \chi_{F}$ satisfy (0.1); this leads to a splitting both of $(Y, \varrho, \mu)$ and $F$, and to the identification of a "tangent halfspace" $F$ to $E$ at $x$.

Using these ideas we can prove the following structure results for sets of finite perimeter $E$ in $\operatorname{RCD}(K, N)$ m.m.s.: first, in Theorem 4.3, we prove that $E$ admits a Euclidean half-space as tangent at $x$ for $\left|D \chi_{E}\right|$-a.e. $x \in X$. This result becomes more precise in the setting of noncollapsed $\operatorname{RCD}(K, N)$ m.m.s. of [DPG18]: in this case we prove in Corollary 4.4 and Corollary 4.7 that $\left|D \chi_{E}\right|$ is concentrated on the $N$-dimensional regular set of the ambient metric measure structure and we provide the representation formula $\left|D \chi_{E}\right|=\mathcal{S}^{N-1}\left\llcorner\mathcal{F} E\right.$ (where $\mathcal{S}^{k}$ denotes the $k$-dimensional spherical Hausdorff measure, see also (4.4) for the precise definition of $\mathcal{F} E$ ), for an appropriate notion of measure-theoretic boundary $\mathcal{F} E$.

The paper is organized as follows. After the preliminary section 1, where we collect basic notation, calculus rules and basic facts about $\operatorname{RCD}(K, N)$ spaces, Sobolev and BV functions and flows associated to vector fields, in section 2 we prove our rigidity results, allong the lines described above. We dedicate section 3 to the study of the behaviour of sequences of sets $E_{i}$ in m.m.s. $\left(X_{i}, \mathrm{~d}_{i}, \mathfrak{m}_{i}\right)$ convergent in the measured Gromov-Hausdorff sense to $(X, \mathrm{~d}, \mathfrak{m})$. In particular, using appropriate notions of compactness for sequences of functions and measures in varying metric measure structures, we focus on compactness and lower semicontinuity of the perimeter measure. We apply these results in section 4 , where we specialize our analysis to the case when $\left(X_{i}, \mathrm{~d}_{i}, \mathfrak{m}_{i}\right)$ arise from the rescaling of a pointed m.m.s. This theme is also investigated in [EGLS18], but in our paper we take advantage of the curvature/dimension bounds to establish the stronger rigidity property (0.2) satisfied by tangent sets $F$ in the tangent metric measure structure. Then, using the splitting property and the principle that "tangents to a tangent are tangent", we are able to recover the above-mentioned structure results of sets of finite perimeter. Finally, Appendix A is devoted to a self-contained proof of the iterated tangents principle, adapting the argument of Preiss' seminal paper [P87] (see also [AKL08, GMR15]).

A natural question arising from the results and methods in this paper is the rectifiability of the measure-theoretic boundary $\mathcal{F} E$. Our blow up-analysis shows that the measure $\left|D \chi_{E}\right|=$ $\mathcal{S}^{N-1}\left\llcorner\mathcal{F} E\right.$ is asymptotically flat $\left|D \chi_{E}\right|$-a.e., but flatness should be understood only in the measuredGromov Hausdorff sense (for instance, a measure concentrated on a spiral would be asymptotically flat at the origin of the spiral). So, trying to get rectifiability using only this information is related to the search of rectifiability criteria where Jones' $\beta$ numbers are replaced by smaller numbers built with the scaled and localized Wasserstein distance. As far as we know, despite important progress on rectifiability criteria for measures (see for instance [T15, AT15]) this question is open even for measures in the Euclidean space.

Finally, for sets $E$ of finite perimeter in general $\operatorname{RCD}(K, N)$ m.m.s. $(X, \mathrm{~d}, \mathfrak{m})$, it would be important to get additional information on the structure of $\left|D \chi_{E}\right|$, besides the existence of flat tangents $\left|D \chi_{E}\right|$-a.e. in $X$.

Acknowledgements. The authors wish to thank Andrea Mondino for fruitful discussions around the topic of this note and the anonymous referee for several valuable comments that greatly improved the present note. 


\section{Preliminaries}

In a metric space $(Z, \mathrm{~d})$, we will denote by $B_{r}(x)=\{\mathrm{d}(\cdot, x)<r\}$ and $\bar{B}_{r}(x)=\{\mathrm{d}(\cdot, x) \leq r\}$ the open and closed balls respectively, by $\mathrm{C}_{\mathrm{bs}}(Z)$ the space of bounded continuous functions with bounded support, by $\operatorname{Lip}_{\mathrm{bs}}(Z) \subset \mathrm{C}_{\mathrm{bs}}(Z)$ the subspace of Lipschitz functions. We shall adopt the notation $\mathrm{C}_{\mathrm{b}}(Z)$ and $\operatorname{Lip}_{\mathrm{b}}(Z)$ for bounded continuous and bounded Lipschitz functions respectively. For any $f \in \operatorname{Lip}(Z)$ we shall denote by $\operatorname{Lip} f$ its global Lipschitz constant. The characteristic function with values in $\{0,1\}$ of a set $E \subset Z$ will be denoted by $\chi_{E}$.

The Borel $\sigma$-algebra of a metric space $(Z, \mathrm{~d})$ is denoted by $\mathscr{B}(Z)$. We shall denote by $\mathscr{M}(Z)$ the space of signed Borel measures with finite total variation on $Z$ and by $\mathscr{M}^{+}(Z), \mathscr{M}_{\text {loc }}^{+}(Z), \mathscr{P}(Z)$ the spaces of nonnegative finite Borel measures, nonnegative measures finite on bounded subsets of $Z$ and probability measures, respectively. We will denote by supp $\mathfrak{m}$ the support of any $\mathfrak{m} \in \mathscr{M}_{\text {loc }}^{+}(Z)$. We will use the standard notation $L^{p}(Z, \mathfrak{m}), L_{\mathrm{loc}}^{p}(Z, \mathfrak{m})$ for the $L^{p}$ spaces, whenever $\mathfrak{m}$ is nonnegative, and $\mathscr{L}^{n}, \mathcal{H}^{n}$ for the $n$-dimensional Lebesgue measure on $\mathbb{R}^{n}$ and the $n$-dimensional Hausdorff measure on a metric space, respectively. We shall denote by $\omega_{n}$ the Lebesgue measure of the unit ball in $\mathbb{R}^{n}$.

Below we list two useful lemmas. The proof of the first one, based on Cavalieri's formula, can be found for instance in [AGS15, Lemma 3.3] (notice that since we are assuming that $\mu$ and all $\mu_{n}$ are probability measures, weak convergence in duality w.r.t $\mathrm{C}_{\mathrm{bs}}(Z)$ and w.r.t. $\mathrm{C}_{\mathrm{b}}(Z)$ are equivalent).

Lemma 1.1. Let $\left(Z, \mathrm{~d}_{Z}\right)$ be a complete and separable metric space. Let $\left(\mu_{n}\right) \subset \mathscr{P}(Z)$ be weakly converging in duality with $\mathrm{C}_{\mathrm{bs}}(Z)$ to $\mu \in \mathscr{P}(Z)$ and let $f_{n}$ be uniformly bounded Borel functions such that

$$
\limsup _{n \rightarrow \infty} f_{n}\left(z_{n}\right) \leq f(z) \quad \text { whenever } \operatorname{supp} \mu_{n} \ni z_{n} \rightarrow z \in \operatorname{supp} \mu,
$$

for some Borel function $f$. Then

$$
\limsup _{n \rightarrow \infty} \int_{Z} f_{n} \mathrm{~d} \mu_{n} \leq \int_{Z} f \mathrm{~d} \mu .
$$

Remark 1.2. If $\left(Z, \mathrm{~d}_{Z}\right)$ is proper, $f_{n}$ and $f$ are continuous, and $\mu_{n}$ have uniformly bounded supports, then the uniform bound from above for $f_{n}$ is a direct consequence of (1.1).

The proof of Lemma 1.1 can be easily adapted to the case when we need to estimate the liminf of $\int_{Z} f_{n} \mathrm{~d} \mu_{n}$.

Lemma 1.3. Let $\left(Z, \mathrm{~d}_{Z}\right)$ be a complete and separable metric space. Let $\left(\mu_{n}\right)$ be a sequence of nonnegative Borel measures on $Z$ finite on bounded sets and assume that $\mu_{n}$ weakly converge to $\mu$ in duality w.r.t. $\mathrm{C}_{\mathrm{bs}}(Z)$. Let $\left(f_{n}\right)$ and $f$ be nonnegative Borel functions on $Z$ such that

$$
f(z) \leq \liminf _{n \rightarrow \infty} f_{n}\left(z_{n}\right) \quad \text { whenever } \operatorname{supp} \mu_{n} \ni z_{n} \rightarrow z \in \operatorname{supp} \mu .
$$

Then

$$
\int f \mathrm{~d} \mu \leq \liminf _{n \rightarrow \infty} \int f_{n} \mathrm{~d} \mu_{n}
$$

\subsection{Calculus tools}

Throughout this paper a metric measure space is a triple $(X, \mathrm{~d}, \mathfrak{m})$, where $(X, \mathrm{~d})$ is a locally compact and separable metric space (even though some intermediate results do not need the local compactness assumption) and $\mathfrak{m}$ is a nonnegative Borel measure on $X$ finite on bounded sets. We shall adopt the notation $(X, \mathrm{~d}, \mathfrak{m}, x)$ for pointed metric measure spaces, that is metric measure spaces $(X, \mathrm{~d}, \mathfrak{m})$ with a fixed reference point $x \in X$.

The Cheeger energy $\mathrm{Ch}: L^{2}(X, \mathfrak{m}) \rightarrow[0,+\infty]$ associated to a m.m.s. $(X, \mathrm{~d}, \mathfrak{m})$ is the convex and lower semicontinuous functional defined through

$$
\operatorname{Ch}(f):=\inf \left\{\liminf _{n \rightarrow \infty} \int \operatorname{lip}^{2} f_{n} \mathrm{dm}: \quad f_{n} \in \operatorname{Lip}_{\mathrm{b}}(X) \cap L^{2}(X, \mathfrak{m}),\left\|f_{n}-f\right\|_{2} \rightarrow 0\right\},
$$


where lip $f$ is the so called slope

$$
\operatorname{lip} f(x):=\limsup _{y \rightarrow x} \frac{|f(x)-f(y)|}{\mathrm{d}(x, y)} .
$$

The finiteness domain of the Cheeger energy will be denoted by $H^{1,2}(X, \mathrm{~d}, \mathfrak{m})$. We shall denote by $H_{\mathrm{loc}}^{1,2}(X, \mathrm{~d}, \mathfrak{m})$ the space of those functions $f$ such that $\eta f \in H^{1,2}(X, \mathrm{~d}, \mathfrak{m})$ for any $\eta \in \operatorname{Lip}_{\mathrm{bs}}(X, \mathrm{~d})$. Looking at the optimal approximating sequence in (1.3), it is possible to identify a canonical object $|\nabla f|$, called minimal relaxed slope, providing the integral representation

$$
\mathrm{Ch}(f):=\int_{X}|\nabla f|^{2} \mathrm{dm} \quad \forall f \in H^{1,2}(X, \mathrm{~d}, \mathfrak{m}) .
$$

Any metric measure space such that $\mathrm{Ch}$ is a quadratic form is said to be infinitesimally Hilbertian and from now on we shall always make this assumption, unless otherwise stated. Let us recall from [AGS14, G15] that, under this assumption, the function

$$
\nabla f_{1} \cdot \nabla f_{2}:=\lim _{\varepsilon \rightarrow 0} \frac{\left|\nabla\left(f_{1}+\varepsilon f_{2}\right)\right|^{2}-\left|\nabla f_{1}\right|^{2}}{2 \varepsilon}
$$

defines a symmetric bilinear form on $H^{1,2}(X, \mathrm{~d}, \mathfrak{m}) \times H^{1,2}(X, \mathrm{~d}, \mathfrak{m})$ with values into $L^{1}(X, \mathfrak{m})$.

It is possible to define a Laplacian operator $\Delta: \mathcal{D}(\Delta) \subset L^{2}(X, \mathfrak{m}) \rightarrow L^{2}(X, \mathfrak{m})$ in the following way. We let $\mathcal{D}(\Delta)$ be the set of those $f \in H^{1,2}(X, \mathrm{~d}, \mathfrak{m})$ such that, for some $h \in L^{2}(X, \mathfrak{m})$, one has

$$
\int_{X} \nabla f \cdot \nabla g \mathrm{~d} \mathfrak{m}=-\int_{X} h g \mathrm{~d} \mathfrak{m} \quad \forall g \in H^{1,2}(X, \mathrm{~d}, \mathfrak{m})
$$

and in that case we put $\Delta f=h$ since $h$ is uniquely determined by (1.4). It is easy to check that the definition is well-posed and that the Laplacian is linear (because $\mathrm{Ch}$ is a quadratic form).

The heat flow $P_{t}$ is defined as the $L^{2}(X, \mathfrak{m})$-gradient flow of $\frac{1}{2} \mathrm{Ch}$. Its existence and uniqueness follow from the Komura-Brezis theory. It can equivalently be characterized by saying that for any $u \in L^{2}(X, \mathfrak{m})$ the curve $t \mapsto P_{t} u \in L^{2}(X, \mathfrak{m})$ is locally absolutely continuous in $(0,+\infty)$ and satisfies

$$
\frac{\mathrm{d}}{\mathrm{d} t} P_{t} u=\Delta P_{t} u \quad \text { for } \mathscr{L}^{1} \text {-a.e. } t \in(0,+\infty), \quad \lim _{t \downarrow 0} P_{t} u=u \quad \text { in } L^{2}(X, \mathfrak{m}) .
$$

Under our assumptions the heat flow provides a linear, continuous and self-adjoint contraction semigroup in $L^{2}(X, \mathfrak{m})$. Moreover $P_{t}$ extends to a linear, continuous and mass preserving operator, still denoted by $P_{t}$, in all the $L^{p}$ spaces for $1 \leq p<+\infty$.

We recall the following regularization properties of $P_{t}$, ensured by the theory of gradient flows and maximal monotone operators (even without the infinitesimal Hilbertian assumption):

$$
\left\|P_{t} f\right\|_{L^{2}(X, \mathfrak{m})} \leq\|f\|_{L^{2}(X, \mathfrak{m})}, \quad \operatorname{Ch}\left(P_{t} f\right) \leq \frac{\|f\|_{L^{2}(X, \mathfrak{m})}^{2}}{2 t} \quad \text { and } \quad\left\|\Delta P_{t} f\right\|_{L^{2}(X, \mathfrak{m})} \leq \frac{\|f\|_{L^{2}(X, \mathfrak{m})}}{t}
$$

for any $t>0$ and for any $f \in L^{2}(X, \mathfrak{m})$.

Let us introduce now vector fields over $(X, \mathrm{~d}, \mathfrak{m})$ as derivations over an algebra of test functions, following the approach introduced in [We00] and adopted in [AT14] (see also [G13]).

Definition 1.4. We say that a linear functional $b: \operatorname{Lip}(X) \rightarrow L^{0}(X, \mathfrak{m})$ is a derivation if it satisfies the Leibniz rule, that is

$$
b(f g)=b(f) g+f b(g)
$$

for any $f, g \in \operatorname{Lip}(X)$.

Given a derivation $b$ and $p \in[1,+\infty]$, we write $b \in L^{p}(T X)$ (resp. $L_{\mathrm{loc}}^{p}(T X)$ ) if there exists $g \in L^{p}(X, \mathfrak{m})\left(\right.$ resp. $\left.L_{\mathrm{loc}}^{p}(X, \mathfrak{m})\right)$ such that

$$
|b(f)| \leq g \operatorname{lip}(f) \quad \mathfrak{m} \text {-a.e. on } X,
$$

for any $f \in \operatorname{Lip}(X)$ and we denote by $|b|$ the minimal (in the $\mathfrak{m}$-a.e. sense) $g$ with such property. We also say that $b$ has compact support if $|b|$ has compact support. 
Let us remark that any $f \in H^{1,2}(X, \mathrm{~d}, \mathfrak{m})$ defines in a canonical way a derivation $b_{f} \in L^{2}(T X)$ through the formula $b_{f}(g)=\nabla f \cdot \nabla g$, usually called the gradient derivation associated to $f$. We will use the notation $b \cdot \nabla f$ in place of $b(f)$ in the rest of the paper.

A notion of divergence can be introduced via integration by parts.

Definition 1.5. Let $b$ be a derivation in $L_{\text {loc }}^{1}(T X)$ and $p \in[1,+\infty]$. We say that $\operatorname{div} b \in L^{p}(X, \mathfrak{m})$ if there exists $g \in L^{p}(X, \mathfrak{m})$ such that

$$
\int_{X} b \cdot \nabla f \mathrm{~d} \mathfrak{m}=-\int_{X} g f \mathrm{~d} \mathfrak{m} \quad \text { for any } f \in \operatorname{Lip}_{\mathrm{bs}}(X) .
$$

By a density argument it is easy to check that such a $g$ is unique (when it exists) and we will denote it by $\operatorname{div} b$.

We refer to [G18] for the introduction of the so-called tangent and cotangent moduli over an arbitrary metric measure space and for the identification results between derivations in $L^{2}$ and elements of the tangent modulus $L^{2}(T X)$ which justify the use of this notation.

We conclude this brief subsection introducing the basic notions and results about functions of bounded variation and sets of finite perimeter over metric measure spaces. Let us remark that, for the sake of this discussion, the assumption that $\mathrm{Ch}$ is quadratic is unnecessary.

Definition 1.6 (Function of bounded variation). A function $f \in L^{1}(X, \mathfrak{m})$ is said to belong to the space $\operatorname{BV}(X, \mathrm{~d}, \mathfrak{m})$ if there exist locally Lipschitz functions $f_{i}$ converging to $f$ in $L^{1}(X, \mathfrak{m})$ such that

$$
\limsup _{i \rightarrow \infty} \int_{X}\left|\nabla f_{i}\right| \mathrm{d} \mathfrak{m}<+\infty \text {. }
$$

By localizing this construction one can define

$$
|D f|(A):=\inf \left\{\liminf _{i \rightarrow \infty} \int_{A}\left|\nabla f_{i}\right| \mathrm{dm}: f_{i} \in \operatorname{Lip}_{\mathrm{loc}}(A), \quad f_{i} \rightarrow f \text { in } L^{1}(A, \mathfrak{m})\right\}
$$

for any open $A \subset X$. In [ADM14] (see also [Mi03] for the case of locally compact spaces) it is proven that this set function is the restriction to open sets of a finite Borel measure that we call total variation of $f$ and still denote $|D f|$.

Dropping the global integrability condition on $f=\chi_{E}$, let us recall now the analogus definition of set of finite perimeter in a metric measure space (see again [A02, Mi03, ADM14]).

Definition 1.7 (Perimeter and sets of finite perimeter). Given a Borel set $E \subset X$ and an open set $A$ the perimeter $\operatorname{Per}(E, A)$ is defined in the following way:

$$
\operatorname{Per}(E, A):=\inf \left\{\liminf _{n \rightarrow \infty} \int_{A}\left|\nabla u_{n}\right| \mathrm{d} \mathfrak{m}: u_{n} \in \operatorname{Lip}_{\mathrm{loc}}(A), \quad u_{n} \rightarrow \chi_{E} \quad \text { in } L_{\mathrm{loc}}^{1}(A, \mathfrak{m})\right\} .
$$

We say that $E$ has finite perimeter if $\operatorname{Per}(E, X)<+\infty$. In that case it can be proved that the set function $A \mapsto \operatorname{Per}(E, A)$ is the restriction to open sets of a finite Borel measure $\operatorname{Per}(E, \cdot)$ defined by

$$
\operatorname{Per}(E, B):=\inf \{\operatorname{Per}(E, A): B \subset A, A \text { open }\} .
$$

Let us remark for the sake of clarity that $E \subset X$ with finite $\mathfrak{m}$-measure is a set of finite perimeter if and only if $\chi_{E} \in \operatorname{BV}(X, \mathrm{~d}, \mathfrak{m})$ and that $\operatorname{Per}(E, \cdot)=\left|D \chi_{E}\right|(\cdot)$. In the following we will say that $E \subset X$ is a set of locally finite perimeter if $\chi_{E}$ is a function of locally bounded variation, that is to say $\eta \chi_{E} \in \operatorname{BV}(X, \mathrm{~d}, \mathfrak{m})$ for any $\eta \in \operatorname{Lip}_{b s}(X, \mathrm{~d})$.

The following coarea formula for functions of bounded variation on metric measure spaces is taken from [Mi03, Proposition 4.2], dealing with locally compact spaces and its proof works in the more general setting of metric measure spaces. It will play a key role in the rest of the paper.

Theorem 1.8 (Coarea formula). Let $v \in \operatorname{BV}(X, \mathrm{~d}, \mathfrak{m})$. Then, $\{v>r\}$ has finite perimeter for $\mathscr{L}^{1}$-a.e. $r \in \mathbb{R}$ and, for any Borel function $f: X \rightarrow[0,+\infty]$, it holds

$$
\int_{X} f \mathrm{~d}|D v|=\int_{-\infty}^{+\infty}\left(\int_{X} f \mathrm{~d} \operatorname{Per}(\{v>r\}, \cdot)\right) \mathrm{d} r .
$$


By applying the coarea formula to the distance function we obtain immediately that, given $x \in X$, the ball $B_{r}(x)$ has finite perimeter for $\mathscr{L}^{1}$-a.e. $r>0$, and in the sequel this fact will also be used in the quantitative form provided by (1.10). We also recall (see for instance [A01, A02]) that sets of locally finite perimeter are an algebra, more precisely $\operatorname{Per}(E, B)=\operatorname{Per}(X \backslash E, B)$ and

$$
\operatorname{Per}(E \cap F, B)+\operatorname{Per}(E \cup F, B)=\operatorname{Per}(E, B)+\operatorname{Per}(F, B) .
$$

We will need also the following localized version of the coarea formula, which is an easy consequence of [Mi03, Remark 4.3].

Corollary 1.9. Let $v \in \mathrm{BV}(X, \mathrm{~d}, \mathfrak{m})$ be continuous and nonnegative. Then, for any Borel function $f: X \rightarrow[0,+\infty]$, it holds

$$
\int_{\{s \leq v<t\}} f \mathrm{~d}|D v|=\int_{s}^{t}\left(\int_{X} f \mathrm{~d} \operatorname{Per}(\{v>r\}, \cdot)\right) \mathrm{d} r, \quad 0 \leq s<t<+\infty .
$$

\section{$1.2 \operatorname{RCD}(K, N)$ metric measure spaces}

The main object of our investigation in this note are $\operatorname{RCD}(K, N)$ metric measure spaces, that is infinitesimally Hilbertian spaces satisfying a lower Ricci curvature bound and an upper dimension bound in synthetic sense according to [S06a, S06b, LV09]. Before than passing to the description of the main properties of $\operatorname{RCD}(K, N)$ spaces that will be relevant for the sake of this note, let us briefly focus on the adimensional case.

The class of $\operatorname{RCD}(K, \infty)$ spaces was introduced in [AGS14] (see also [AGMR15] for the extension to the case of $\sigma$-finite reference measures) adding to the $\operatorname{CD}(K, \infty)$ condition, formulated in terms of $K$-convexity properties of the logarithmic entropy over the Wasserstein space $\left(\mathscr{P}_{2}, W_{2}\right)$, the infinitesimally Hilbertianity assumption. Under the $\operatorname{RCD}(K, \infty)$ condition it was proved that the dual heat semigroup $P_{t}^{*}: \mathscr{P}_{2}(X) \rightarrow \mathscr{P}_{2}(X)$, defined by

$$
\int_{X} f \mathrm{~d} P_{t}^{*} \mu=\int_{X} P_{t} f \mathrm{~d} \mu \quad \forall \mu \in \mathscr{P}_{2}(X), \quad \forall f \in \operatorname{Lip}_{\mathrm{bs}}(X),
$$

is $K$-contractive w.r.t. the $W_{2}$-distance and, for $t>0$, maps probability measures into probability measures absolutely continuous w.r.t. $\mathfrak{m}$. Then, for any $t>0$, it is possible to define the heat kernel $p_{t}: X \times X \rightarrow[0,+\infty)$ by

$$
p_{t}(x, \cdot) \mathfrak{m}=P_{t}^{*} \delta_{x}
$$

We go on stating a few regularization properties of $\operatorname{RCD}(K, \infty)$ spaces, referring again to [AGS14, AGMR15] for a more detailed discussion and for the proofs of these results.

First we have the Bakry-Émery contraction estimate:

$$
\left|\nabla P_{t} f\right|^{2} \leq e^{-2 K t} P_{t}|\nabla f|^{2} \quad \mathfrak{m} \text {-a.e., }
$$

for any $t>0$ and for any $f \in H^{1,2}(X, \mathrm{~d}, \mathfrak{m})$. This contraction estimate can be generalized to the whole range of exponents $1<p<+\infty$, but in this note we will mainly be concerned with the case $p=1$. In [GH16] it has been proved that on any proper $\operatorname{RCD}(K, \infty)$ m.m.s. it holds

$$
\left|D P_{t} f\right| \leq e^{-K t} P_{t}^{*}|D f|,
$$

for any $t>0$ and for any $f \in \operatorname{BV}(X, \mathrm{~d}, \mathfrak{m})$.

Next we have the so called Sobolev to Lipschitz property, stating that any $f \in H^{1,2}(X, \mathrm{~d}, \mathfrak{m})$ such that $|\nabla f| \in L^{\infty}(X, \mathfrak{m})$ admits a representative $\tilde{f}$ such that $\operatorname{Lip}(\tilde{f}) \leq\||\nabla f|\|_{L^{\infty}}$, and the $L^{\infty}-\operatorname{Lip}$ regularization: for any $f \in L^{\infty}(X, \mathfrak{m})$ and $t>0$ one has $P_{t} f \in \operatorname{Lip}(X)$ with the quantitative estimate

$$
\sqrt{2 I_{2 K}(t)} \operatorname{Lip}\left(P_{t} f\right) \leq\|f\|_{L^{\infty}}
$$

where $I_{2 K}(t):=\int_{0}^{t} e^{2 K r} \mathrm{~d} r$.

Eventually let us introduce the space of test functions Test $(X, \mathrm{~d}, \mathfrak{m})$ following [G18]:

$$
\operatorname{Test}(X, \mathrm{~d}, \mathfrak{m}):=\left\{f \in D(\Delta) \cap L^{\infty}(X, \mathfrak{m}):|\nabla f| \in L^{\infty}(X, \mathfrak{m}) \quad \text { and } \quad \Delta f \in H^{1,2}(X, \mathrm{~d}, \mathfrak{m})\right\}
$$


We shall denote in the sequel by $\operatorname{Test}_{c}(X, \mathrm{~d}, \mathfrak{m})$ the space of test functions with compact support.

In the context of $\operatorname{RCD}(K, \infty)$ spaces it is possible to introduce a notion of flow associated to a vector field which reads as follows (see [AT14]).

Definition 1.10. Let us fix a vector field $b$. We say that a Borel map $\boldsymbol{X}:[0, \infty) \times X \rightarrow X$ is a Regular Lagrangian flow (RLF for short) associated to $b$ if the following conditions hold true:

1) $\boldsymbol{X}(0, x)=x$ and $X(\cdot, x) \in C([0, \infty) ; X)$ for every $x \in X$;

2) there exists $L \geq 0$, called compressibility constant, such that

$$
\boldsymbol{X}(t, \cdot)_{\sharp} \mathfrak{m} \leq L \mathfrak{m}, \quad \text { for every } t \geq 0 ;
$$

3) for every $f \in \operatorname{Test}(X, \mathrm{~d}, \mathfrak{m})$ the map $t \mapsto f(\boldsymbol{X}(t, x))$ is locally absolutely continuous in $[0, \infty)$ for $\mathfrak{m}$-a.e. $x \in X$ and

$$
\frac{\mathrm{d}}{\mathrm{d} t} f(\boldsymbol{X}(t, x))=b \cdot \nabla f(\boldsymbol{X}(t, x)) \quad \text { for a.e. } t \in(0, \infty) .
$$

The selection of "good" trajectories is encoded in condition 2), which is added to ensure that the RLF does not concentrate too much the reference measure $\mathfrak{m}$. We remark that the notion of RLF is stable under modification in a negligible set of initial conditions, but we prefer to work with a pointwise defined map in order to avoid technical issues.

It is well known that to obtain an existence and uniqueness theory for regular Lagrangian flows it is necessary to restrict to a class of sufficiently regular vector fields, even in the case of a smooth ambient space.

Below we introduce our working definition of Sobolev vector field with symmetric covariant derivative in $L^{2}$, following [AT14]. This definition is sufficient for our purposes, and weaker than the notion introduced in [G18], which corresponds to a sort of localized version of (1.18) (actually, we have been unable to prove differentiability in Gigli's stronger sense of our vector field $\left.b_{s}=\nabla P_{s} f /\left|\nabla P_{s} f\right|\right)$.

Definition 1.11. Let $b \in L^{\infty}(T X)$ with $\operatorname{div} b \in L^{\infty}(X, \mathfrak{m})$. We write $\left|\nabla_{\mathrm{sym}} b\right| \in L^{2}(X, \mathfrak{m})$ if there exists a constant $c>0$ such that

$$
\left|\int_{X} \nabla_{\mathrm{sym}} b(\nabla \varphi, \nabla \psi) \mathrm{d} \mathfrak{m}\right| \leq c\|\nabla \varphi\|_{L^{4}}\|\nabla \psi\|_{L^{4}} \quad \forall \varphi, \psi \in \operatorname{Test}(X, \mathrm{~d}, \mathfrak{m}),
$$

where

$$
\int_{X} \nabla_{\mathrm{sym}} b(\nabla \varphi, \nabla \psi) \mathrm{d} \mathfrak{m}:=-\frac{1}{2} \int_{X}\{b \cdot \nabla \varphi \Delta \psi+b \cdot \nabla \psi \Delta \varphi-\operatorname{div} b \nabla \varphi \cdot \nabla \psi\} \mathrm{d} \mathfrak{m} .
$$

We let $\left\|\nabla_{\mathrm{sym}} b\right\|_{L^{2}}$ be the smallest $c$ in (1.18). In particular we write $\nabla_{\mathrm{sym}} b=0$ if $\left\|\nabla_{\mathrm{sym}} b\right\|_{L^{2}}=0$.

In the next theorem we resume some general result concerning Regular Lagrangian flows that will be used in the sequel.

Theorem 1.12. Let $(X, \mathrm{~d}, \mathfrak{m})$ be an $\operatorname{RCD}(K, \infty)$ space for some $K \in \mathbb{R}$. Fix $b \in L^{\infty}(T X)$ with $\operatorname{div} b \in L^{\infty}(X, \mathfrak{m})$ and $\nabla_{\mathrm{sym}} b \in L^{2}(X, \mathfrak{m})$. Then

(i) there exists a unique regular Lagrangian flow $\boldsymbol{X}: \mathbb{R} \times X \rightarrow X$ associated to $b^{1}$ (uniqueness is understood in the following sense: if $\boldsymbol{X}$ and $\overline{\boldsymbol{X}}$ are Regular Lagrangian flows associated to $b$, then for $\mathfrak{m}$-a.e. $x \in X$ one has $\boldsymbol{X}_{t}(x)=\overline{\boldsymbol{X}}_{t}(x)$ for any $\left.t \in \mathbb{R}\right)$;

(ii) $\boldsymbol{X}$ satisfies the semigroup property: for any $s \in \mathbb{R}$ it holds that, for $\mathfrak{m}$-a.e. $x \in X$,

$$
\boldsymbol{X}(t, \boldsymbol{X}(s, x))=\boldsymbol{X}(t+s, x) \quad \forall t \in \mathbb{R},
$$

and the bound

$$
e^{-t\|\operatorname{div} b\|_{L} \infty} \mathfrak{m} \leq\left(\boldsymbol{X}_{t}\right)_{\sharp} \mathfrak{m} \leq e^{t\|\operatorname{div} b\|_{L} \infty} \mathfrak{m} ;
$$

\footnotetext{
${ }^{1}$ To be more precise, there exist unique Regular Lagrangian flows $\boldsymbol{X}^{+}, \boldsymbol{X}^{-}:[0,+\infty) \times X \rightarrow X$ associated to $b$ and $-b$ respectively and we let $\boldsymbol{X}_{t}=\boldsymbol{X}_{t}^{+}$for $t \geq 0$ and $\boldsymbol{X}_{t}=\boldsymbol{X}_{-t}^{-}$for $t \leq 0$.
} 
(iii) For any $\bar{u} \in L^{1}(X, \mathfrak{m}) \cap L^{\infty}(X, \mathfrak{m})$ there exists $u \in L_{\mathrm{loc}}^{\infty}\left(\mathbb{R} ; L^{1}(X, \mathfrak{m}) \cap L^{\infty}(X, \mathfrak{m})\right)$ such that $\left(\boldsymbol{X}_{t}\right)_{\sharp}(u \mathfrak{m})=u_{t} \mathfrak{m}$ and it solves the continuity equation, i.e. for any $\varphi \in \operatorname{Test}(X, \mathrm{~d}, \mathfrak{m})$ the map $t \mapsto \int_{X} \varphi u_{t} \mathrm{dm}$ is locally absolutely continuous with distributional derivative

$$
\frac{\mathrm{d}}{\mathrm{d} t} \int_{X} \varphi u_{t} \mathrm{~d} \mathfrak{m}=\int_{X}(b \cdot \nabla \varphi) u_{t} \mathrm{~d} \mathfrak{m}
$$

(iv) if $\operatorname{div} b=0$ and $\nabla_{\mathrm{sym}} b=0$ then $\boldsymbol{X}_{t}$ admits a representative which is a measure-preserving isometry, i.e.

$$
\mathrm{d}\left(\boldsymbol{X}_{t}(x), \boldsymbol{X}_{t}(y)\right)=\mathrm{d}(x, y) \quad \forall x, y \in X \quad \text { and } \quad\left(\boldsymbol{X}_{t}\right)_{\sharp} \mathfrak{m}=\mathfrak{m},
$$

for any $t \in \mathbb{R}$. Furthermore in this case the semigroup property (1.19) is satisfied pointwise.

Proof. (i), (ii), (iii) immediately follow from the results in [AT14] (see Theorem 8.3 together with Theorem 4.3 and Theorem 4.4). Let us prove (iv). From (1.20) we conclude that $\left(X_{t}\right)_{\sharp} \mathfrak{m}=\mathfrak{m}$ for any $t \in \mathbb{R}$. Let us now take $\bar{u} \in L^{\infty}(X, \mathfrak{m}) \cap W^{1,2}(X, \mathrm{~d}, \mathfrak{m})$ and $u$ as in (ii). Thanks to [AT14, Lemma 5.8] we get that $P_{\alpha} u_{t} \in \operatorname{Test}(X, \mathrm{~d}, \mathfrak{m})$ is still a solution of the continuity equation for any $\alpha \in(0,1)$. Then we can compute

$$
\begin{aligned}
\frac{\mathrm{d}}{\mathrm{d} t} \frac{1}{2} \int_{X}\left|\nabla P_{\alpha} u_{t}\right|^{2} \mathrm{~d} \mathfrak{m} & =-\frac{\mathrm{d}}{\mathrm{d} t} \frac{1}{2} \int_{X} P_{\alpha} u_{t} \Delta P_{\alpha} u_{t} \mathrm{~d} \mathfrak{m} \\
& =-\int_{X} b \cdot \nabla \Delta P_{\alpha} u_{t} P_{\alpha} u_{t} \mathrm{~d} \mathfrak{m} .
\end{aligned}
$$

Since $\operatorname{div} b=0$ and $\nabla_{\mathrm{sym}} b=0$, we deduce

$$
-\int_{X} b \cdot \nabla \Delta P_{\alpha} u_{t} P_{\alpha} u_{t} \mathrm{~d} \mathfrak{m}=\int_{X} b \cdot \nabla P_{\alpha} u_{t} P_{\alpha} \Delta u_{t} \mathrm{~d} \mathfrak{m}=0
$$

therefore

$$
\int_{X}\left|\nabla P_{\alpha} u_{t}\right|^{2} \mathrm{~d} \mathfrak{m}=\int_{X}\left|\nabla P_{\alpha} \bar{u}\right|^{2} \mathrm{~d} \mathfrak{m} \quad \forall t \in \mathbb{R}, \quad \forall \alpha \in(0,1) .
$$

Taking the limit in (1.21) as $\alpha \rightarrow 0$ it easily follows that $u_{t} \in H^{1,2}(X, \mathrm{~d}, \mathfrak{m})$ for any $t \in \mathbb{R}$ and that $\int_{X}\left|\nabla u_{t}\right|^{2} \mathrm{dm}$ does not depend on $t \in \mathbb{R}$. Using the identity $u_{t}(x)=\bar{u}(\boldsymbol{X}(-t, x)$ ) (which can be checked using the semigroup property (1.19) and $\left.\left(\boldsymbol{X}_{t}\right)_{\sharp} \mathfrak{m}=\mathfrak{m}\right)$ we deduce that, for any $t \in \mathbb{R}$,

$$
\operatorname{Ch}\left(\bar{u} \circ \boldsymbol{X}_{t}\right)=\operatorname{Ch}(\bar{u}) \quad \forall \bar{u} \in L^{\infty}(X, \mathfrak{m}) \cap H^{1,2}(X, \mathrm{~d}, \mathfrak{m}),
$$

and (iv) follows from arguments that have been used several times in the literature, as in [G13, Proposition 4.20].

As we anticipated above, $\operatorname{RCD}(K, N)$ spaces were introduced in [G15] as a finite dimensional counterpart of $\operatorname{RCD}(K, \infty)$. Here we just recall that they can be characterized asking for the quadraticity of $\mathrm{Ch}$, the volume growth condition $\mathfrak{m}\left(B_{r}(x)\right) \leq c_{1} \exp \left(c_{2} r^{2}\right)$ for some (and thus for all) $x \in X$, the validity of the Sobolev to Lipschitz property and of a weak form of Bochner's inequality

$$
\frac{1}{2} \Delta|\nabla f|^{2}-\nabla f \cdot \nabla \Delta f \geq \frac{(\Delta f)^{2}}{N}+K|\nabla f|^{2}
$$

for any $f \in \operatorname{Test}(X, \mathrm{~d}, \mathfrak{m})$. We refer to $[\operatorname{AMS} 15$, EKS15] for a more detailed discussion and equivalent characterizations of the $\operatorname{RCD}(K, N)$ condition.

Let us pass to a brief presentation of the main properties of $\operatorname{RCD}(K, N)$ spaces that will play a role in the sequel.

We recall that, as a consequence of the Bishop-Gromov inequality (see [V09, Theorem 30.11]), $\operatorname{RCD}(K, N)$ spaces are locally doubling, that is to say, for any $R>0$ there exists a constant $C_{D}$ depending only on $R, K$ and $N$ such that

$$
\mathfrak{m}\left(B_{2 r}(x)\right) \leq C_{D} \mathfrak{m}\left(B_{r}(x)\right),
$$


for any $x \in X$ and for any $0<r \leq R$. Another consequence of the Bishop-Gromov inequality is that $\mathfrak{m}\left(\partial B_{r}(x)\right)=0$ for any $x \in X$ and for any $r>0$.

In [G13] Gigli proved that in $\operatorname{RCD}(0, N)$ spaces the splitting theorem still holds, extending to this abstract framework the results obtained by Cheeger-Gromoll and Cheeger-Colding for smooth Riemannian manifolds and Ricci limit spaces, respectively.

Theorem 1.13. Let $(X, \mathrm{~d}, \mathfrak{m})$ be an $\operatorname{RCD}(0, N)$ m.m.s. containing a line, that is to say a curve $\gamma: \mathbb{R} \rightarrow X$ such that

$$
\mathrm{d}(\gamma(s), \gamma(t))=|t-s|, \quad \forall s, t \in \mathbb{R} .
$$

Then there exists a m.m.s. $\left(X^{\prime}, \mathrm{d}^{\prime}, \mathfrak{m}^{\prime}\right)$ such that $(X, \mathrm{~d}, \mathfrak{m})$ is isomorphic as a m.m.s. to

$$
\left(X^{\prime}, \mathrm{d}^{\prime}, \mathfrak{m}^{\prime}\right) \times\left(\mathbb{R}, \mathrm{d}_{\text {eucl }}, \mathscr{L}^{1}\right) .
$$

Furthermore:

(i) If $n \geq$ then $\left(X^{\prime}, \mathrm{d}^{\prime}, \mathfrak{m}^{\prime}\right)$ is an $\operatorname{RCD}(0, N-1)$;

(ii) if $N \in[1,2)$ then $X^{\prime}$ is a point.

Moreover, $\gamma(t)=\left(x^{\prime}, t\right)$ for any $t \in \mathbb{R}$, for some $x^{\prime} \in X^{\prime}$.

Since $\operatorname{RCD}(K, N)$ spaces are locally doubling and they satisfy a local Poincaré inequality (see [VR08]), the general theory of Dirichlet forms as developed in [S96] grants that we can find a locally Hölder continuous representative of the heat kernel $p$ on $X \times X \times(0,+\infty)$.

Moreover in [JLZ14] the following finer properties of the heat kernel over $\operatorname{RCD}(K, N)$ spaces have been proved: there exist constants $C=C_{K, N}>1$ and $c=c_{K, N} \geq 0$ such that

$$
\frac{1}{C \mathfrak{m}\left(B_{\sqrt{t}}(x)\right)} \exp \left\{-\frac{\mathrm{d}^{2}(x, y)}{3 t}-c t\right\} \leq p_{t}(x, y) \leq \frac{C}{\mathfrak{m}\left(B_{\sqrt{t}}(x)\right)} \exp \left\{-\frac{\mathrm{d}^{2}(x, y)}{5 t}+c t\right\}
$$

for any $x, y \in X$ and for any $t>0$. Moreover it holds

$$
\left|\nabla p_{t}(x, \cdot)\right|(y) \leq \frac{C}{\sqrt{t} \mathfrak{m}\left(B_{\sqrt{t}}(x)\right)} \exp \left\{-\frac{\mathrm{d}^{2}(x, y)}{5 t}+c t\right\} \quad \text { for } \mathfrak{m} \text {-a.e. } y \in X
$$

for any $t>0$ and for any $x \in X$. We remark that, in the case $K=0$, it is possible to take $c=0$.

Next we recall the notions of tangent space at a given point of a m.m.s. and of regular $k$ dimensional set. Given a m.m.s. $(X, \mathrm{~d}, \mathfrak{m}), x \in \operatorname{supp} \mathfrak{m}$ and $r \in(0,1)$ we shall consider the rescaled p.m.m.s. $\left(X, r^{-1} \mathrm{~d}, \mathfrak{m}_{r}^{x}, x\right)$, where

$$
\mathfrak{m}_{r}^{x}:=C(x, r)^{-1} \mathfrak{m}, \quad C(x, r):=\int_{B_{r}(x)}\left(1-\frac{1}{r} \mathrm{~d}(\cdot, x)\right) \mathrm{d} \mathfrak{m} .
$$

Definition 1.14. Let $(X, \mathrm{~d}, \mathfrak{m})$ be a m.m.s. and let $x \in \operatorname{supp} \mathfrak{m}$. We say that a p.m.m.s. $(Y, \varrho, \mu, y)$ is tangent to $(X, \mathrm{~d}, \mathfrak{m})$ if there exist $r_{i} \downarrow 0$ such that $\left(X, r_{i}^{-1} \mathrm{~d}, \mathfrak{m}_{r_{i}}^{x}, x\right)$ converge to $(Y, \varrho, \mu, y)$ in the pointed measured Gromov-Hausdorff topology (we refer to the forthcoming Definition 1.20 for the notion of pointed measured Gromov-Hausdorff convergence).

We shall denote by $\operatorname{Tan}_{x}(X, \mathrm{~d}, \mathfrak{m})$ the collection of all the tangent spaces of $(X, \mathrm{~d}, \mathfrak{m})$ at $x$.

Definition 1.15. Given an $\operatorname{RCD}(K, N)$ m.m.s. $(X, \mathrm{~d}, \mathfrak{m})$ we will say that $x \in X$ is a $k$-regular point for some integer $1 \leq k \leq N$ if $\operatorname{Tan}_{x}(X, \mathrm{~d}, \mathfrak{m})=\left\{\left(\mathbb{R}^{k}, \mathrm{~d}_{\text {eucl }}, c_{k} \mathscr{L}^{k}, 0^{k}\right)\right\}$, where

$$
c_{k}:=\left(\int_{B_{1}(0)}(1-|x|) \mathrm{d} x\right)^{-1}
$$

We shall denote by $\mathcal{R}_{k} \subset X$ the set of $k$-regular points of $(X, \mathrm{~d}, \mathfrak{m})$.

The following theorem sharpens one of the conclusions of [MN14] and has been proved in [BS18]. 
Theorem 1.16. Let $(X, \mathrm{~d}, \mathfrak{m})$ be an $\operatorname{RCD}(K, N)$ m.m.s.. Then there exists a unique integer $1 \leq k \leq N$ such that

$$
\mathfrak{m}\left(X \backslash \mathcal{R}_{k}\right)=0 .
$$

Let us conclude this subsection recalling the notion of non collapsed $\operatorname{RCD}(K, N)$ m.m.s., as introduced in [DPG18], and some useful property of this class.

Definition 1.17. Let $K \in \mathbb{R}$ and $N \geq 1$. We say that $(X, \mathrm{~d}, \mathfrak{m})$ is a non collapsed $\operatorname{RCD}(K, N)$ space, $\operatorname{ncRCD}(K, N)$ space for short, if it is $\operatorname{RCD}(K, N)$ and $\mathfrak{m}=\mathcal{H}^{N}$.

It is easy to check that, if $(X, \mathrm{~d}, \mathfrak{m})$ is $\operatorname{ncRCD}(K, N)$, then $N$ has to be an integer.

Below we state a useful regularity property of ncRCD spaces. Its validity follows from the volume cone-metric cone property (see [DPG16]) and the volume rigidity theorem (see [DPG18, Theorem 1.5]) with arguments analogous to the ones adopted in theory of non collapsed Ricci-limit spaces.

Theorem 1.18. Let $(X, \mathrm{~d}, \mathfrak{m})$ be a $\operatorname{ncRCD}(K, N)$ m.m.s. Assume that for some $x \in X$ it holds

$$
\left(\mathbb{R}^{N}, \mathrm{~d}_{\text {eucl }}, c_{N} \mathscr{L}^{N}, 0^{N}\right) \in \operatorname{Tan}_{x}(X, \mathrm{~d}, \mathfrak{m}) .
$$

Then $x$ is a regular point, that is to say the tangent at $x$ is unique (and $N$-dimensional Euclidean).

Proof. The conclusion follows from [DPG18, Proposition 2.10], we just provide here a sketch of the proof for sake of completeness. Let $r_{i} \downarrow 0$ be a sequence of scales such that the rescalings of the p.m.m.s. $(X, \mathrm{~d}, \mathfrak{m}, x)$ converge in the pmGH topology to $\left(\mathbb{R}^{N}, \mathrm{~d}_{\text {eucl }}, c_{N} \mathscr{L}^{N}, 0^{N}\right)$ as $i \rightarrow \infty$. Since $\mathscr{L}^{N}\left(\partial B_{1}(0)\right)=0$, we get

$$
\lim _{i \rightarrow \infty} \mathfrak{m}\left(B_{r_{i}}(x)\right) /\left(c_{N} \omega_{N} r_{i}^{N}\right)=1
$$

The Bishop-Gromov inequality allows then to improve this conclusion to

$$
\lim _{r \rightarrow 0} \mathfrak{m}\left(B_{r}(x)\right) /\left(c_{N} \omega_{N} r^{N}\right)=1,
$$

thus, for any $\left(Y, \mathrm{~d}_{Y}, \mathfrak{m}_{Y}, y\right) \in \operatorname{Tan}_{x}(X, \mathrm{~d}, \mathfrak{m})$ we have that $\mathfrak{m}_{Y}=c_{N} \mathcal{H}^{N}$ by [DPG18, Theorem 1.2] and $\mathcal{H}^{N}\left(B_{1}^{Y}(y)\right)=\omega_{N}$. Hence the volume rigidity theorem [DPG18, Theorem 1.5] applies, yielding that $B_{1 / 2}^{Y}(y)$ is isometric to $B_{1 / 2}^{\mathbb{R}^{N}}(0)$. Eventually, thanks to the fact that $\left(Y, \mathrm{~d}_{Y}, \mathfrak{m}_{Y}, y\right)$ is a metric cone with tip $y$, we conclude that it is isomorphic to $\mathbb{R}^{N}$.

Remark 1.19. Let us remark that there is no analogue of Theorem 1.18 without the non collapsing assumption. Indeed Menguy built in [Me01] an example of Ricci limit space with a strictly weakly regular point, that is to say a point with an Euclidean space in the tangent cone whose tangent cone is not unique.

\subsection{Convergence and stability results for sequences of $\operatorname{RCD}(K, N)$ spaces}

We dedicate this subsection to an overview of the subject of convergence and stability for Sobolev functions defined on converging sequences of metric measure spaces. The main references for this part are [GMS15] and [AH17].

Definition 1.20. A sequence $\left\{\left(X_{i}, \mathrm{~d}_{i}, \mathfrak{m}_{i}, x_{i}\right)\right\}_{i \in \mathbb{N}}$ of pointed m.m.s. is said to converge in the pmGH topology to $(Y, \varrho, \mu, y)$ if there exist a complete separable metric space $\left(Z, \mathrm{~d}_{Z}\right)$ and isometric embeddings

$$
\begin{aligned}
& \Psi_{i}:\left(\operatorname{supp} \mathfrak{m}_{i}, \mathrm{~d}_{i}\right) \rightarrow\left(Z, \mathrm{~d}_{Z}\right) \quad \forall i \in \mathbb{N}, \\
& \Psi:(\operatorname{supp} \mu, \varrho) \rightarrow\left(Z, \mathrm{~d}_{Z}\right),
\end{aligned}
$$

such that for every $\varepsilon>0$ and $R>0$ there exists $i_{0}$ such that for every $i>i_{0}$

$$
\Psi\left(B_{R}^{Y}(y)\right) \subset\left[\Psi_{i}\left(B_{R}^{X_{i}}\left(x_{i}\right)\right)\right]_{\varepsilon}, \quad \Psi_{i}\left(B_{R}^{X_{i}}\left(x_{i}\right)\right) \subset\left[\Psi\left(B_{R}^{Y}(y)\right)\right]_{\varepsilon},
$$

where $[A]_{\varepsilon}:=\left\{z \in Z: \mathrm{d}_{Z}(z, A)<\varepsilon\right\}$ for every $A \subset Z$. Moreover $\left(\Psi_{i}\right)_{\#} \mathfrak{m}_{i} \rightarrow \Psi_{\#} \mu$, where the convergence is understood in duality with $\mathrm{C}_{\mathrm{bs}}(Z)$. 
In the case of a sequence of uniformly locally doubling metric measure spaces $\left(X_{i}, \mathrm{~d}_{i}, \mathfrak{m}_{i}, x_{i}\right)$ (as in the case of $\operatorname{RCD}(K, N)$ spaces), the pointed measured Gromov-Hausdorff convergence to $(Y, \varrho, \mu, y)$ can be equivalently characterized asking for the existence of a proper metric space $\left(Z, \mathrm{~d}_{Z}\right)$ such that all the metric spaces $\left(X_{i}, \mathrm{~d}_{i}\right)$ are isometrically embedded into $\left(Z, \mathrm{~d}_{Z}\right), x_{i} \rightarrow y$ and $\mathfrak{m}_{i} \rightarrow \mu$ in duality with $\mathrm{C}_{\mathrm{bs}}(Z)$. This is the so called extrinsic approach, that we shall adopt in the rest of the note.

Definition 1.21. Let $\left(X_{i}, \mathrm{~d}_{i}, \mathfrak{m}_{i}, x_{i}\right)$ be pointed m.m.s. converging in the pmGH topology to $(Y, \varrho, \mu, y)$ and let $f_{i}: X_{i} \rightarrow \mathbb{R}, f: Y \rightarrow \mathbb{R}$. Assume the convergence to be realized into a common metric space $\left(Z, \mathrm{~d}_{Z}\right)$ as above. Then we say that $f_{i} \rightarrow f$ pointwise if $f_{i}\left(x_{i}\right) \rightarrow f(x)$ for every sequence of points $x_{i} \in X_{i}$ such that $x_{i} \rightarrow x$ in $Z$. If moreover for every $\varepsilon>0$ there exists $\delta>0$ such that $\left|f_{i}\left(x_{i}\right)-f(x)\right| \leq \varepsilon$ for every $i \geq \delta^{-1}$ and every $x_{i} \in X_{i}, x \in Y$ with $\mathrm{d}_{Z}\left(x_{i}, x\right) \leq \delta$, then we say that $f_{i} \rightarrow f$ uniformly.

Remark 1.22. Let us point out that, if all the spaces coincide and are compact, then $f_{i} \rightarrow f$ pointwise according to Definition 1.21 if and only if $f$ is continuous and $f_{i} \rightarrow f$ uniformly. Therefore the terminology "pointwise convergence" might be a bit misleading. Nevertheless we prefer to keep using it since this terminology is used in several other works [AH17, AHT18, MN14].

We recall below the notions of convergence in $L^{p}$ and Sobolev spaces for functions defined over converging sequences of metric measure spaces. We will be concerned only with the cases $p=2$ and $p=1$ in the rest of the note. We refer again to [AH17, GMS15] for a more general treatment and the proofs of the results we state below.

Definition 1.23. We say that $f_{i} \in L^{2}\left(X_{i}, \mathfrak{m}_{i}\right)$ converge in $L^{2}$-weak to $f \in L^{2}(Y, \mu)$ if $f_{i} \mathfrak{m}_{i} \rightarrow f \mu$ in duality with $\mathrm{C}_{\mathrm{bs}}(Z)$ and $\sup _{i}\left\|f_{i}\right\|_{L^{2}\left(X_{i}, \mathrm{~m}_{i}\right)}<+\infty$.

We say that $f_{i} \in L^{2}\left(X_{i}, \mathfrak{m}_{i}\right)$ converge in $L^{2}$-strong to $f \in L^{2}(Y, \mu)$ if $f_{i} \mathfrak{m}_{i} \rightarrow f \mu$ in duality with $\mathrm{C}_{\mathrm{bs}}(Z)$ and $\lim _{i}\left\|f_{i}\right\|_{L^{2}\left(X_{i}, \mathfrak{m}_{i}\right)}=\|f\|_{L^{2}(Y, \mu)}$.

Definition 1.24. We say that $f_{i} \in H^{1,2}\left(X_{i}, \mathrm{~d}_{i}, \mathfrak{m}_{i}\right)$ are weakly convergent to $f \in H^{1,2}(Y, \varrho, \mu)$ if they converge in $L^{2}$-weak and $\sup _{i} \mathrm{Ch}^{i}\left(f_{i}\right)<+\infty$. Strong $H^{1,2}$-converge is defined asking that $f_{i}$ converge to $f$ in $L^{2}$-strong and $\lim _{i} \mathrm{Ch}^{i}\left(f_{i}\right)=\mathrm{Ch}(f)$.

From now until the end of this subsection we always assume that $\left(X_{i}, \mathrm{~d}_{i}, \mathfrak{m}_{i}\right)$ are $\operatorname{RCD}(K, N)$ metric measure spaces for any $i \in \mathbb{N}$.

The following localized lower semicontinuity result will play a role in the sequel of the note. It is taken from [AH17, Lemma 5.8].

Proposition 1.25. Let $f_{i} \in H^{1,2}\left(X_{i}, \mathrm{~d}_{i}, \mathfrak{m}_{i}\right)$ be weakly converging in $H^{1,2}$ to $f \in H^{1,2}(Y, \varrho, \mu)$. Then

$$
\liminf _{i \rightarrow \infty} \int_{Z} g\left|\nabla f_{i}\right| \mathrm{dm}_{i} \geq \int_{Z} g|\nabla f| \mathrm{d} \mu, \quad \text { for any nonnegative } g \in \operatorname{Lip}_{\mathrm{bs}}(Z) \text {. }
$$

Definition 1.26. We say that a sequence $\left(f_{i}\right) \subset L^{1}\left(X_{i}, \mathfrak{m}_{i}\right)$ converges $L^{1}$-strongly to $f \in L^{1}(Y, \mu)$ if

$$
\sigma \circ f_{i} \mathfrak{m}_{i} \rightarrow \sigma \circ f \mu \quad \text { and } \quad \int_{X_{i}}\left|f_{i}\right| \mathrm{d} \mathfrak{m}_{i} \rightarrow \int_{Y}|f| \mathrm{d} \mu
$$

where $\sigma(z):=\operatorname{sign}(z) \sqrt{|z|}$ and the weak convergence is understood in duality with $\mathrm{C}_{\mathrm{bs}}(Z)$. Equivalently, if $\sigma \circ f_{i} L^{2}$-strongly converge to $\sigma \circ f$.

We say that $f_{i} \in \mathrm{BV}\left(X_{i}, \mathfrak{m}_{i}\right)$ converge in energy in $\mathrm{BV}$ to $f \in \mathrm{BV}(Y, \mu)$ if $f_{i}$ converge $L^{1}$ strongly to $f$ and

$$
\lim _{i \rightarrow \infty}\left|D f_{i}\right|\left(X_{i}\right)=|D f|(Y) .
$$

Remark 1.27. The presence of the function $\sigma$ in the definition of $L^{1}$-strong convergence is necessary due to the lack of reflexivity of $L^{1}$. Indeed the counterpart of Definition 1.23 in the case $p=1$ is easily seen to be not equivalent to convergence in $L^{1}$ norm when all the spaces coincide.

The following useful stability result is part of [AH17, Proposition 3.3]. 
Proposition 1.28. Let $p \in\{1,2\}$. If $f_{i} \in L^{p}\left(X_{i}, \mathfrak{m}_{i}\right)$ converge in $L^{p}$-strong to $f \in L^{p}(Y, \mu)$ then $\varphi \circ f_{i}$ converge to $\varphi \circ f$ in $L^{p}$-strong for any $\varphi \in \operatorname{Lip}(\mathbb{R})$ such that $\varphi(0)=0$. In particular, if $g_{i}$ are uniformly bounded in $L^{\infty}$ and $L^{1}$-strongly convergent to $g$ then

$$
\lim _{i \rightarrow \infty}\left\|g_{i}\right\|_{L^{p}\left(X_{i}, \mathfrak{m}_{i}\right)}=\|g\|_{L^{p}(Y, \mu)} .
$$

If $f_{i}, g_{i} \in L^{p}\left(X_{i}, \mathfrak{m}_{i}\right)$ converge in $L^{p}$-strong to $f$ and $g$ respectively, then $f_{i}+g_{i}$ converge to $f+g$ in $L^{p}$-strong.

Below we quote a useful compactness criterion borrowed from [GMS15, Theorem 6.3] (see also [AH17, Theorem 7.4]).

Theorem 1.29. Let $f_{i} \in H^{1,2}\left(X_{i}, \mathrm{~d}_{i}, \mathfrak{m}_{i}\right)$ be such that

$$
\sup _{i}\left\{\int_{Z}\left|f_{i}\right|^{2} \mathrm{dm}_{i}+\operatorname{Ch}^{i}\left(f_{i}\right)\right\}<+\infty
$$

and

$$
\lim _{R \rightarrow \infty} \limsup _{i \rightarrow \infty} \int_{Z \backslash B_{R}(\bar{z})}\left|f_{i}\right|^{2} \mathrm{~d} \mathfrak{m}_{i}=0,
$$

for some (and thus for all) $\bar{z} \in Z$. Then $\left(f_{i}\right)$ has a $L^{2}$-strongly convergent subsequence to $f \in$ $H^{1,2}(Y, \varrho, \mu)$.

Next we pass to a stability/compactness criterion in $H^{1,2}$. Its statement is taken from [AH17, Corollary 5.5].

Proposition 1.30. (a) If $f_{i} \in H^{1,2}\left(X_{i}, \mathrm{~d}_{i}, \mathfrak{m}_{i}\right), f_{i} \in \mathcal{D}\left(\Delta_{i}\right)$ converge in $L^{2}$-strong to $f$ and $\Delta_{i} f_{i}$ are uniformly bounded in $L^{2}$, then $f \in \mathcal{D}(\Delta), \Delta_{i} f_{i}$ converge in $L^{2}$-weak to $\Delta f$ and $f_{i}$ converge in $H^{1,2}$-strong to $f$;

(b) for all $t>0, P_{t}^{i} f_{i}$ converge in $H^{1,2}$-strong to $P_{t} f$ whenever $f_{i}$ converge in $L^{2}$-strong to $f$.

We conclude this subsection with a localized convergence result taken from [AH17, Theorem $5.7]$.

Theorem 1.31. Let $v_{i}, w_{i} \in H^{1,2}\left(X_{i}, \mathrm{~d}_{i}, \mathfrak{m}_{i}\right)$ be strongly convergent in $H^{1,2}$ to $v, w \in H^{1,2}(Y, \varrho, \mu)$, respectively. Then $\nabla v_{i} \cdot \nabla w_{i}$ converge $L^{1}$-strongly to $\nabla v \cdot \nabla w$.

\section{Rigidity of the 1-Bakry-Émery inequality and splitting theorem}

Our aim in this section is to prove a rigidity result for $\operatorname{RCD}(0, N)$ spaces admitting a non constant function satisfying the equality in the Bakry-Émery inequality for exponent $p=1$ (1.14). Our investigation of the consequences of this rigidity property was motivated by the study of blow-ups of sets of finite perimeter (see Theorem 4.2 below).

Theorem 2.1. Let $(X, \mathrm{~d}, \mathfrak{m})$ be an $\operatorname{RCD}(0, N)$ m.m.s.. Assume that there exist a non constant function $f \in \operatorname{Lip}_{\mathrm{b}}(X)$ and $s>0$ satisfying

$$
\left|\nabla P_{s} f\right|=P_{s}|\nabla f| \quad \mathfrak{m} \text {-a.e. in } X .
$$

Then there exists a m.m.s. $\left(X^{\prime}, \mathrm{d}^{\prime}, \mathfrak{m}^{\prime}\right)$ such that $X$ is isomorphic, as a metric measure space, to $X^{\prime} \times \mathbb{R}$. Furthermore:

(i) if $N \geq 2$ then $\left(X^{\prime}, \mathrm{d}^{\prime}, \mathfrak{m}^{\prime}\right)$ is an $\operatorname{RCD}(0, N-1)$ m.m.s.;

(ii) if $N \in[1,2)$ then $X^{\prime}$ is a point. 
Moreover, the function $f$ written in coordinates $\left(x^{\prime}, t\right) \in X^{\prime} \times \mathbb{R}$ depends only on the variable $t$ and it is monotone.

Remark 2.2. Let us point out that the action of the heat semigroup in $L^{\infty}(X, \mathfrak{m})$ can be defined by mean of

$$
P_{t} f(x):=\int_{X} f(y) p_{t}(x, y) \mathrm{d} \mathfrak{m}(y),
$$

where $p_{t}$ is the heat kernel (see (1.12)). Using an approximation argument is it possible to see that, for any $f \in L^{\infty}(X, \mathfrak{m})$ and every $\varphi \in L^{1}(X, \mathfrak{m})$ the map $t \rightarrow \int_{X} P_{t} f \varphi \mathrm{dm}$ is absolutely continuous with derivative

$$
\frac{\mathrm{d}}{\mathrm{d} t} \int_{X} P_{t} f \varphi \mathrm{d} \mathfrak{m}=\int_{X} \Delta P_{t} f \varphi \mathrm{dm},
$$

in other words $P_{t} f$ is still a solution of the heat equation.

Remark 2.3. The assumption $f \in \operatorname{Lip}_{\mathrm{b}}(X)$ in Theorem 1.13 can be replaced with the more general $f \in \operatorname{Lip}(X)$, provided we extend the action of the heat semigroup to the class of Borel functions with at most linear growth at infinity, i.e.

$$
|f(x)| \leq C\left(1+\mathrm{d}\left(x, x_{0}\right)\right) \quad \text { for any } x \in X
$$

for some $x_{0} \in X$ and $C \geq 0$. Even though under the $\operatorname{RCD}(0, N)$ condition the Gaussian estimates for the heat kernel provide this extension, we shall consider only the case $f \in \operatorname{Lip}_{\mathrm{b}}(X)$ that is enough for our purposes.

In order to better motivate the statement of Theorem 2.1 let us spend a few words about the rigidity case in the Bakry Émery inequality for $p=2$. Assume that $\left(M^{n}, \mathrm{~d}_{g}, e^{-V} \operatorname{Vol}_{g}\right)$ is a smooth weighted Riemannian manifold with nonnegative generalized $N$-Ricci tensor $\operatorname{Ric}_{N}$, where

$$
\operatorname{Ric}_{N}:=\operatorname{Ric}+\operatorname{Hess} V-\frac{\nabla V \otimes \nabla V}{N-n},
$$

and the last term is defined to be 0 when $V$ is constant and $N=n$. Let $f: M \rightarrow \mathbb{R}$ be such that $\left|\nabla P_{t} f\right|^{2}=P_{t}|\nabla f|^{2}$ for some $t>0$. Then we can compute

$$
\begin{aligned}
0 & =P_{t}|\nabla f|^{2}-\left|\nabla P_{t} f\right|^{2}=\int_{0}^{t} \frac{\mathrm{d}}{\mathrm{d} s} P_{s}\left|\nabla P_{t-s} f\right|^{2} \mathrm{~d} s \\
& =2 \int_{0}^{t} P_{s}\left(\left|\operatorname{Hess} P_{t-s} f\right|^{2}+\operatorname{Ric}_{N}\left(\nabla P_{t-s} f, \nabla P_{t-s} f\right)+\frac{\left(\nabla V \cdot \nabla P_{t-s} f\right)^{2}}{N-n}\right) \mathrm{d} s,
\end{aligned}
$$

where the second equality follows from the generalized Bochner identity and $\Delta$ is the weighted Laplacian. Therefore Hess $f \equiv 0,(\nabla V \cdot \nabla f)^{2} \equiv 0$. Thus $\Delta f \equiv 0$ since

$$
\frac{(\Delta f)^{2}}{N} \leq|\operatorname{Hess} f|^{2}+\frac{(\nabla V \cdot \nabla f)^{2}}{N-n}=0 .
$$

Using a standard argument we obtain that $M^{n}$ splits isometrically as $L \times \mathbb{R}$ for some Riemannian manifold $L$. Taking into account the fact that $\Delta f=0$ we can prove that also the measure splits.

Furthermore, denoting by $z, t$ the coordinates on $L$ and $\mathbb{R}$ respectively, it holds that $P_{s} f(z, t)=$ $f(z, t)=\alpha t$ for any $s \geq 0$ and for any $t \in \mathbb{R}$, for some constant $\alpha \neq 0$.

Passing to the study of the case $p=1$, any function $f: \mathbb{R}^{n} \rightarrow \mathbb{R}$ such that $\left|\nabla P_{t} f\right| \equiv P_{t}|\nabla f|$ is of the form $f(z)=\phi(z \cdot v)$ for some monotone function $\phi: \mathbb{R} \rightarrow \mathbb{R}$ and some $v \in \mathbb{R}^{n}$. This is due to the commutation between gradient operator and heat flow on the Euclidean space and to the characterization of the equality case in Jensen's inequality. More in general, thanks to the tensorization property of the heat flow, it is possible to check that on any product m.m.s. $X=X^{\prime} \times \mathbb{R}$, any function $f$ depending only on the variable $t \in \mathbb{R}$ in a monotone way satisfies $\left|\nabla P_{t} f\right|=P_{t}|\nabla f|$ almost everywhere. Basically Theorem 2.1 is telling us that, in the setting of $\operatorname{RCD}(0, N)$ spaces, this is the only possible case.

About the strategy of the proof let us observe that, as the examples above illustrated show, in the rigidity case for $p=1$ it is not necessarily true that the rigid function has vanishing 
Hessian. Therefore we cannot directly use $P_{s} f$ as a splitting function. Still our strategy relies on the properties of the normalized gradient $\nabla P_{s} f /\left|\nabla P_{s} f\right|$. First we will prove that it has vanishing symmetric covariant derivative and then that its flow lines are metric lines. The conclusion will be eventually achieved building upon the splitting theorem.

Let us start proving that if the rigidity condition (2.1) holds for some $s>0$ then it must hold for any $s \geq 0$.

Lemma 2.4. Let $(X, \mathrm{~d}, \mathfrak{m})$ be an $\operatorname{RCD}(0, N)$ metric measure space and $f \in \operatorname{Lip}_{\mathrm{b}}(X)$. If there exists $s>0$ such that

$$
\left|\nabla P_{s} f\right|=P_{s}|\nabla f| \quad \mathfrak{m} \text {-a.e. in } X,
$$

Then $\left|\nabla P_{r} f\right|=P_{r}|\nabla f|$ for any $r \geq 0$.

Proof. It is simple to check that $\left|\nabla P_{r} f\right|=P_{r}|\nabla f|$ for any $0 \leq r \leq s$. Indeed, using (2.3) and the Bakry-Émery inequality (1.14), we have

$$
0 \leq P_{s-r}\left(P_{r}|\nabla f|-\left|\nabla P_{r} f\right|\right)=P_{s}|\nabla f|-P_{s-r}\left|\nabla P_{r} f\right|=\left|\nabla P_{s} f\right|-P_{s-r}\left|\nabla P_{r} f\right| \leq 0 .
$$

Let us now fix $\varphi \in \operatorname{Test}_{c}(X, \mathrm{~d}, \mathfrak{m})$ and set

$$
F(r):=\int_{X}\left(\left(P_{r}|\nabla f|\right)^{2}-\left|\nabla P_{r} f\right|^{2}\right) \varphi \mathrm{dm} .
$$

We claim that $F(r)$ is a real analytic function in $(0,+\infty)$. Observe that the claim, together with the information $F \equiv 0$ in $(0, s)$, implies $F(r)=0$ for any $r \geq 0$ and thus our conclusion, due to the arbitrariness of the test function.

Integrating by parts the right hand side in (2.4) and using (1.5), we can write

$$
F(r)=\int_{X}\left(P_{r}|\nabla f|\right)^{2} \varphi \mathrm{d} \mathfrak{m}+\frac{1}{2} \frac{\mathrm{d}}{\mathrm{d} r} \int_{X}\left(P_{r} f\right)^{2} \varphi \mathrm{d} \mathfrak{m}-\frac{1}{2} \int_{X}\left(P_{r} f\right)^{2} \Delta \varphi \mathrm{d} \mathfrak{m},
$$

so the claim is a consequence of Lemma 2.5 below.

Lemma 2.5. Let $(X, \mathrm{~d}, \mathfrak{m})$ be an $\operatorname{RCD}(K, N)$ m.m.s.. For any $g \in L^{\infty}(X, \mathfrak{m})$ and any $\varphi \in$ $L^{1}(X, \mathfrak{m})$ the map $t \mapsto \int_{X}\left(P_{t} g\right)^{2} \varphi \mathrm{d} \mathfrak{m}$ is real analytic in $(0,+\infty)$.

Proof. Exploiting a well-known analyticity criterion for real functions, it is enough to show, for any $[a, b] \subset(0,+\infty)$, the existence of a constant $C=C(K, N, a, b)$ such that

$$
\left|\frac{\mathrm{d}^{n}}{\mathrm{~d} t^{n}} \int_{X}\left(P_{t} g\right)^{2} \varphi \mathrm{dm}\right| \leq C^{n}\|g\|_{L^{\infty}}^{2}\|\varphi\|_{L^{1}} \quad \forall t \in(a, b), \quad \forall n \in \mathbb{N} .
$$

Observe that $(2.5)$ can be checked commuting the operators $P_{t}$ and $\Delta$ and using iteratively the estimate

$$
\left\|\Delta P_{t} g\right\|_{L^{\infty}} \leq C^{\prime}\|g\|_{L^{\infty}} \quad \forall t \in(a, b),
$$

where $C^{\prime}>0$ depends only on $N, K, a$ and $b$.

Let us prove (2.6) arguing by duality. For any $\psi \in L^{1} \cap L^{2}(X, \mathfrak{m})$, we have

$$
\begin{aligned}
\left|\int_{X} \Delta P_{t} g \psi \mathrm{d} \mathfrak{m}\right| & =\left|\int_{X} \nabla P_{t / 2} g \cdot \nabla P_{t / 2} \psi \mathrm{d} \mathfrak{m}\right| \\
& \leq\left\|\nabla P_{t / 2} g\right\|_{L^{\infty}}\left\|\nabla P_{t / 2} \psi\right\|_{L^{1}} \\
& \leq C^{\prime \prime}\|g\|_{L^{\infty}} C^{\prime \prime}\|\psi\|_{L^{1}},
\end{aligned}
$$

where the last inequality is a consequence of the following general fact: there exists $C^{\prime \prime}(N, K, a, b)>$ 0 such that

$$
\left\|\nabla P_{t} h\right\|_{L^{p}} \leq C^{\prime \prime}\|h\|_{L^{p}} \quad \forall t \in(a, b), \quad \forall h \in L^{p}(X, \mathfrak{m}) \text { with } 1 \leq p \leq \infty .
$$

In order to check (2.7) we use the Gaussian estimates for the heat kernel and its gradient (1.22), (1.23) obtaining that there exists a constant $\alpha>1$ such that

$$
\left|\nabla P_{t} h\right|(x) \leq C^{\prime \prime} P_{\alpha t}|h|(x), \quad \text { for m-a.e. } x \in X, \quad \forall t \in(a, b),
$$

and we take the $L^{p}$ norm both sides. 
Let us introduce the most important object of our investigation. For any $s>0$ we consider the vector field

$$
b_{s}:=\frac{\nabla P_{s} f}{P_{s}|\nabla f|}
$$

that, since $P_{s}|\nabla f|>0 \mathfrak{m}$-a.e., is well defined and satisfies

$$
\left|b_{s}\right|=1 \quad \mathfrak{m} \text {-a.e. in } X, \quad \forall s>0,
$$

thanks to (2.1).

The first important ingredient of the proof of Theorem 2.1 is the following proposition. Its proof is inspired by an analogous result in [G13].

Proposition 2.6 (Variation formula, version 1). For any $s>0, t \geq 0$ and any $g \in \operatorname{Test}(X, \mathrm{~d}, \mathfrak{m})$ it holds

$$
b_{t+s} \cdot \nabla P_{t} g=P_{t}\left(b_{s} \cdot \nabla g\right), \quad \mathfrak{m} \text {-a.e. in } X \text {. }
$$

Before proving Proposition 2.6 we need to state a simple lemma.

Lemma 2.7. For any $s \geq 0$ the function $P_{s} f$ satisfies

$$
\left|\nabla P_{t+s} f\right|=P_{t}\left|\nabla P_{s} f\right|, \quad \mathfrak{m} \text {-a.e. in } X, \quad \forall t \geq 0 \text {. }
$$

Proof. Using first the Bakry-Émery inequality (1.14) and then twice (2.1) we get

$$
\left|\nabla P_{t+s} f\right| \leq P_{t}\left|\nabla P_{s} f\right|=P_{t+s}|\nabla f|=\left|\nabla P_{t+s} f\right|
$$

that proves our claim.

Proof of Proposition 2.6. Let $s>0, t \geq 0$ be fixed. The idea of the proof is to obtain (2.10) as the Euler equation associated to the functional

$$
\Psi(h):=\int_{X}\left(P_{t}|\nabla h|-\left|\nabla P_{t} h\right|\right) \varphi \mathrm{dm} \quad h \in \operatorname{Lip}(X),
$$

where $\varphi \in \operatorname{Lip}_{\mathrm{bs}}$ is a fixed nonnegative cut-off function. Indeed, thanks to Lemma 2.7 and the Bakry-Émery contraction estimate (1.14), we know that $P_{s} f$ is a minimum of $\Psi$. Thus

$$
\left.\frac{\mathrm{d}}{\mathrm{d} \varepsilon}\right|_{\varepsilon=0} \Psi\left(P_{s} f+\varepsilon g\right)=0 \quad \forall g \in \operatorname{Test}(X, \mathrm{~d}, \mathfrak{m}) .
$$

Notice that the differentiability of $\varepsilon \mapsto \Psi\left(P_{s} f+\varepsilon g\right)$ at $\varepsilon=0$ can be easily checked using $\left|\nabla P_{s} f\right|=$ $P_{s}|\nabla f|>0$. Then we compute

$$
\begin{aligned}
0 & =\left.\frac{\mathrm{d}}{\mathrm{d} \varepsilon}\right|_{\varepsilon=0} \Psi\left(P_{s} f+\varepsilon g\right) \\
& =\left.\frac{\mathrm{d}}{\mathrm{d} \varepsilon}\right|_{\varepsilon=0} \int_{X}\left(P_{t}\left|\nabla P_{s} f+\varepsilon \nabla g\right|-\left|\nabla\left(P_{t+s} f+\varepsilon P_{t} g\right)\right|\right) \varphi \mathrm{d} \mathfrak{m} \\
& =\int_{X}\left(P_{t}\left(\frac{\nabla P_{s} f}{\left|\nabla P_{s} f\right|} \cdot \nabla g\right)-\frac{\nabla P_{t+s} f}{\left|\nabla P_{t+s} f\right|} \cdot \nabla P_{t} g\right) \varphi \mathrm{d} \mathfrak{m} \\
& =\int_{X}\left(P_{t}\left(b_{s} \cdot \nabla g\right)-b_{t+s} \cdot \nabla P_{t} g\right) \varphi \mathrm{d} \mathfrak{m} .
\end{aligned}
$$

The conclusion follows from the arbitrariness of $\varphi$.

As a first consequence of Proposition 2.6 we get the following.

Proposition 2.8. For any $s>0$ it holds $\operatorname{div} b_{s}=0$ and $\nabla_{\mathrm{sym}} b_{s}=0$ according to Definition 1.11. In particular, there exists a regular Lagrangian flow $\boldsymbol{X}^{s}: \mathbb{R} \times X \rightarrow X$ of $b_{s}$ with

$$
\left(\boldsymbol{X}_{t}^{s}\right)_{\#} \mathfrak{m}=\mathfrak{m}, \quad \mathrm{d}\left(\boldsymbol{X}_{t}^{s}(x), \boldsymbol{X}_{t}^{s}(y)\right)=\mathrm{d}(x, y) \quad \forall t \in \mathbb{R}, \quad \forall x, y \in X
$$


Proof. Let $g \in \operatorname{Test}_{c}(X, \mathrm{~d}, \mathfrak{m})$ be fixed. Using (2.10) we obtain

$$
\begin{aligned}
\left|\int_{X} b_{s} \cdot \nabla g(x) \mathrm{d} \mathfrak{m}(x)\right| & =\left|\int_{X} P_{t}\left(b_{s} \cdot \nabla g\right)(x) \mathrm{d} \mathfrak{m}(x)\right| \\
& =\left|\int_{X} b_{t+s} \cdot \nabla P_{t} g(x) \mathrm{d} \mathfrak{m}(x)\right| \\
& \leq \int_{X}\left|\nabla P_{t} g\right|(x) \mathrm{d} \mathfrak{m}(x) .
\end{aligned}
$$

To get $\operatorname{div} b_{s}=0$ it suffices to show that

$$
\lim _{t \rightarrow \infty} \int_{X}\left|\nabla P_{t} g\right|(x) \mathrm{d} \mathfrak{m}(x)=0,
$$

for any nonnegative $g \in \operatorname{Test}_{c}(X, \mathrm{~d}, \mathfrak{m})$. To this aim we use the Gaussian estimates for the heat kernel and its gradient (1.22), (1.23) concluding that there exist a constant $C=C(N)>0$ and $\alpha>1$ such that

$$
\left|\nabla P_{t} g\right|(x) \leq \frac{C}{\sqrt{t}} P_{\alpha t} g(x), \quad \text { for } \mathfrak{m} \text {-a.e. } x \in X .
$$

Let us prove that $\nabla_{\mathrm{sym}} b_{s}=0$ for any $s>0$. First observe that, since $b_{s}$ is divergence-free we have

$$
\int_{X} b_{t+s} \cdot \nabla P_{t} g P_{t} g \mathrm{~d} \mathfrak{m}=\frac{1}{2} \int_{X} b_{t+s} \cdot \nabla\left(P_{t} g\right)^{2} \mathrm{~d} \mathfrak{m}=0,
$$

for any $g \in \operatorname{Test}(X, \mathrm{~d}, \mathfrak{m})$, for any $s>0$ and $t \geq 0$. Using again (2.10) and (2.14) we deduce

$$
\begin{aligned}
0 & =\left.\frac{\mathrm{d}}{\mathrm{d} t}\right|_{t=0} \int_{X} b_{t+s} \cdot \nabla P_{t} g P_{t} g \mathrm{~d} \mathfrak{m}=\left.\frac{\mathrm{d}}{\mathrm{d} t}\right|_{t=0} \int_{X} P_{t}\left(b_{s} \cdot \nabla g\right) P_{t} g \mathrm{~d} \mathfrak{m} \\
& =\int_{X} \Delta\left(b_{s} \cdot \nabla g\right) g \mathrm{~d} \mathfrak{m}+\int_{X} b_{s} \cdot \nabla g \Delta g \mathrm{~d} \mathfrak{m} \\
& =2 \int_{X} b_{s} \cdot \nabla g \Delta g \mathrm{~d} \mathfrak{m},
\end{aligned}
$$

that, by polarization, implies our claim.

The second part of the statement follows from (iv) in Theorem 1.12.

We are now in position to show that $b_{s}$ does not depend on $s>0$.

Lemma 2.9 (Variation formula, version 2). The vector field $b:=b_{s}$ does not depend on $s>0$. In particular, it holds

$$
b \cdot \nabla P_{t} g=P_{t}(b \cdot \nabla g) \quad \mathfrak{m}-\text { a.e. },
$$

for every $g \in \operatorname{Test}(X, \mathrm{~d}, \mathfrak{m})$ and every $t \geq 0$.

The most important ingredient in the proof of Lemma 2.9 is the following lemma.

Lemma 2.10. Let $(X, \mathrm{~d}, \mathfrak{m})$ be an $\operatorname{RCD}(K, \infty)$ m.m.s. and let $T: X \rightarrow X$ be a measure preserving isometry. Then, for any $f \in L^{2}(X, \mathfrak{m})$, it holds

$$
P_{t}(f \circ T)(x)=\left(P_{t} f\right) \circ T(x),
$$

for any $t>0$ and for $\mathfrak{m}$-a.e. $x \in X$.

Proof. We just provide a sketch of the proof since the result is quite standard in the field.

First we observe that, since $T$ is a measure preserving isometry, it holds that $f \in H^{1,2}(X, \mathrm{~d}, \mathfrak{m})$ if and only if $f \circ T \in H^{1,2}(X, \mathrm{~d}, \mathfrak{m})$ and in that case $\mathrm{Ch}(f \circ T)=\mathrm{Ch}(f)$. From this observation we deduce (2.16), since the heat flow is the gradient flow of the Cheeger energy in $L^{2}(X, \mathfrak{m})$. 
Proof of Lemma 2.9. Let $s>0$ and let $\boldsymbol{X}^{s}$, the regular Lagrangian flow associated to $b_{s}$, be fixed.

We know from Proposition 2.8 that for any $t \in \mathbb{R}$ the flow map $\boldsymbol{X}_{t}^{s}$ is a measure preserving isometry of $X$. Therefore, for any $r \geq 0$ and any $g \in \operatorname{Test}(X, \mathrm{~d}, \mathfrak{m})$, using (2.16) with $T=\boldsymbol{X}_{t}^{s}$ and (2.10), we get

$$
\begin{aligned}
\left(b_{s} \cdot \nabla P_{r} g\right) \circ \boldsymbol{X}_{t}^{s} & =\frac{\mathrm{d}}{\mathrm{d} t} P_{r}(g) \circ \boldsymbol{X}_{t}^{s}=\frac{\mathrm{d}}{\mathrm{d} t} P_{r}\left(g \circ \boldsymbol{X}_{t}^{s}\right) \\
& =P_{r}\left(\left(b_{s} \cdot \nabla g\right) \circ \boldsymbol{X}_{t}^{s}\right)=P_{r}\left(b_{s} \cdot \nabla g\right) \circ \boldsymbol{X}_{t}^{s} \\
& =\left(b_{r+s} \cdot \nabla P_{r} g\right) \circ \boldsymbol{X}_{t}^{s} .
\end{aligned}
$$

Since $g$ is arbitrary, the first conclusion in the statement follows. The second one is a direct consequence of Proposition 2.6.

Let us denote by $\boldsymbol{X}$ the regular Lagrangian flow of $b$ from now on, choosing in particular the "good representative" of Theorem 1.12 (iv). Our next aim is to prove that for any $x \in X$ the curve $t \mapsto \boldsymbol{X}_{t}(x)$ is a line. This will yield the sought conclusion about the product structure of $(X, \mathrm{~d}, \mathfrak{m})$ by means of the splitting theorem Theorem 1.13.

Proposition 2.11. For all $s>0$ the identity

$$
P_{s} f\left(\boldsymbol{X}_{-t}(x)\right)=\min _{\bar{B}_{t}(x)} P_{s} f
$$

holds true for any $t \geq 0$ and any $x \in X$.

Before then passing to the proof we wish to explain the heuristic standing behind it with a formal computation:

$$
\frac{\mathrm{d}}{\mathrm{d} t} P_{s} f\left(\boldsymbol{X}_{-t}(x)\right)=-\nabla P_{s} f \cdot \frac{\nabla P_{s} f}{\left|\nabla P_{s} f\right|}\left(\boldsymbol{X}_{-t}(x)\right)=-\left|\nabla P_{s} f\right|\left(\boldsymbol{X}_{-t}(x)\right)=-\left|\nabla\left(P_{s} f \circ \boldsymbol{X}_{t}\right)\right|(x) .
$$

Therefore, setting $u(t, x):=P_{s} f\left(\boldsymbol{X}_{-t}(x)\right)$, it holds that

$$
\partial_{t} u(t, x)+\left|\nabla_{x} u(t, x)\right|=0
$$

and it is well known that the Hopf-Lax semigroup

$$
\mathcal{Q}_{t} u_{0}(x):=\min _{\bar{B}_{t}(x)} u_{0}
$$

provides a solution of (2.18), and the unique viscosity solution (see [N14]). Proposition 2.11 is just telling us that $u(t, x)=P_{s} f\left(X_{-t}(x)\right)$ is precisely the Hopf-Lax semigroup solution.

Proof of Proposition 2.11. Let us denote by $u(t, x)$ the left hand side in (2.17). Since $\mathrm{d}\left(\boldsymbol{X}_{-t}(x), x\right) \leq$ $t$, the inequality $\geq$ in (2.17) is obvious.

Now, we claim that for all $\gamma \in \operatorname{Lip}_{1}([0, \infty) ; X)$ the function $t \mapsto u(t, \gamma(t))$ is nonincreasing. In order to prove the claim, first we observe that $t \mapsto u(t, x)=P_{s} f\left(\boldsymbol{X}_{-t}(x)\right)$ is of class $C^{1}$, since its derivative is $-P_{s}|\nabla f|\left(\boldsymbol{X}_{-t}(x)\right)$ that is a continuous function. Indeed, the validity of this condition for $\mathfrak{m}$-a.e. $x \in X$ follows from the defining conditions of RLF and we can extend it to all $x \in X$ by continuity of the maps $(t, x) \mapsto u(t, x)$ and $(t, x) \mapsto-P_{s}|\nabla f|\left(\boldsymbol{X}_{-t}(x)\right)$. Then by the Leibniz rule in [AGS05, Lemma 4.3.4], it suffices to show that

$$
\limsup _{h \rightarrow 0^{+}} \frac{|u(t, \gamma(t+h))-u(t, \gamma(t))|}{h} \leq P_{s}|\nabla f|\left(\boldsymbol{X}_{-t}(\gamma(t))\right) .
$$

This inequality follows easily from Lemma 2.12 and the inequality $\left|\nabla P_{s} f\right| \leq P_{s}|\nabla f|$, since

$$
\frac{|u(t, \gamma(t+h))-u(t, \gamma(t))|}{h} \leq f_{t}^{t+h} P_{s}|\nabla f|\left(\boldsymbol{X}_{-t}(\gamma(r))\right) \mathrm{d} r,
$$

(here we also used that $r \mapsto \boldsymbol{X}_{-t}(\gamma(r))$ is 1-Lipschitz), by taking the limit as $h \downarrow 0$. 
From the claim, the converse inequality in (2.17) follows easily, because for all $x \in X$ and all minimizers $\bar{x}$ of $P_{s} f$ in $\bar{B}_{t}(x)$ the geodesic property of $(X, \mathrm{~d})$ grants the existence of $\gamma \in$ $\operatorname{Lip}_{1}([0, \infty) ; X)$ with $\gamma(t)=x$ and $\gamma(0)=\bar{x}$. It follows that

$$
u(t, x)=u(t, \gamma(t)) \leq u(0, \gamma(0))=u(0, \bar{x})=P_{s} f(\bar{x})=\min _{\bar{B}_{t}(x)} P_{s} f
$$

Lemma 2.12. Let $(X, \mathrm{~d}, \mathfrak{m})$ be an $\operatorname{RCD}(K, \infty)$ m.m.s. and $u \in \operatorname{Lip}(X)$. Assume that $|\nabla u|$ has a continuous representative in $L^{\infty}(X, \mathfrak{m})$. Then

$$
|u(\gamma(t))-u(\gamma(s))| \leq \int_{s}^{t}|\nabla u|(\gamma(r))\left|\gamma^{\prime}\right|(r) \mathrm{d} r
$$

for any $s<t$ and for any Lipschitz curve $\gamma: \mathbb{R} \rightarrow X$ (where we denoted by $|\nabla u|$ the continuous representative of the minimal relaxed slope of $u$ ).

Proof. To get the sought conclusion we argue by regularization via heat flow as in the proof of [AGS14, Theorem 6.2].

Let $\left(\mu_{r}^{\lambda}\right)_{r \in \mathbb{R}}$ be defined by $\mu_{r}^{\lambda}:=\left(P_{\lambda}\right)^{*} \delta_{\gamma(r)}$. Contractivity yields now that

$$
\begin{aligned}
\left|P_{\lambda} u(\gamma(t))-P_{\lambda} u(\gamma(s))\right| & \leq \int_{s}^{t}\left(\int|\nabla u|^{2} \mathrm{~d} \mu_{r}^{\lambda}\right)^{\frac{1}{2}}\left|\dot{\mu}_{r}^{\lambda}\right| \mathrm{d} r \\
& \leq e^{-K \lambda} \int_{s}^{t}\left(\int|\nabla u|^{2} \mathrm{~d} \mu_{r}^{\lambda}\right)^{\frac{1}{2}}\left|\dot{\gamma}_{r}\right| \mathrm{d} r \\
& =e^{-K \lambda} \int_{s}^{t}\left(P_{\lambda}|\nabla u|^{2}(\gamma(r))\right)^{\frac{1}{2}}\left|\dot{\gamma}_{r}\right| \mathrm{d} r
\end{aligned}
$$

for any $\lambda>0$ and for any $s, t \in \mathbb{R}$. Passing to the limit as $\lambda \downarrow 0$ both the first and the last expression in (2.21) and taking into account the continuity of $u$ and $|\nabla u|$, we obtain (2.20).

By means of Proposition 2.11 we can easily prove the following.

Corollary 2.13. For any $x \in X$ the curve $t \mapsto \boldsymbol{X}_{t}(x)$ is a line, that is to say

$$
\mathrm{d}\left(\boldsymbol{X}_{t}(x), \boldsymbol{X}_{s}(x)\right)=|t-s| \quad \forall s, t \in \mathbb{R} .
$$

Proof. Let us start observing that any $x_{t} \in \bar{B}_{t}(x)$ such that

$$
\min _{y \in \bar{B}_{t}(x)} P_{s} f(y)=P_{s} f\left(x_{t}\right)
$$

has to satisfy $\mathrm{d}\left(x, x_{t}\right)=t$. Otherwise we might replace $x_{t}$ with $\boldsymbol{X}_{-\varepsilon}\left(x_{t}\right)$ (that belongs to $B_{t}(x)$ for $\varepsilon$ sufficiently small) and, since $P_{s} f$ is strictly increasing along the flow lines of $\boldsymbol{X}$, we would get a contradiction.

Furthermore $\boldsymbol{X}_{t}(x) \in \bar{B}_{t}(x)$ since $|b|=1$. Thus it follows from (2.17) that $\mathrm{d}\left(\boldsymbol{X}_{-t}(x), x\right)=t$ for any $t \geq 0$. Using the semigroup property and the fact that $\boldsymbol{X}_{t}$ is an isometry for any $t \in \mathbb{R}$ (see Proposition 2.8) we get the sought conclusion.

Proof of Theorem 2.1. As we anticipated the conclusion that $X$ is isomorphic to $X^{\prime} \times \mathbb{R}$ for some $\mathrm{RCD}(0, N-1)$ m.m.s. $\left(X^{\prime}, \mathrm{d}^{\prime}, \mathfrak{m}^{\prime}\right)$ follows from Corollary 2.13 applying Theorem 1.13.

Let us deal with the second part of the statement.

First of all we claim that all the flow lines of $\boldsymbol{X}$ are vertical lines in $X$, that is to say, denoting by $(z, s) \in X^{\prime} \times \mathbb{R}$ the coordinates on $X, \boldsymbol{X}_{t}(z, s)=(z, t+s)$ for any $z \in X^{\prime}$ and for any $s, t \in \mathbb{R}$. Indeed, since we proved that all integral curves of $b$ are lines in $(X, \mathrm{~d})$, the construction provided by the splitting theorem shows that this is certainly true for a fixed $\bar{z} \in X^{\prime}$. Let us consider any other 
$z \in X^{\prime}$ and call $\boldsymbol{X}_{t}((z, 0))=\left(\boldsymbol{X}_{t}^{1}((z, 0)), \boldsymbol{X}_{t}^{2}(z, 0)\right)$. Taking into account the semigroup property (1.19) and the fact that $\boldsymbol{X}_{t}$ is an isometry for any $t \in \mathbb{R}$, for any $\tau \in \mathbb{R}$ we can compute

$$
\begin{aligned}
\tau^{2}+\mathrm{d}_{Z}^{2}(\bar{z}, z) & =\mathrm{d}^{2}\left(\boldsymbol{X}_{\tau}((\bar{z}, 0)),(z, 0)\right)=\mathrm{d}^{2}\left(\boldsymbol{X}_{t+\tau}((\bar{z}, 0)), \boldsymbol{X}_{t}((z, 0))\right) \\
& =\mathrm{d}^{2}\left((\bar{z}, t+\tau),\left(\boldsymbol{X}_{t}^{1}((z, 0)), \boldsymbol{X}_{t}^{2}((z, 0))\right)\right) \\
& =\left|\left(\boldsymbol{X}_{t}^{2}((z, 0))-t\right)-\tau\right|^{2}+\mathrm{d}_{Z}^{2}\left(\bar{z}, \boldsymbol{X}_{t}^{1}((z, 0))\right) .
\end{aligned}
$$

Since $\tau$ is arbitrary, it easily follows that $\boldsymbol{X}_{t}^{2}((z, 0))=t$ for any $t \in \mathbb{R}$ and therefore $\boldsymbol{X}_{t}^{1}((z, 0))=z$ for any $t \in \mathbb{R}$, as we claimed.

From what we just proved it follows that $\nabla P_{s} f$ is trivial in the $z$ variable and we can conclude that $P_{s} f$ depends only on the $t$-variable for any $s>0$ thanks to the tensorization of the Cheeger energy (see [AGS14, Theorem 6.19]). Passing to the limit as $s \downarrow 0$ we obtain that the same holds true also for $f$.

Knowing that $f$ depends only on the $t$-variable, the monotonicity in this variable can be immediately checked.

\section{Convergence and stability results for sets of finite perime- ter}

In this section we establish some useful compactness and stability results for sequences of sets of finite perimeter defined on a pmGH converging sequence of $\operatorname{RCD}(K, N)$ m.m. spaces. Most of the results we present here adapt and extend to the case of our interest those of [AH17].

Until the end of this section we fix a sequence $\left\{\left(X_{i}, \mathrm{~d}_{i}, \mathfrak{m}_{i}, x_{i}\right)\right\}_{i \in \mathbb{N}}$ of pointed $\operatorname{RCD}(K, N)$ m.m. spaces converging in the pmGH topology to $(Y, \varrho, \mu, y)$ and a proper metric space $\left(Z, \mathrm{~d}_{Z}\right)$ that realizes this convergence according to Definition 1.20.

Since in the rest of the note we will be mainly interested on the case of indicator functions, let us observe that, in that case, we can rephrase the notion of $L^{1}$-strong convergence introduced in Definition 1.26 in the following way.

Definition 3.1. We say that a sequence of Borel sets $E_{i} \subset X_{i}$ such that $\mathfrak{m}_{i}\left(E_{i}\right)<\infty$ for any $i \in \mathbb{N}$ converges in $L^{1}$-strong to a Borel set $F \subset Y$ with $\mu(F)<\infty$ if $\chi_{E_{i}} \mathfrak{m}_{i} \rightarrow \chi_{F} \mu$ in duality with $\mathrm{C}_{\mathrm{bs}}(Z)$ and $\mathfrak{m}_{i}\left(E_{i}\right) \rightarrow \mu(F)$.

We also say that a sequence of Borel sets $E_{i} \subset X_{i}$ converges in $L_{\text {loc }}^{1}$ to a Borel set $F \subset Y$ if $E_{i} \cap B_{R}\left(x_{i}\right) \rightarrow F \cap B_{R}(y)$ in $L^{1}$-strong for any $R>0$.

Remark 3.2. Let us remark that $L^{1}$-strong convergence implies $L_{\text {loc }}^{1}$-strong convergence as a consequence of Lemma 3.5 and the following observation:

$$
\chi_{B_{R}\left(x_{i}\right)} \rightarrow \chi_{B_{R}(y)} \text { in } L^{1} \text {-strong, for any } R>0 .
$$

This convergence property follows from the already remarked fact that spheres have vanishing measure on $\operatorname{RCD}(K, N)$ spaces.

Let us begin with a compactness result which adapts [AH17, Proposition 7.5] to the case of our interest (basically, we add the uniform $L^{\infty}$ bound and this allows to remove the assumption on the existence of a common isoperimetric profile).

Proposition 3.3. Let $\left(X_{i}, \mathrm{~d}_{i}, \mathfrak{m}_{i}, x_{i}\right),(Y, \varrho, \mu, y)$, and $\left(Z, \mathrm{~d}_{Z}\right)$ be as above and fix $r>0$. For any sequence of functions $f_{i} \in \operatorname{BV}\left(X_{i}, \mathfrak{m}_{i}\right)$ such that $\operatorname{supp} f_{i} \subset \bar{B}_{r}\left(x_{i}\right)$ for any $i \in \mathbb{N}$ and

$$
\sup _{i \in \mathbb{N}}\left\{\left|D f_{i}\right|\left(X_{i}\right)+\left\|f_{i}\right\|_{L^{\infty}\left(X_{i}, \mathfrak{m}_{i}\right)}\right\}<\infty,
$$

there exist a subsequence $i(k)$ and $f \in L^{\infty}(Y, \mu) \cap \operatorname{BV}(Y, \varrho, \mu)$ with $\operatorname{supp} f \subset \bar{B}_{r}(y)$ such that $f_{i(k)} \rightarrow f$ in $L^{1}$-strong.

As a corollary, a truncation and a diagonal argument provide a compactness result for sequences of sets with locally uniformly bounded perimeters. 
Corollary 3.4. For any sequence of Borel sets $E_{i} \subset X_{i}$ such that

$$
\sup _{i \in \mathbb{N}} \operatorname{Per}\left(E_{i}, B_{R}\left(x_{i}\right)\right)<\infty \quad \forall R>0
$$

there exist a subsequence $i(k)$ and a Borel set $F \subset Y$ such that $E_{i(k)} \rightarrow F$ in $L_{\text {loc }}^{1}$.

We postpone the proof of Proposition 3.3 and Corollary 3.4 after a technical lemma that will play a role also in the sequel.

Lemma 3.5. Let $\left(X_{i}, \mathrm{~d}_{i}, \mathfrak{m}_{i}, x_{i}\right),(Y, \varrho, \mu, y)$, and $\left(Z, \mathrm{~d}_{Z}\right)$ be as above and $E_{i}, \tilde{E}_{i} \subset X_{i}$ satisfy $\mathfrak{m}_{i}\left(E_{i}\right)+\mathfrak{m}_{i}\left(\tilde{E}_{i}\right)<\infty$. If $E_{i} \rightarrow F$ and $\tilde{E}_{i} \rightarrow \tilde{F}$ in $L^{1}$-strong, for some Borel sets $F, \tilde{F} \subset Y$, then $E_{i} \cap \tilde{E}_{i} \rightarrow F \cap \tilde{F}$ in $L^{1}$-strong.

Proof. Observing that

$$
\chi_{E_{i} \cap \tilde{E}_{i}}=\chi_{E_{i}} \cdot \chi_{\tilde{E}_{i}}=\frac{1}{4}\left[\left(\chi_{E_{i}}+\chi_{\tilde{E}_{i}}\right)^{2}-\left(\chi_{E_{i}}-\chi_{\tilde{E}_{i}}\right)^{2}\right],
$$

the conclusion follows from Proposition 1.28.

Proof of Corollary 3.4. We claim that, possibly extracting a subsequence that we do not relabel, there exist radii $R_{\ell} \uparrow \infty$ as $\ell \rightarrow \infty$ with the following property

$$
\sup _{i \in \mathbb{N}} \operatorname{Per}\left(B_{R_{\ell}}\left(x_{i}\right), X_{i}\right)<\infty \quad \forall l \in \mathbb{N} .
$$

Indeed, applying the coarea formula in the localized version of Corollary 1.9 to the functions $\mathrm{d}\left(x_{i}, \cdot\right)$ and recalling that $\left|\nabla \mathrm{d}\left(x_{i}, \cdot\right)\right|_{i}=1 \mathfrak{m}_{i}$-a.e. for any $i$, we obtain

$$
\int_{0}^{R} \operatorname{Per}\left(B_{r}\left(x_{i}\right), X_{i}\right) \mathrm{d} r=\mathfrak{m}_{i}\left(B_{R}\left(x_{i}\right)\right) \quad \text { for any } R>0 \text { and } i \in \mathbb{N} .
$$

Observing that for any $R>0$ it holds $\mathfrak{m}_{i}\left(B_{R}\left(x_{i}\right)\right) \rightarrow \mu\left(B_{R}(y)\right)$, an application of Fatou's lemma yields now

$$
\int_{0}^{R} \liminf _{i \rightarrow \infty} \operatorname{Per}\left(B_{r}\left(x_{i}\right), X_{i}\right) \mathrm{d} r \leq \liminf _{i \rightarrow \infty} \mathfrak{m}_{i}\left(B_{R}\left(x_{i}\right)\right)=\mu\left(B_{R}(y)\right) \quad \text { for any } R>0 .
$$

The claimed conclusion (3.3) can be obtained from (3.4) via a diagonal argument.

For any $\ell \in \mathbb{N}$ we can now estimate

$$
\sup _{i \in \mathbb{N}} \operatorname{Per}\left(E_{i} \cap B_{R_{\ell}}\left(x_{i}\right), X\right) \leq \sup _{i \in \mathbb{N}} \operatorname{Per}\left(E_{i}, B_{R_{\ell}+1}\left(x_{i}\right)\right)+\sup _{i \in \mathbb{N}} \operatorname{Per}\left(B_{R_{\ell}}\left(x_{i}\right), X\right)<\infty,
$$

thanks to the locality and subadditivity of perimeters (see [A02, pg. 8]) for the first inequality and to (3.2), (3.3) for the second one. Thus for any $\ell \in \mathbb{N}$ we can apply Proposition 3.3 to the functions $f_{i}:=\chi_{E_{i} \cap B_{R_{\ell}}\left(x_{i}\right)}$. Observing that $L^{1}$-strong limits of characteristic functions are characteristic functions (as a consequence of Proposition 1.28), we can use a diagonal argument together with Lemma 3.5 to recover the global limit set.

Proof of Proposition 3.3. Let us fix $t>0$. For any $i \in \mathbb{N}$ we write $f_{i}=P_{t}^{i} f_{i}+\left(f_{i}-P_{t}^{i} f_{i}\right)$ where, for any $i \in \mathbb{N}, P_{t}^{i}$ denotes the heat semigroup on $\left(X_{i}, \mathrm{~d}_{i}, \mathfrak{m}_{i}\right)$. Observe that, as a consequence of the regularizing estimates (1.6), it holds that

$$
\sup _{i \in \mathbb{N}}\left\{\int_{Z}\left|P_{t}^{i} f_{i}\right|^{2} \mathrm{dm}_{i}+\operatorname{Ch}^{i}\left(P_{t}^{i} f_{i}\right)\right\}<+\infty
$$

where $\mathrm{Ch}^{i}$ is the Cheeger energy on $\left(X_{i}, \mathrm{~d}_{i}, \mathfrak{m}_{i}\right)$. Moreover, we claim that

$$
\limsup _{R \rightarrow \infty} \sup _{i \in \mathbb{N}} \int_{Z \backslash B_{R}\left(x_{i}\right)}\left|P_{t}^{i} f_{i}\right|^{2} \mathrm{~d} \mathfrak{m}_{i}=0 \quad \forall t>0 .
$$


Indeed, using both the Gaussian estimates for the heat kernel in (1.22), we get

$$
\begin{aligned}
\int_{Z \backslash B_{R}\left(x_{i}\right)}\left|P_{t}^{i} f_{i}\right|^{2} \mathrm{dm}_{i} \\
\leq\left\|f_{i}\right\|_{L^{\infty}\left(X_{i}, \mathfrak{m}_{i}\right)} \int_{Z \backslash B_{R}\left(x_{i}\right)} P_{t}^{i}\left|f_{i}\right| \mathrm{d} \mathfrak{m}_{i} \\
\leq C\left\|f_{i}\right\|_{L^{\infty}\left(X_{i}, \mathfrak{m}_{i}\right)} \int_{Z \backslash B_{R}\left(x_{i}\right)} \int_{B_{r}\left(x_{i}\right)} \frac{e^{-\frac{\mathrm{d}^{2}(x, y)}{5 t}+c t}}{\mathfrak{m}_{i}\left(B_{\sqrt{t}}(x)\right)}\left|f_{i}(y)\right| \mathrm{d} \mathfrak{m}_{i}(y) \mathrm{d} \mathfrak{m}_{i}(x) \\
\leq C e^{-\frac{(R-r)^{2}}{10 t}}\left\|f_{i}\right\|_{L^{\infty}\left(X_{i}, \mathfrak{m}_{i}\right)} \int_{Z \backslash B_{R}\left(x_{i}\right)} \int_{B_{r}\left(x_{i}\right)} \frac{e^{-\frac{\mathrm{d}^{2}(x, y)}{10 t}+c t}}{\mathfrak{m}_{i}\left(B_{\sqrt{t}}(x)\right)}\left|f_{i}(y)\right| \mathrm{d} \mathfrak{m}_{i}(y) \mathrm{d} \mathfrak{m}_{i}(x) \\
\leq C_{t} e^{-\frac{(R-r)^{2}}{10 t}}\left\|f_{i}\right\|_{L^{\infty}\left(X_{i}, \mathfrak{m}_{i}\right)} \int_{Z} P_{\alpha t}^{i}\left|f_{i}\right| \mathrm{d}_{\mathfrak{m}_{i}} \\
\leq C_{t} e^{-\frac{(R-r)^{2}}{10 t}}\left\|f_{i}\right\|_{L^{\infty}\left(X_{i}, \mathfrak{m}_{i}\right)}\left\|f_{i}\right\|_{L^{1}\left(X_{i}, \mathfrak{m}_{i}\right)},
\end{aligned}
$$

where $\alpha>0$ is a constant depending only on $K$ and $N$.

Taking into account (3.5) and (3.6), we can apply Theorem 1.29 to get that $P_{t}^{i} f_{i}$ admits a subsequence converging in $L^{1}$-strong. In order to conclude the proof it suffices to observe that

$$
\lim _{t \rightarrow 0^{+}} \sup _{i \in \mathbb{N}} \int_{X_{i}}\left|P_{t}^{i} f_{i}-f_{i}\right| \mathrm{d} \mathfrak{m}_{i}=0
$$

as it follows from the inequality

$$
\int_{X_{i}}\left|P_{t}^{i} f_{i}-f_{i}\right| \mathrm{dm}_{i} \leq C(K, t)\left|D f_{i}\right|\left(X_{i}\right)
$$

with $C(K, t) \sim \sqrt{t}$ as $t \rightarrow 0$ (see for instance [AH17, Proposition 6.3]).

Let us pass to a lower semicontinuity result for the total variations.

Proposition 3.6. Let $\left(X_{i}, \mathrm{~d}_{i}, \mathfrak{m}_{i}, x_{i}\right)$ be $\operatorname{RCD}(K, N)$ m.m. spaces converging in the pmGH topology to $(Y, \varrho, \mu, y)$ and $\left(Z, \mathrm{~d}_{Z}\right)$ realizing the convergence as above. Let $f_{i} \in \mathrm{BV}\left(X_{i}, \mathfrak{m}_{i}\right)$ converge in $L^{1}$ strong to $f \in L^{1}(Y, \mu)$. If $\sup _{i}\left|D f_{i}\right|\left(X_{i}\right)<\infty$ then $f \in \mathrm{BV}(Y, \varrho, \mu)$ and

$$
\liminf _{i \rightarrow \infty}\left|D f_{i}\right|\left(X_{i}\right) \geq|D f|(Y)
$$

Furthermore, if

$$
\sup _{i \in \mathbb{N}}\left\|f_{i}\right\|_{L^{\infty}\left(X_{i}, \mathfrak{m}_{i}\right)}<\infty
$$

then

$$
\liminf _{i \rightarrow \infty} \int_{X_{i}} g \mathrm{~d}\left|D f_{i}\right| \geq \int_{Y} g \mathrm{~d}|D f|, \quad \text { for all } g \in \operatorname{Lip}_{\mathrm{bs}}(Z) \text { nonnegative. }
$$

Before than proving Proposition 3.6 we state and prove a simple corollary of it.

Corollary 3.7. Let $\left(X_{i}, \mathrm{~d}_{i}, \mathfrak{m}_{i}, x_{i}\right)$ be $\operatorname{RCD}(K, N)$ m.m. spaces converging in the pmGH topology to $(Y, \varrho, \mu, y)$ and $\left(Z, \mathrm{~d}_{Z}\right)$ realizing the convergence as above. For any $f_{i} \in \operatorname{BV}\left(X_{i}, \mathrm{~d}_{i}, \mathfrak{m}_{i}\right)$ convergent in energy in $\mathrm{BV}$ to $f \in \mathrm{BV}(Y, \varrho, \mu)$ such that $\sup _{i}\left\|f_{i}\right\|_{L^{\infty}\left(X_{i}, \mathfrak{m}_{i}\right)}<+\infty$, it holds that $\left|D f_{i}\right| \rightarrow|D f|$ in duality with $\mathrm{C}_{\mathrm{bs}}(Z)$.

Proof of Corollary 3.7. From (3.9) we can deduce with a standard measure theoretic argument that

$$
\liminf _{i \rightarrow \infty}\left|D f_{i}\right|(A) \geq|D f|(A) \quad \forall A \subset Z \text { open and bounded. }
$$

Let $\nu$ be any weak limit point of $\left|D f_{i}\right|$, in the weak topology induced by $\mathrm{C}_{\mathrm{bs}}(Z)$, along some subsequence $i(k)$ (the sequence $\left|D f_{i}\right|\left(X_{i}\right)$ is bounded and therefore the family $\left\{\left|D f_{i}\right|\right\}_{i}$ is weakly compact). For any open and bounded set $A \subset Z$ such that $\nu(\partial A)=0$, it holds $\lim _{k}\left|D f_{i(k)}\right|(A)=$ 
$\nu(A)$. Hence, taking into account also (3.10), we get $|D f|(A) \leq \nu(A)$. Thus $|D f| \leq \nu$, as measures in $Z$. On the other hand, since the evaluation on open sets is lower semicontinuous w.r.t. the weak convergence induced by $\mathrm{C}_{\mathrm{bs}}(Z)$, by definition of convergence in energy in BV, we have $\nu(Z) \leq \liminf _{k}\left|D f_{i(k)}\right|(Z)=|D f|(Z)$ and therefore $\nu=|D f|$.

Proof of Proposition 3.6. The first part of the statement has been proved in [AH17, Theorem 6.4].

Let us deal with the second one. Fix any $t>0$ and observe that $P_{t}^{i} f_{i} \rightarrow P_{t} f$ in $H^{1,2}$ according to Definition 1.24. Indeed, the $L^{1}$-strong convergence of $f_{i}$ to $f$, combined with (3.8), yields that $f_{i}$ converge in $L^{2}$-strong to $f$ by Proposition 1.28. Therefore we can apply Proposition 1.30 to obtain the claimed conclusion. Hence Proposition 1.25 applies, yielding that

$$
\liminf _{i \rightarrow \infty} \int_{Z} g\left|\nabla P_{t}^{i} f_{i}\right| \mathrm{d} \mathfrak{m}_{i} \geq \int_{Z} g\left|\nabla P_{t} f\right| \mathrm{d} \mu, \quad \text { for all } g \in \operatorname{Lip}_{\mathrm{bs}}(Z) \text { nonnegative. }
$$

In order to prove (3.9) starting from its regularized version (3.11), we argue as in the proof of [AH17, Lemma 5.8]. Taking into account the Bakry-Émery contraction estimate $\left|\nabla P_{t} h\right| \leq e^{-K t} P_{t}^{*}|D h|$ (see (1.14)) and the estimate

$$
\left\|P_{t} g-g\right\|_{L^{\infty}} \leq C(K, N, t) \operatorname{Lip}(g), \quad \text { with } C(K, N, t) \sim \sqrt{t} \text { as } t \rightarrow 0
$$

which is available over any $\operatorname{RCD}(K, N)$ m.m.s. (and can be proved using the Gaussian estimates for the heat kernel (1.22)), we obtain

$$
\begin{aligned}
\liminf _{i \rightarrow \infty} \int_{Z} g \mathrm{~d}\left|D f_{i}\right| & \geq \liminf _{i \rightarrow \infty} \int_{Z} P_{t}^{i} g \mathrm{~d}\left|D f_{i}\right|-\limsup _{i \rightarrow \infty} \int_{Z}\left|P_{t}^{i} g-g\right| \mathrm{d}\left|D f_{i}\right| \\
& \geq e^{K t} \liminf _{i \rightarrow \infty} \int_{Z} g\left|\nabla P_{t}^{i} f_{i}\right| \mathrm{dm}_{i}-C(K, N, t) \operatorname{Lip}(g) \limsup _{i \rightarrow \infty}\left|D f_{i}\right|\left(X_{i}\right) \\
& \geq e^{K t} \int_{Z} g\left|\nabla P_{t} f\right| \mathrm{d} \mu-C(K, N, t) \operatorname{Lip}(g) \limsup _{i \rightarrow \infty}\left|D f_{i}\right|\left(X_{i}\right) .
\end{aligned}
$$

The sought conclusion (3.9) can be obtained passing to the liminf as $t \rightarrow 0$ in (3.12), recalling that $\left|\nabla P_{t} f\right| \mu \rightarrow|D f|$ in duality with $\mathrm{C}_{\mathrm{bs}}(Z)$ as $t \downarrow 0$.

The next result deals with the possibility of approximating in BV energy a set of finite perimeter in the limit space with a sequence of sets of finite perimeter defined on the approximating spaces.

Proposition 3.8. Let $\left(X_{i}, \mathrm{~d}_{i}, \mathfrak{m}_{i}, x_{i}\right)$ be $\operatorname{RCD}(K, N)$ m.m. spaces converging in the pmGH topology to $(Y, \varrho, \mu, y)$ and let $\left(Z, \mathrm{~d}_{Z}\right)$ be realizing the convergence as above. Let $F \subset Y$ be a bounded set of finite perimeter. Then there exists a subsequence $\left(i_{k}\right)$ and (uniformly bounded) sets of finite perimeter $E_{i_{k}} \subset X_{i_{k}}$ such that $\chi_{E_{i_{k}}} \rightarrow \chi_{F}$ in energy in BV as $k \rightarrow \infty$.

Proof. Let us begin observing that the first part of [AH17, Theorem 8.1] provides existence of a sequence $\left(g_{i}\right) \subset \mathrm{BV}\left(X_{i}, \mathfrak{m}_{i}\right)$ strongly converging in BV to $\chi_{F}$. Since by assumption $F \Subset B_{R}(y)$ for some $R>0$, we can find a Lipschitz function $\eta: Z \rightarrow[0,1]$ with support contained in $B_{2 R}(y)$ such that $\left.\eta\right|_{B_{R}(y)} \equiv 1$ and it is easy to check that the sequence $f_{i}:=\eta g_{i}$ still converges in $L^{1}$-weak to $\chi_{F}$ and satisfies $\left|D f_{i}\right| \rightarrow \operatorname{Per}(F)$ as $i \rightarrow \infty$. Furthermore, possibly composing with $\varphi(z):=(z \wedge 1) \vee 0$, using Proposition 1.28 and observing that $\left|D \varphi \circ f_{i}\right|\left(X_{i}\right) \leq\left|D f_{i}\right|\left(X_{i}\right)$ for any $i \in \mathbb{N}$ while $\left|D \varphi \circ \chi_{F}\right|(Y)=\left|D \chi_{F}\right|(Y)$, we can assume that $0 \leq f_{i} \leq 1$ for any $i \in \mathbb{N}$. In particular $\sup _{i \in \mathbb{N}}\left\|f_{i}\right\|_{L^{\infty}\left(X_{i}, \mathfrak{m}_{i}\right)}<+\infty$. Therefore, Proposition 3.3 applies and we obtain that, possibly extracting a subsequence that we do not relabel, $f_{i}$ converge in BV energy to $\chi_{F}$.

Let us now assume, possibly extracting one more subsequence, that the measures $\left(f_{i}\right)_{\#}\left(\chi_{B_{2 R}(y)} \mathfrak{m}_{i}\right)$ weakly converge to some measure $\sigma$ in $[0,1]$. Under this assumption, we claim that $\chi_{\left\{f_{i}>\lambda\right\}}$ still converge to $\chi_{F}$ in $L^{1}$-strong for $\mathscr{L}^{1}$-a.e. $\lambda \in(0,1)$.

In order to prove this claim, we fix $\lambda \in(0,1)$ that is not an atom of $\sigma$, so that

$$
\lim _{\varepsilon \rightarrow 0} \lim _{i \rightarrow \infty} \mathfrak{m}_{i}\left(\left\{\lambda-\varepsilon<f_{i} \leq \lambda\right\}\right)=0 .
$$

From (3.13), using Proposition 1.28, it is immediate to get the $L^{1}$-strong convergence of $\chi_{\left\{f_{i}>\lambda\right\}}$ to $\chi_{F}$ : indeed, it suffices to observe that for all $\varepsilon \in(0, \lambda)$ the functions $\psi_{\varepsilon} \circ f_{i}$ still $L^{1}$-strongly 
converge to $\psi_{\varepsilon} \circ \chi_{F}=\chi_{F}$ for any $\psi$ continuous, identically equal to 0 on $[0, \lambda-\varepsilon]$ and identically equal to 1 on $[\lambda, 1]$. From the $L^{1}$-strong convergence we get, in particular,

$$
\liminf _{i \rightarrow \infty} \operatorname{Per}\left(\left\{f_{i}>\lambda\right\}, X_{i}\right) \geq \operatorname{Per}(F, Y) \quad \text { for } \mathscr{L}^{1} \text {-a.e. } \lambda \in(0,1) .
$$

On the other hand, the coarea formula Theorem 1.8 and the strong convergence of $f_{i}$ yield

$$
\limsup _{i \rightarrow \infty} \int_{0}^{1} \operatorname{Per}\left(\left\{f_{i}>\lambda\right\}, X_{i}\right) \mathrm{d} \lambda=\limsup _{i \rightarrow \infty}\left|D f_{i}\right|\left(X_{i}\right)=\operatorname{Per}(F, Y) .
$$

Thanks to Scheffè's lemma, the combination of (3.14) and (3.15) gives that $\operatorname{Per}\left(\left\{f_{i}>\lambda\right\}, X_{i}\right)$ converge in $L^{1}(0,1)$ to the constant $\operatorname{Per}(F, Y)$. Extracting a subsequence $(i(k))$ pointwise convergent on $(0,1) \backslash I$ with $\mathscr{L}^{1}(I)=0$ and setting $E_{k}=\left\{f_{i(k)}>\lambda\right\} \subset B_{2 R}(y)$ with $\lambda \in(0,1) \backslash I$ and $\sigma(\{\lambda\})=0$, the conclusion is achieved.

Let us conclude this section with a convergence result for quasi-minimal sets of finite perimeter. It will play a key role in the study of blow-ups of sets of finite perimeter we are going to perform in section 4. The strategy of the proof is classical, see for instance [A97, Theorem 4.8].

Proposition 3.9. Let $\left(X_{i}, \mathrm{~d}_{i}, \mathfrak{m}_{i}, x_{i}\right)$ be $\operatorname{RCD}(K, N)$ m.m. spaces converging in the pmGH topology to $(Y, \varrho, \mu, y)$ and let $\left(Z, \mathrm{~d}_{Z}\right)$ be realizing the convergence as above. For any $i \in \mathbb{N}$, let $\lambda_{i} \geq 1$ and let $E_{i} \subset X_{i}$ be a set of finite perimeter satisfying the following $\lambda_{i}$-minimality condition: there exists $R_{i}>0$ such that

$$
\operatorname{Per}\left(E_{i}, X_{i}\right) \leq \lambda_{i} \operatorname{Per}\left(E^{\prime}, X_{i}\right) \quad \forall E^{\prime} \subset X_{i} \text { such that } E_{i} \Delta E^{\prime} \Subset B_{R_{i}}\left(x_{i}\right) .
$$

Assume that, as $i \rightarrow \infty, E_{i} \rightarrow F$ in $L_{\text {loc }}^{1}$ for some set $F \subset Y$ of locally finite perimeter, $\lambda_{i} \rightarrow 1$ and $R_{i} \rightarrow \infty$. Then

(i) $F$ is an entire minimizer of the perimeter (relative to $(Y, \varrho, \mu)$ ), namely

$$
\operatorname{Per}\left(F, B_{r}(y)\right) \leq \operatorname{Per}\left(F^{\prime}, B_{r}(y)\right) \quad \text { whenever } F \Delta F^{\prime} \Subset B_{r}(y) \Subset Y \text { and } r>0 ;
$$

(ii) $\left|D \chi_{E_{i}}\right| \rightarrow\left|D \chi_{F}\right|$ in duality with $\mathrm{C}_{\mathrm{bs}}(Z)$.

Proof. Let us fix $\bar{y} \in Y$ and let $F^{\prime} \subset Y$ be a set of locally finite perimeter satisfying $F \Delta F^{\prime} \Subset B_{r}(\bar{y})$. Let $\bar{x}_{i} \in X_{i}$ converging to $\bar{y}$ in $Z$ and $R>0$ be such that the following properties hold true:

$$
\sup _{i \in \mathbb{N}} \operatorname{Per}\left(B_{R}\left(x_{i}\right), X_{i}\right)<+\infty \quad \text { and } \quad B_{r}\left(\bar{x}_{i}\right) \Subset B_{R}\left(x_{i}\right) \quad \forall i \in \mathbb{N} .
$$

Using Proposition 3.8 we can find a sequence of sets of finite perimeter $E_{i}^{\prime} \subset X_{i}$ converging to $F \cap B_{R}(y)$ in BV energy (note that $F \cap B_{R}(y)$ is a set of finite perimeter thanks to (3.16)).

Let $\nu$ be any weak limit of the sequence of measures with uniformly bounded mass $\left|D \chi_{E_{i}}\right|$. We claim that

$$
\nu\left(B_{s}(\bar{y})\right) \leq \operatorname{Per}\left(F^{\prime}, B_{s}(\bar{y})\right) \text { for } \mathscr{L}^{1} \text {-a.e. } s \in(0, r) .
$$

Before proving (3.17) let us illustrate how to use it to conclude the proof. First of all, notice that (3.10) gives $\nu \geq\left|D \chi_{F}\right|$; if we apply (3.17) with $F^{\prime}=F$ we conclude that $\nu=\left|D \chi_{F}\right|$ locally and then globally, achieving the conclusion (ii) in the statement. The validity of the local minimality condition (i) follows combining the identification $\nu=\left|D \chi_{F}\right|$ with (3.17), letting $s \uparrow r$.

Let us pass to the proof of (3.17). We fix a parameter $s \in(0, r)$ with $\nu\left(\partial B_{s}(\bar{y})\right)=0$, $\operatorname{Per}\left(F^{\prime}, \partial B_{s}(\bar{y})\right)=0$ and set

$$
\tilde{E}_{i}^{s}:=\left(E_{i}^{\prime} \cap B_{s}\left(\bar{x}_{i}\right)\right) \cup\left(E_{i} \backslash B_{s}\left(\bar{x}_{i}\right)\right) .
$$

Using the locality of the perimeter (see [A01, A02]) and the $\lambda_{i}$-minimality of $E_{i}$ (notice that $R_{i} \geq r$ for $i$ big enough), we get

$$
\begin{aligned}
\operatorname{Per}\left(E_{i}, \bar{B}_{s}\left(\bar{x}_{i}\right)\right)= & \operatorname{Per}\left(E_{i}, B_{r}\left(\bar{x}_{i}\right)\right)-\operatorname{Per}\left(E_{i}, B_{r}\left(\bar{x}_{i}\right) \backslash \bar{B}_{s}\left(\bar{x}_{i}\right)\right) \\
\leq & \lambda_{i} \operatorname{Per}\left(\tilde{E}_{i}^{s}, B_{r}\left(\bar{x}_{i}\right)\right)-\operatorname{Per}\left(E_{i}, B_{r}\left(\bar{x}_{i}\right) \backslash \bar{B}_{s}\left(\bar{x}_{i}\right)\right) \\
= & \lambda_{i} \operatorname{Per}\left(\tilde{E}_{i}^{s}, B_{s}\left(\bar{x}_{i}\right)\right)+\lambda_{i} \operatorname{Per}\left(\tilde{E}_{i}^{s}, \partial B_{s}\left(\bar{x}_{i}\right)\right) \\
& +\lambda_{i} \operatorname{Per}\left(\tilde{E}_{i}^{s}, B_{r}\left(\bar{x}_{i}\right) \backslash \bar{B}_{s}\left(\bar{x}_{i}\right)\right)-\operatorname{Per}\left(E_{i}, B_{r}\left(\bar{x}_{i}\right) \backslash \bar{B}_{s}\left(\bar{x}_{i}\right)\right) \\
= & \lambda_{i} \operatorname{Per}\left(E_{i}^{\prime}, B_{s}\left(\bar{x}_{i}\right)\right)+\lambda_{i} \operatorname{Per}\left(\tilde{E}_{i}^{s}, \partial B_{s}\left(\bar{x}_{i}\right)\right)+\left(\lambda_{i}-1\right) \operatorname{Per}\left(E_{i}, B_{r}\left(\bar{x}_{i}\right) \backslash \bar{B}_{s}\left(\bar{x}_{i}\right)\right) .
\end{aligned}
$$


Observe that, taking the limit as $i \rightarrow \infty$, thanks to our choice of $s$, it holds that: $\left(\lambda_{i}-\right.$ 1) $\operatorname{Per}\left(E_{i}, B_{r}\left(\bar{x}_{i}\right) \backslash \bar{B}_{s}\left(\bar{x}_{i}\right)\right) \rightarrow 0, \operatorname{Per}\left(E_{i}, \bar{B}_{s}\left(\bar{x}_{i}\right)\right) \rightarrow \nu\left(B_{s}(\bar{y})\right)$ and eventually $\lambda_{i} \operatorname{Per}\left(E_{i}^{\prime}, B_{s}\left(\bar{x}_{i}\right)\right) \rightarrow$ $\operatorname{Per}\left(F^{\prime}, B_{s}(\bar{y})\right)$, since $\chi_{E_{i}^{\prime}} \rightarrow \chi_{F^{\prime} \cap B_{R}(y)}$ in BV energy and therefore Corollary 3.7 applies. It remains only to prove that

$$
\liminf _{i \rightarrow \infty} \operatorname{Per}\left(\tilde{E}_{i}^{s}, \partial B_{s}\left(\bar{x}_{i}\right)\right)=0, \quad \text { for } \mathscr{L}^{1} \text {-a.e. } s \in(0, r)
$$

Applying (3.22) of Lemma 3.10 below with $f=\chi_{E_{i}^{\prime}}-\chi_{E_{i}}$ we get

$$
\operatorname{Per}\left(\tilde{E}_{i}^{s}, X \backslash \bar{B}_{s}\left(\bar{x}_{i}\right)\right) \leq \int_{X_{i}}\left|\chi_{E_{i}^{\prime}}-\chi_{E_{i}}\right| \mathrm{d}\left|D \chi_{B_{s}\left(\bar{x}_{i}\right)}\right|+\operatorname{Per}\left(E_{i}, X \backslash \bar{B}_{s}\left(\bar{x}_{i}\right)\right) \quad \text { for } \mathscr{L}^{1} \text {-a.e. } s \in(0, r),
$$

that, together with the strong locality the perimeter, yields

$$
P\left(\tilde{E}_{i}^{s}, \partial B_{s}\left(\bar{x}_{i}\right)\right) \leq \int_{X_{i}}\left|\chi_{E_{i}^{\prime}}-\chi_{E_{i}}\right| \mathrm{d}\left|D \chi_{B_{s}\left(\bar{x}_{i}\right)}\right|, \quad \text { for } \mathscr{L}^{1} \text {-a.e. } s \in(0, r) .
$$

Using Fatou's lemma, (3.20), the local version of the coarea formula of Corollary 1.9 and eventually Lemma 3.5 to prove that $\chi_{E_{i}^{\prime}}-\chi_{E_{i}} \rightarrow \chi_{F}-\chi_{F^{\prime}}$ in $L^{1}$-strong, we conclude that

$$
\begin{aligned}
\int_{0}^{r} \liminf _{i \rightarrow \infty} \operatorname{Per}\left(\tilde{E}_{i}^{s}, \partial B_{s}\left(\bar{x}_{i}\right)\right) \mathrm{d} s & \leq \liminf _{i \rightarrow \infty} \int_{0}^{r} \operatorname{Per}\left(\tilde{E}_{i}^{s}, \partial B_{s}\left(\bar{x}_{i}\right)\right) \mathrm{d} s \\
& \leq \liminf _{i \rightarrow \infty} \int_{0}^{r} \int_{X_{i}}\left|\chi_{E_{i}^{\prime}}-\chi_{E_{i}}\right| \mathrm{d}\left|D \chi_{B_{s}\left(\bar{x}_{i}\right)}\right| \\
& =\liminf _{i \rightarrow \infty} \int_{B_{r}\left(\bar{x}_{i}\right)}\left|\chi_{E_{i}^{\prime}}-\chi_{E_{i}}\right| \mathrm{d} \mathfrak{m}_{i}=0
\end{aligned}
$$

therefore yielding (3.19).

Lemma 3.10 (Leibniz rule in $\mathrm{BV})$. Let $(X, \mathrm{~d}, \mathfrak{m})$ be an $\operatorname{RCD}(K, \infty)$ m.m.s. and let $x \in X$. For any $f \in \mathrm{BV}(X, \mathrm{~d}, \mathfrak{m}) \cap L^{\infty}(X, \mathfrak{m})$ and $\mathscr{L}^{1}$-a.e. $r \in(0,+\infty)$ it holds

$$
\left|D\left(f \chi_{B_{r}(x)}\right)\right|(X) \leq \int_{X}|f| \mathrm{d}\left|D \chi_{B_{r}(x)}\right|+|D f|\left(B_{r}(x)\right)
$$

and therefore locality gives

$$
\left|D\left(f \chi_{B_{r}(x)}\right)\right|\left(X \backslash B_{r}(x)\right) \leq \int_{X}|f| \mathrm{d}\left|D \chi_{B_{r}(x)}\right|, \quad \text { for } \mathscr{L}^{1} \text {-a.e. } r \in(0,+\infty) .
$$

Proof. Let us begin observing that the stated conclusion makes sense since, in view of the coarea formula Theorem 1.8, $\int|f| \mathrm{d}\left|D \chi_{B_{r}(x)}\right|$ is well defined for $\mathscr{L}^{1}$-a.e. $r \in(0,+\infty)$.

We divide the proof into two intermediate steps. In the first one we are going to prove that (3.21) holds true under the assumption $f \in \operatorname{Lip}_{\mathrm{b}}(X, \mathrm{~d})$. In the second one we prove the sought inequality passing to the limit the inequalities for regularized functions that we obtained previously.

Step 1. More generally in this step we are going to prove, arguing by regularization on $g$, that, for any $f \in \operatorname{Lip}_{\mathrm{b}}(X, \mathrm{~d})$ and for any nonnegative function $g \in \operatorname{BV}(X, \mathrm{~d}, \mathfrak{m}) \cap L^{\infty}(X, \mathfrak{m})$, it holds

$$
|D(f g)|(X) \leq \int_{X}|f| \mathrm{d}|D g|+\int_{X}|g||\nabla f| \mathrm{d} \mathfrak{m} .
$$

Observe that, if $g \in \operatorname{Lip}_{\mathrm{b}}(X, \mathrm{~d})$ then (3.23) follows from the Leibniz rule. Hence, by the $L^{\infty}-$ Lip regularization of the heat semigroup it follows that, for any $t>0$,

$$
\left|D\left(f P_{t} g\right)\right|(X) \leq \int_{X}|f|\left|\nabla P_{t} g\right| \mathrm{d} \mathfrak{m}+\int_{X} P_{t} g|\nabla f| \mathrm{d} \mathfrak{m} .
$$

The convergence of $P_{t} g$ to $g$ in $L^{1}(X, \mathfrak{m})$ as $t \rightarrow 0$, the lower semicontinuity of the total variation and the Bakry-Émery contraction estimate allow us to pass to the lim inf at the left hand-side and 
to the limit at the right hand-side in (3.24) to get (3.23) (see also the proof of the second step for further details on the limiting procedure).

Step 2. It follows from what we just proved and from the $L^{\infty}-$ Lip regularization property of the heat flow on $\operatorname{RCD}(K, \infty)$ m.m. spaces that, for any $t>0$,

$$
\left|D\left(P_{t} f \chi_{B_{r}(x)}\right)\right|(X) \leq \int_{X}\left|P_{t} f\right| \mathrm{d}\left|D \chi_{B_{r}(x)}\right|+\left|D P_{t} f\right|\left(\bar{B}_{r}(x)\right) \quad \text { for } \mathscr{L}^{1} \text {-a.e. } r \in(0,+\infty) .
$$

Next we observe that $P_{t} f \chi_{B_{r}(x)} \rightarrow f \chi_{B_{r}(x)}$ in $L^{1}(X, \mathfrak{m})$ as $t \rightarrow 0^{+}$and therefore, by the lower semicontinuity of the total variation w.r.t. $L^{1}$ convergence it holds

$$
\left|D\left(f \chi_{B_{r}(x)}\right)\right|(X) \leq \liminf _{t \rightarrow 0^{+}}\left|D\left(P_{t} f \chi_{B_{r}(x)}\right)\right|(X) .
$$

Furthermore, the $L^{1}(X, \mathfrak{m})$ convergence of $P_{t} f$ to $f$ and the coarea formula Theorem 1.8 grant that we can find a sequence $t_{i} \downarrow 0$ in such a way that $P_{t_{i}} f$ converges in $L^{1}\left(X,\left|D \chi_{B_{r}(x)}\right|\right)$ to $f$ for $\mathscr{L}^{1}$-a.e. $r \in(0,+\infty)$. Eventually, let us observe that, due to the Bakry-Émery contraction estimate (1.14),

$$
\limsup _{t \rightarrow 0^{+}}\left|D P_{t} f\right|\left(\bar{B}_{r}(x)\right) \leq \limsup _{t \rightarrow 0^{+}} e^{-K t} P_{t}^{*}|D f|\left(\bar{B}_{r}(x)\right) \leq|D f|\left(\bar{B}_{r}(x)\right), \quad \forall r \in(0,+\infty) .
$$

Passing to the liminf as $t_{i} \downarrow 0$ at the left hand-side of (3.25) taking into account (3.26) and to the limit at the right hand-side taking into account what we observed above, we get the sought estimate (3.21).

\section{Tangents to sets of finite perimeter in $\operatorname{RCD}(K, N)$ spaces}

In this section we study the structure of blow-ups of sets of finite perimeter over $\operatorname{RCD}(K, N)$ metric measure spaces. Inspired by the Euclidean theory developed by De Giorgi in the pioneering papers [DG54, DG55], this can be seen as a first step in a program aimed at understanding the fine structure of sets of finite perimeter.

Before then stating the main results we introduce a definition of tangent for sets of finite perimeter in this abstract setting.

Definition 4.1 (Tangents to a set of finite perimeter). Let $(X, \mathrm{~d}, \mathfrak{m})$ be an $\operatorname{RCD}(K, N)$ m.m.s., $x \in X$ and let $E \subset X$ be a set of locally finite perimeter. We denote by $\operatorname{Tan}_{x}(X, \mathrm{~d}, \mathfrak{m}, E)$ the collection of quintuples $(Y, \varrho, \mu, y, F)$ satisfying the following two properties:

(a) $(Y, \varrho, \mu, y) \in \operatorname{Tan}_{x}(X, \mathrm{~d}, \mathfrak{m})$ and $r_{i} \downarrow 0$ are such that the rescaled spaces $\left(X, r_{i}^{-1} \mathrm{~d}, \mathfrak{m}_{x}^{r_{i}}, x\right)$ converge to $(Y, \varrho, \mu, y)$ in the pointed measured Gromov-Hausdorff topology;

(b) $F$ is a set of locally finite perimeter in $Y$ with $\mu(F)>0$ and, if $r_{i}$ are as in (a), then the sequence $f_{i}=\chi_{E}$ converges in $L_{\text {loc }}^{1}$ to $\chi_{F}$ according to Definition 3.1.

It is clear that the following locality property of tangents holds:

$$
\mathfrak{m}(A \cap(E \Delta F))=0 \quad \Longrightarrow \quad \operatorname{Tan}_{x}(X, \mathrm{~d}, \mathfrak{m}, E)=\operatorname{Tan}_{x}(X, \mathrm{~d}, \mathfrak{m}, F) \quad \forall x \in A .
$$

whenever $E, F$ are sets of locally finite perimeter and $A \subset X$ is open.

We are ready to state the main results of this section.

Theorem 4.2. Let $(X, \mathrm{~d}, \mathfrak{m})$ be an $\operatorname{RCD}(K, N)$ m.m.s. and let $E \subset X$ be a set of locally finite perimeter. For $\left|D \chi_{E}\right|$-a.e. $x \in X$ the set $\operatorname{Tan}_{x}(X, \mathrm{~d}, \mathfrak{m}, E)$ is not empty and for all $(Y, \varrho, \mu, y, F) \in$ $\operatorname{Tan}_{x}(X, \mathrm{~d}, \mathfrak{m}, E)$, one has

$$
\left|\nabla P_{s} \chi_{F}\right| \mu=P_{s}^{*}\left|D \chi_{F}\right| \quad \forall s>0,
$$

where $P_{s}=P_{s}^{Y}$ is the heat semigroup relative to $(Y, \varrho, \mu)$. In particular, for all $t \geq 0$, all functions $f=P_{t} \chi_{F}$ satisfy

$$
\left|\nabla P_{s} f\right|=P_{s}|\nabla f| \quad \mu \text {-a.e. in } Y \text {, for all } s>0 .
$$

Moreover, for each $x \in X$ as above there exists a pointed m.m.s. $\left(Z, \mathrm{~d}_{Z}, \mathfrak{m}_{Z}, \bar{z}\right)$ such that

$$
(Y, \varrho, \mu, y, F)=\left((Z \times \mathbb{R}), \mathrm{d}_{Z} \times \mathrm{d}_{\text {eucl }}, \mathfrak{m}_{Z} \times \mathscr{L}^{1},(\bar{z}, 0),\{t>0\}\right),
$$

where we denoted by $t$ the coordinate of the Euclidean factor in $Z \times \mathbb{R}$. Furthermore: 
(i) if $N \geq 2$ then $Z$ is an $\operatorname{RCD}(0, N-1)$ m.m.s.;

(ii) if $N \in[1,2)$ then $Z$ is a point.

A suitable version of the iterated tangent theorem by Preiss (see Theorem A.1) implies also the following.

Theorem 4.3. Let $(X, \mathrm{~d}, \mathfrak{m})$ be an $\operatorname{RCD}(K, N)$ m.m.s. and let $E \subset X$ be a set of locally finite perimeter. Then $E$ admits a Euclidean half-space as tangent at $x$ for $\left|D \chi_{E}\right|$-a.e. $x \in X$, that is to say

$$
\left(\mathbb{R}^{k}, \mathrm{~d}_{\text {eucl }}, c_{k} \mathscr{L}^{k}, 0^{k},\left\{x_{k}>0\right\}\right) \in \operatorname{Tan}_{x}(X, \mathrm{~d}, \mathfrak{m}, E), \quad \text { for some } k \in[1, N] .
$$

Proof of Theorem 4.3. We claim that the stated conclusion holds true at all points $x \in X$ such that both the iterated tangent property of Theorem A.1 and the rigidity property stated in Theorem 4.2 are satisfied (observe that $\left|D \chi_{E}\right|$-a.e. point satisfies these two properties).

Indeed, if $(Y, \varrho, \mu, y, F) \in \operatorname{Tan}_{x}(X, \mathrm{~d}, \mathfrak{m}, E)$, combining Theorem 4.2 with Theorem 2.1, we can say that $(Y, \varrho, \mu)$ is isomorphic to $Z \times \mathbb{R}$ for some $\operatorname{RCD}(0, N-1)$ m.m.s. $\left(Z, \mathrm{~d}_{Z}, \mathfrak{m}_{Z}\right)$. Furthermore, another consequence of Theorem 2.1 is that $F=\left\{t>t_{0}\right\}$ for some $t_{0} \in \mathbb{R}$, where we denoted by $t$ the coordinate on the Euclidean factor of $Y$. Up to a translation we can also assume that $y=(\bar{z}, 0)$ for some $\bar{z} \in Z$.

We go on observing that, if $i: Z \rightarrow Y$ denotes the canonical inclusion $i(z):=(z, 0)$, it holds $\left|D \chi_{F}\right|=i_{\sharp} \mathfrak{m}_{Z}$ and, for this reason, we shall identify in the sequel $\left|D \chi_{F}\right|$ and $\mathfrak{m}_{Z}$. Moreover, it is easy to check that, if $\left(W, \mathrm{~d}_{W}, \mathfrak{m}_{W}, \bar{w}\right) \in \operatorname{Tan}_{z}\left(Z, \mathrm{~d}_{Z}, \mathfrak{m}_{Z}\right)$, then

$$
\left(W \times \mathbb{R}, \mathrm{d}_{W} \times \mathrm{d}_{\text {eucl }}, \mathfrak{m}_{W} \times \mathscr{L}^{1},(\bar{w}, 0),\{t>0\}\right) \in \operatorname{Tan}_{(z, 0)}(Y, \varrho, \mu, F) .
$$

The sought conclusion can now be obtained choosing $z$ to be a regular point of $\left(Z, \mathrm{~d}_{Z}, \mathfrak{m}_{Z}\right)$ (recall that $\mathfrak{m}_{Z}$-a.e. point of $Z$ is regular), so that $W$ is a Euclidean space of dimension $k \in[0, N-1]$ and applying Theorem A.1 to conclude that

$$
\left(W \times \mathbb{R}, \mathrm{d}_{W} \times \mathrm{d}_{\text {eucl }}, \mathfrak{m}_{W} \times \mathscr{L}^{1},(\bar{w}, 0),\{t>0\}\right) \in \operatorname{Tan}_{x}(X, \mathrm{~d}, \mathfrak{m}, E) .
$$

In the case when the ambient space is non collapsed (see Definition 1.17) one can improve the conclusion of Theorem 4.3 above.

Corollary 4.4. Let $(X, \mathrm{~d}, \mathfrak{m})$ be a $\operatorname{ncRCD}(K, N)$ m.m.s. and let $E \subset X$ a set of locally finite perimeter. Then $\left|D \chi_{E}\right|$ is concentrated on the $N$-dimensional regular set of $X$ and, in addition,

$$
\operatorname{Tan}_{x}(X, \mathrm{~d}, \mathfrak{m}, E)=\left\{\left(\mathbb{R}^{N}, \mathrm{~d}_{\text {eucl }}, c_{N} \mathscr{L}^{N}, 0^{N},\left\{x_{N}>0\right\}\right)\right\} \quad \text { for }\left|D \chi_{E}\right| \text {-a.e. } x \in X .
$$

Proof Corollary 4.4. Observe that Theorem 4.3 gives that $\left|D \chi_{E}\right|$ is concentrated on the set of points where at least one metric measured tangent is Euclidean. In the case when the ambient space is a non collapsed $\operatorname{RCD}(K, N)$ m.m.s. this set coincides with the regular set $\mathcal{R}_{N}$ (see Theorem 1.18 and [DPG18]). To conclude, it remains to invoke Theorem 4.2 and to observe that, in the case of a Euclidean ambient space (4.2) easily yields that $F$ is a half-space (even without appealing to Theorem 2.1).

Remark 4.5. Let us remark that, without the non collapsing assumption, it is not directly possible to improve Theorem 4.3 to Corollary 4.4, as the example mentioned in Remark 1.19 shows. We leave the problem of the concentration of the perimeter measure on the regular set in the general case to a forthcoming work.

Given the statement of Corollary 4.4, it sounds natural to introduce the following.

Definition 4.6. Let $(X, \mathrm{~d}, \mathfrak{m})$ be $\operatorname{ncRCD}(K, N)$ metric measure space and let $E \subset X$ be of locally finite perimeter. Then we define the reduced boundary $\mathcal{F} E$ of $E$ by

$$
\mathcal{F} E:=\left\{x \in \operatorname{supp}\left|D \chi_{E}\right|: \quad \operatorname{Tan}_{x}(X, \mathrm{~d}, \mathfrak{m}, E)=\left\{\left(\mathbb{R}^{N}, \mathrm{~d}_{\text {eucl }}, c_{N} \mathscr{L}^{N}, 0^{N},\left\{x_{N}>0\right\}\right)\right\}\right\} .
$$


It is easily seen that $\mathcal{F} E$ is contained in the essential boundary $\partial^{*} E$, namely the complement of the sets of density and rarefaction. In the more general context of PI spaces it is known after [A02] that $\left|D \chi_{E}\right|$ is representable as $\theta \mathcal{S}\left\llcorner\partial^{*} E\right.$ for some density $\theta$, where $\mathcal{S}$ denotes the measure induced by the gauge function $\zeta\left(B_{r}(x)\right)=\mathfrak{m}\left(B_{r}(x)\right) / r$ with Carathéodory's construction. The following result refines this representation formula in the setting of non collapsed $\operatorname{RCD}(K, N)$ spaces.

Corollary 4.7. Let $(X, \mathrm{~d}, \mathfrak{m})$ be $a \operatorname{ncRCD}(K, N)$ m.m.s. and let $E \subset X$ be a set of locally finite perimeter. Then

$$
\operatorname{Per}(E, \cdot)=\mathcal{S}^{N-1}\llcorner\mathcal{F} E,
$$

where we denote by $\mathcal{S}^{\alpha}$ the $\alpha$-dimensional spherical Hausdorff measure.

Proof. We claim that

$$
\lim _{r \downarrow 0} \sup _{y \in B_{s}(x), s \leq r} \frac{\operatorname{Per}\left(E, B_{s}(y)\right)}{\frac{\omega_{N-1}}{2^{N-1}} \operatorname{diam}{ }^{N-1}\left(B_{s}(y)\right)}=1,
$$

for any $x \in \mathcal{F} E$. Let us write

$$
\begin{aligned}
\frac{\operatorname{Per}\left(E, B_{r}(x)\right)}{\frac{\omega_{N-1}}{2^{N-1}} \operatorname{diam}^{N-1}\left(B_{r}(x)\right)} & =\frac{r \operatorname{Per}\left(E, B_{r}(x)\right)}{\omega_{N-1} C(x, r)} \frac{C(x, r)}{\mathfrak{m}\left(B_{r}(x)\right)} \frac{\mathfrak{m}\left(B_{r}(x)\right)}{r^{N}}\left(\frac{2 r}{\operatorname{diam}\left(B_{r}(x)\right)}\right)^{N-1} \\
& =\frac{\operatorname{Per}^{X_{r}}\left(E, B_{1}(x)\right)}{\omega_{N-1}} \frac{1}{\mathfrak{m}_{x}^{r}\left(B_{1}(x)\right)} \frac{\mathfrak{m}\left(B_{r}(x)\right)}{r^{N}}\left(\frac{2 r}{\operatorname{diam}\left(B_{r}(x)\right)}\right)^{N-1},
\end{aligned}
$$

where $C(x, r)$ is the constant in (1.24). Using the very definition of the reduced boundary $\mathcal{F} E$, the continuity of the diameter w.r.t. GH-convergence, the weak convergence of the perimeters proved in Corollary 4.10 and the fact $\mathfrak{m}\left(B_{r}(x)\right) / r^{N} \rightarrow \omega_{N}$ as $r \rightarrow 0^{+}$when $x \in X$ is regular (see [DPG18, Corollary 1.7]) we get

$$
\liminf _{r \downarrow 0} \sup _{y \in B_{s}(x), s \leq r} \frac{\operatorname{Per}\left(E, B_{s}(y)\right)}{\frac{\omega_{N-1}}{2^{N-1}} \operatorname{diam}^{N-1}\left(B_{s}(x)\right)} \geq \liminf _{r \downarrow 0} \frac{\operatorname{Per}\left(E, B_{r}(x)\right)}{\frac{\omega_{N-1}}{2^{N-1}} \operatorname{diam}^{N-1}\left(B_{r}(x)\right)}=1 .
$$

Let us now prove the converse inequality. In order to do so it suffices to show that, for any sequence of radii $r_{i} \rightarrow 0$ and points $x_{i} \in B_{r_{i}}(x)$, it holds

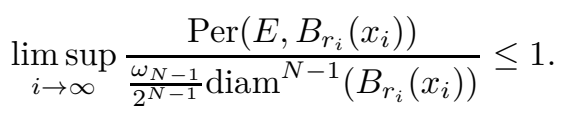

We consider the sequence $\left(X, r_{i}^{-1}, C\left(x, r_{s}\right)^{-1} \mathfrak{m}, x\right)$, that converges to $\left(\mathbb{R}^{N}, \mathrm{~d}_{\text {eucl }}, c_{N} \mathscr{L}^{N}, 0^{N}\right)$, and we assume $x_{i} \rightarrow z \in \bar{B}_{1}\left(0^{N}\right)$. Arguing as in the proof of (4.6), we write

$$
\begin{aligned}
& \frac{\operatorname{Per}\left(E, B_{r_{i}}\left(x_{i}\right)\right)}{\frac{\omega_{N-1}}{2^{N-1}} \operatorname{diam}^{N-1}\left(B_{r_{i}}\left(x_{i}\right)\right)}=\frac{r_{i} \operatorname{Per}\left(E, B_{r_{i}}\left(x_{i}\right)\right)}{\omega_{N-1} C\left(x, r_{i}\right)} \frac{C\left(x, r_{i}\right)}{\mathfrak{m}\left(B_{r_{i}}(x)\right)} \frac{\mathfrak{m}\left(B_{r_{i}}(x)\right)}{r_{i}^{N}}\left(\frac{2 r_{i}}{\operatorname{diam}\left(B_{r_{i}}(x)\right)}\right)^{N-1} \\
& =\frac{\operatorname{Per}^{X_{r_{i}}}\left(E, B_{1}\left(x_{i}\right)\right)}{\omega_{N-1}} \frac{1}{\mathfrak{m}_{x}^{r}\left(B_{1}(x)\right)} \frac{\mathfrak{m}\left(B_{r_{i}}(x)\right)}{r_{i}^{N}}\left(\frac{2 r_{i}}{\operatorname{diam}\left(B_{r_{i}}(x)\right)}\right)^{N-1},
\end{aligned}
$$

and deduce

$$
\lim _{i \rightarrow \infty} \frac{\operatorname{Per}\left(E, B_{r_{i}}\left(x_{i}\right)\right)}{\frac{\omega_{N-1}}{2^{N-1}} \operatorname{diam}^{N-1}\left(B_{r_{i}}\left(x_{i}\right)\right)}=\frac{1}{\omega_{N-1}} \mathscr{H}^{N-1}\left(B_{1}(z) \cap\left\{x_{N}=0\right\}\right) \leq 1 .
$$

The stated conclusion follows now from (4.5) applying [M15, Theorem 5] and checking that $(X, \mathrm{~d})$ is diametrically regular, i.e. for every $x \in X$ and $R>0$ there exists $\delta_{x, R}>0$ such that $\left(0, \delta_{x, R}\right) \ni t \mapsto \operatorname{diam}\left(B_{t}(y)\right)$ is continuous for every $y \in \bar{B}_{R}(x)$ (see the discussion above Theorem 5 in [M15]). To this aim we prove a stronger claim: for any $x \in X$ it holds

$$
\left|\operatorname{diam}\left(B_{t}(x)\right)-\operatorname{diam}\left(B_{s}(x)\right)\right| \leq 2|t-s| \quad \forall t, s \geq 0 .
$$

This property is a consequence of the fact that $X$ is a geodesic metric measure space. Indeed, assuming $t>s$ without loss of generality, for any $y \in B_{t}(x)$ we can find $y^{\prime} \in B_{s}(x)$ realizing 
$\mathrm{d}\left(y, y^{\prime}\right) \leq|t-s|$. In particular, for any $\varepsilon>0$, we can consider $y_{1}, y_{2} \in B_{t}(x)$ such that $\mathrm{d}\left(y_{1}, y_{2}\right) \geq$ $\operatorname{diam}\left(B_{t}(x)\right)-\varepsilon$ and, taking $y_{1}^{\prime}, y_{2}^{\prime} \in B_{s}(x)$ as above, we conclude

$$
\operatorname{diam}\left(B_{t}(x)\right) \leq \mathrm{d}\left(y_{1}, y_{2}\right)+\varepsilon \leq 2|t-s|+\mathrm{d}\left(y_{1}^{\prime}, y_{2}^{\prime}\right)+\varepsilon \leq 2|t-s|+\varepsilon+\operatorname{diam}\left(B_{s}(x)\right),
$$

that implies the sought conclusion letting $\varepsilon \downarrow 0$.

The rest of this section is devoted to the proof Theorem 4.2. First, we are going to prove that tangents are non empty almost everywhere with respect to the perimeter measure, as a consequence of the compactness results developed in section 3 and Proposition 4.9. Then, we will prove that they are rigid, in a suitable sense. This rigidity property will be achieved building mainly on two ingredients: lower semicontinuity and locality of the perimeter and the Bakry-Émery inequality, together with the characterization of its equality cases we obtained in section 2 .

We start stating an asymptotic minimality result that stems from the lower semicontinuity of the perimeter. It has been proved, in a slightly weaker form (namely with a smaller class of competitors $E^{\prime}$ ), first in [A01] under Ahlfors regularity assumption and then, in [A02], for the general case. We refer to the very recent [EGLS18, Theorem 6.1] for the present form. The basic idea originates, to the authors' knowledge, in the work of Fleming [F66] (see also [W89, C99] for variants of this idea in different contexts).

Proposition 4.8 (Asymptotic minimality and doubling). Let (X, d, m) be an $\mathrm{RCD}(K, N)$ m.m.s. and $E \subset X$ be a set of locally finite perimeter. For $\left|D \chi_{E}\right|$-a.e. $x \in X$ there exist $r_{x}>0$ and $\omega_{x}(r):\left(0, r_{x}\right) \rightarrow[0,+\infty)$ such that $\omega_{x}(r) \rightarrow 0$ as $r \rightarrow 0^{+}$and

$$
\operatorname{Per}\left(E, B_{r}(x)\right) \leq\left(1+\omega_{x}(r)\right) \operatorname{Per}\left(E^{\prime}, B_{r}(x)\right)
$$

whenever $E \Delta E^{\prime} \Subset B_{r}(x)$. In addition,

$$
\limsup _{r \rightarrow 0^{+}} \frac{\left|D \chi_{E}\right|\left(B_{2 r}(x)\right)}{\left|D \chi_{E}\right|\left(B_{r}(x)\right)}<+\infty .
$$

Also the following density estimates are important to prove that tangents are almost everywhere non empty. We refer again to [A01, A02] for its proof.

Proposition 4.9. Let $(X, \mathrm{~d}, \mathfrak{m})$ be an $\operatorname{RCD}(K, N)$ m.m.s. and $E \subset X$ be a set of locally finite perimeter. For $\left|D \chi_{E}\right|$-a.e. $x \in X$ it holds

$$
0<\liminf _{r \rightarrow 0^{+}} \frac{r\left|D \chi_{E}\right|\left(B_{r}(x)\right)}{\mathfrak{m}\left(B_{r}(x)\right)} \leq \limsup _{r \rightarrow 0^{+}} \frac{r\left|D \chi_{E}\right|\left(B_{r}(x)\right)}{\mathfrak{m}\left(B_{r}(x)\right)}<+\infty,
$$

and

$$
\liminf _{r \rightarrow 0^{+}} \min \left\{\frac{\mathfrak{m}\left(E \cap B_{r}(x)\right)}{\mathfrak{m}(B(x, r))} ; \frac{\mathfrak{m}\left(E^{c} \cap B_{r}(x)\right)}{\mathfrak{m}(B(x, r))}\right\}>0 .
$$

Corollary 4.10. Let $(X, \mathrm{~d}, \mathfrak{m})$ be an $\operatorname{RCD}(K, N)$ m.m.s. and let $E \subset X$ be a set of locally finite perimeter. Then, for $\left|D \chi_{E}\right|$-a.e. $x \in X$ one has $\operatorname{Tan}_{x}(X, \mathrm{~d}, \mathfrak{m}, E) \neq \emptyset$ and, if $(Y, \varrho, \mu, y, F)$ is as in Definition 4.1, the following properties hold true:

(a) $F$ is an entire minimizer of the perimeter (relative to $(Y, \varrho, \tau)$ ), i.e.

$$
\left|D \chi_{F}\right|\left(B_{r}(y)\right) \leq\left|D \chi_{F^{\prime}}\right|\left(B_{r}(y)\right) \quad \text { whenever } F \Delta F^{\prime} \Subset B_{r}(y) \Subset Y ;
$$

(b) realizing the convergence in a proper metric space $\left(Z, \mathrm{~d}_{Z}\right)$, the perimeters $\left|D^{i} \chi_{E}\right|$ relative to the rescaled spaces in condition (a) of Definition 4.1 weakly converge, in duality with $\mathrm{C}_{\mathrm{bs}}(Z)$, to $\left|D \chi_{F}\right|$.

Proof. Let us consider $x \in X$ such that the statements of Proposition 4.8 and Proposition 4.9 hold true and a sequence of radii $r_{i} \rightarrow 0$ such that $\left(X, r^{-1} \mathrm{~d}, \mu_{x}^{r}, x\right) \rightarrow(Y, \varrho, \mu, y)$ in the pmGH topology. Thanks to (4.9) and Corollary 3.4 with $\chi_{E_{i}}=\chi_{E}$, possibly extracting a subsequence we can assume that there exists a set $F \subset Y$ with locally finite perimeter such that $\chi_{E} \rightarrow \chi_{F}$ in $L_{\text {loc }}^{1}$. Note that $\mu(F)>0$ thanks to (4.10). This implies that $(Y, \varrho, \mu, y, F) \in \operatorname{Tan}(E, x)$. To achieve (a) and (b) it is enough to apply Proposition 3.9, recalling (4.7). 
The next key result to prove Theorem 4.2 is Proposition 4.12. Before stating and proving it we need a technical lemma.

Lemma 4.11. Let $\left(X_{n}, \mathrm{~d}_{n}, \mathfrak{m}_{n}\right)$ be $\operatorname{RCD}(K, N)$ m.m. spaces $m G H$ converging to $(Y, \varrho, \mu)$ and assume that the convergence is realized into a proper metric space $\left(Z, \mathrm{~d}_{Z}\right)$. Let $\eta_{n}, \eta$ be nonnegative Borel measures giving finite mass to bounded sets, such that $\operatorname{supp} \eta_{n} \subset \operatorname{supp} \mathfrak{m}_{n}, \operatorname{supp} \eta \subset \operatorname{supp} \mu$ and $\eta_{n}$ weakly converge to $\eta$ in duality with $\mathrm{C}_{\mathrm{bs}}(Z)$. Then

$$
P_{t}^{Y} \eta(x) \leq \liminf _{n \rightarrow \infty} P_{t}^{n} \eta_{n}\left(x_{n}\right), \quad \text { for any } t>0 \text { and for any } \operatorname{supp} \mathfrak{m}_{n} \ni x_{n} \rightarrow x \in \operatorname{supp} \mu \text {. }
$$

Proof. In [AHT18, Theorem 3.3], building on [GMS15], it is proved that, denoting by $p^{n}$ and $p^{Y}$ the heat kernels of $\left(X_{n}, \mathrm{~d}_{n}, \mathfrak{m}_{n}\right)$ and $(Y, \varrho, \mu)$ respectively, it holds

$$
\lim _{n \rightarrow \infty} p_{t}^{n}\left(x_{n}, y_{n}, t\right)=p_{t}^{Y}(x, y), \quad \text { for any } t>0,
$$

whenever $\operatorname{supp} \mathfrak{m}_{n} \times \operatorname{supp} \mathfrak{m}_{n} \ni\left(x_{n}, y_{n}\right) \rightarrow(x, y) \in \operatorname{supp} \mu \times \mu$. Since

$$
P_{t}^{Y} \eta(x)=\int p_{t}^{Y}(x, y) \mathrm{d} \eta(y) \quad \text { and } \quad P_{t}^{n} \eta_{n}\left(x_{n}\right)=\int p_{t}^{n}\left(x_{n}, y\right) \mathrm{d} \eta(y)
$$

the validity of (4.11) follows from Lemma 1.3 and Fatou's lemma with the obvious choice for the weakly convergent sequence of measures and $f_{n}(\cdot):=p_{t}^{n}\left(x_{n}, \cdot\right), f:=p_{t}(x, \cdot)$, which satisfy the lower semicontinuity condition (1.2) in view of (4.12).

Proposition 4.12. Let $E \subset X$ be a set of finite perimeter and let $(Y, \varrho, \mu, y, F) \in \operatorname{Tan}_{x}(X, \mathrm{~d}, \mathfrak{m}, E)$ for some $x \in X$. Let $r_{i} \downarrow 0$ be a sequence of radii realizing the convergence in Definition 4.1. Then

$$
\left|\nabla^{i} P_{t}^{i} \chi_{E}\right| \mathfrak{m}_{i} \rightarrow\left|\nabla^{Y} P_{t}^{Y} \chi_{F}\right| \mu \quad \text { in duality with } \mathrm{C}_{\mathrm{bs}}(Z) \text {, for any } t>0 \text {. }
$$

Proof. We wish to implement a strategy very similar to the one adopted in the proof of Proposition 1.30 (see [AH17, Theorem 5.4, Corollary 5.5] and [GMS15]).

Let us begin proving that, for any $\operatorname{supp}_{\mathfrak{m}_{i}} \ni x_{i} \rightarrow x \in \operatorname{supp} \mu$ and for any $t>0$, it holds

$$
\lim _{i \rightarrow \infty} P_{t}^{i} \chi_{E}\left(x_{i}\right)=P_{t}^{Y} \chi_{F}(x) .
$$

To this aim we first observe that, by the very definition of tangent, it holds that $\chi_{E} \mathfrak{m}_{n} \rightarrow \chi_{F} \mu$ in duality with $\mathrm{C}_{\mathrm{bs}}(Z)$ and therefore Lemma 4.11 yields

$$
P_{t}^{Y} \chi_{F}(x) \leq \liminf _{i \rightarrow \infty} P_{t}^{i} \chi_{E}\left(x_{i}\right)
$$

Moreover, since $\left(1-\chi_{E}\right) \mathfrak{m}_{n} \rightarrow\left(1-\chi_{F}\right) \mu$ in duality with $\mathrm{C}_{\mathrm{bs}}(Z)$, applying Lemma 4.11 once more and with a simple algebraic manipulation, we obtain

$$
\limsup _{i \rightarrow \infty} P_{t}^{i} \chi_{E}\left(x_{i}\right) \leq P_{t}^{Y} \chi_{F}(x) .
$$

Combining (4.14) with (4.15) we obtain (4.13).

Let us proceed observing that, in view of the quantitative form of the $L^{\infty}$-Lip regularization on $\operatorname{RCD}(K, \infty)$ spaces provided by $(1.15)$, for any $t>0$ the functions $P_{t}^{i} \chi_{E}$ and $P_{t}^{Y} \chi_{F}$ are uniformly Lipschitz.

Fix now reference points $y \in Y$ and $X_{i} \ni x_{i} \rightarrow y$. Building upon [MN14, Lemma 3.1], for any $R>0$ it is possible to find Lipschitz cut-off functions $\eta_{R}: Y \rightarrow[0,1], \eta_{R}^{i}: X_{i} \rightarrow[0,1]$ such that $\operatorname{supp} \eta_{R} \subset B_{2 R}^{Y}(y), \operatorname{supp} \eta_{R}^{i} \subset B_{2 R}^{i}\left(x_{i}\right),\left.\eta_{R}\right|_{B_{R}^{Y}(y)} \equiv 1,\left.\eta_{R}^{i}\right|_{B_{R}^{i}\left(x_{i}\right)} \equiv 1$, uniformly Lipschitz, with uniformly bounded laplacians and such that $\eta_{R}^{i}$ converge to $\eta_{R}$ both pointwise and $L^{2}$-strongly. We remark indeed that, in view of [AHT18, Proposition 3.2], pointwise and $L^{2}$-strong convergence are equivalent for uniformly bounded, uniformly continuous and uniformly boundedly supported functions. Let us observe that, if we are able to prove that

$$
f_{i}:=\eta_{R}^{i} P_{t}^{i} \chi_{E} \rightarrow \eta_{R} P_{t}^{Y} \chi_{F}=: f \quad \text { strongly in } H^{1,2} \text { for all } R>0,
$$


the conclusion will follow from the locality of the minimal weak upper gradient and Theorem 1.31, which grants the $L^{1}$-strong convergence of $\left|\nabla^{i}\left(\eta_{R}^{i} P_{t}^{i} \chi_{E}\right)\right|^{2}$ to $\left|\nabla^{Y} \eta_{R} P_{t}^{Y} \chi_{F}\right|^{2}$ (that we can improve to $L^{1}$-strong convergence of $\left|\nabla^{i}\left(\eta_{R}^{i} P_{t}^{i} \chi_{E}\right)\right|$ to $\left|\nabla^{Y} \eta_{R} P_{t}^{Y} \chi_{F}\right|$ in view of the uniform Lipschitz bounds and of Proposition 1.28).

In order to prove the above claimed convergence, we begin observing that $f_{i}$ converge pointwise to $f$ by (4.13) and the very construction of the family of cut-off functions $\eta_{R}^{i}$. Therefore, taking into account the uniform Lipschitz bounds, the uniform boundedness and the uniform bounds on the supports, $f_{i} \rightarrow f$ strongly in $L^{2}$ by [AHT18, Proposition 3.2]. To improve the convergence from $L^{2}$-strong to $H^{1,2}$-strong we wish to apply Proposition 1.30. In order to do so, it remains to prove that $\Delta f_{i}$ are uniformly bounded in $L^{2}$. To this aim we compute

$$
\Delta f_{i}=\Delta \eta_{R}^{i} P_{t}^{i} \chi_{E}+2 \nabla \eta_{R}^{i} \cdot \nabla P_{t}^{i} \chi_{E}+\eta_{R}^{i} \Delta P_{t}^{i} \chi_{E}
$$

and observe that all the terms at the right hand side in (4.16) are uniformly bounded in $L^{2}$ in view of the uniform $L^{\infty}$ bounds on values, minimal weak upper gradients and laplacians of the cut-off functions, the uniform $L^{\infty}$ and Lipschitz bounds on $P_{t}^{i} \chi_{E}$ and the regularizing estimate for the Laplacian under heat flow in (1.6).

We are now ready to prove Theorem 4.2.

Proof of Theorem 4.2. Let us consider the case when $E$ has finite perimeter. The generalization to sets of locally finite perimeter can be obtained building upon Lemma 3.10 and (4.1), arguing in a standard way.

Recall that the $B V$-version (1.14) of the 1-Bakry-Émery contraction estimate gives

$$
\left|\nabla P_{t} \chi_{E}\right| \mathfrak{m} \leq e^{-K t} P_{t}^{*}\left|D \chi_{E}\right| \quad \forall t>0 .
$$

Let $h_{t}: X \rightarrow[0,1]$ be the density of $e^{K t}\left|\nabla P_{t} \chi_{E}\right| \mathfrak{m}$ with respect to $P_{t}^{*}\left|D \chi_{E}\right|$. Then, one has

$$
\int_{X}\left(1-P_{t} h_{t}\right) \mathrm{d}\left|D \chi_{E}\right|=\left|D \chi_{E}\right|(X)-\int_{X} h_{t} d P_{t}^{*}\left|D \chi_{E}\right|=\left|D \chi_{E}\right|(X)-e^{K t} \int_{X}\left|\nabla P_{t} \chi_{E}\right| \mathrm{dm} .
$$

By lower semicontinuity, this proves that $g_{t}:=1-P_{t} h_{t}$ converges to 0 strongly in $L^{1}\left(X,\left|D \chi_{E}\right|\right)$.

Now, setting for simplicity of notation $\nu=\left|D \chi_{E}\right|$, we claim that

$$
\lim _{t \downarrow 0} \frac{1}{\nu\left(B_{R \sqrt{t}}(x)\right)} \int_{B_{R \sqrt{t}}(x)} g_{t} d \nu=0 \quad \forall R>0, \text { for } \nu \text {-a.e. } x \in X .
$$

Thanks to the asymptotic doubling property (4.8), it is sufficient to prove the result $\nu$-a.e. on a Borel set $F$ with this property: for some $L>0$, for all $x \in F$ and $0<r<1 / L$ one has $\nu\left(B_{5 r}(x)\right) \leq L \nu\left(B_{r}(x)\right)$. By Vitali's theorem, it follows that the localized maximal function

$$
M|g|(x):= \begin{cases}\sup _{r \in(0,1 / L)} \frac{\int_{B_{r}(x)}|g| \mathrm{d} \nu}{\nu\left(B_{r}(x)\right)} & \text { if } x \in F ; \\ 0 & \text { if } x \in X \backslash F ;\end{cases}
$$

satisfies

$$
\nu(\{M|g|>\tau\}) \leq \frac{L}{\tau} \int|g| \mathrm{d} \nu \quad \forall \tau>0 .
$$

Let us apply this estimate to the functions $g_{t}=1-P_{t} h_{t}$ : given $\varepsilon>0$, for $t<t(\varepsilon)$ one has $\int g_{t} \mathrm{~d} \nu<\varepsilon^{2}$, and then $\nu\left(\left\{M g_{t}>\varepsilon\right\}\right) \leq L \varepsilon$. We obtain that

$$
\int_{B_{r}(x)} g_{t} \mathrm{~d} \nu \leq \varepsilon \nu\left(B_{r}(x)\right) \quad \text { for } r<\frac{1}{L}, t<t(\varepsilon)
$$

for all $x \in F_{\varepsilon} \subset F$, with $\mu\left(F \backslash F_{\varepsilon}\right)$ smaller than $L \varepsilon$. In particular, on $F_{\varepsilon}$ one has

$$
\limsup _{t \downarrow 0} \frac{1}{\nu\left(B_{R \sqrt{t}}(x)\right)} \int_{B_{R \sqrt{t}}(x)} g_{t} d \nu \leq \varepsilon \quad \forall R>0 .
$$


Since $\varepsilon$ is arbitrary, we have proved that (4.17) holds $\nu$-a.e. on $F$.

The claimed conclusion (4.2) will be achieved through two intermediate steps starting from (4.17).

First, let us observe that, for any $R, s, t>0$ and for any $x \in X$, it holds

$$
\frac{1}{\nu\left(B_{R \sqrt{t}}(x)\right)} \int_{B_{R \sqrt{t}}(x)} g_{t s} \mathrm{~d} \nu=\frac{1}{\left|D^{t} \chi_{E}\right|\left(B_{R}^{t}(x)\right)} \int_{B_{R}^{t}(x)} P_{s}^{t}\left(1-e^{K t} \frac{\left|\nabla^{t} P_{s}^{t} \chi_{E}\right|}{\left(P_{s}^{t}\right)^{*}\left|D^{t} \chi_{E}\right|}\right) \mathrm{d}\left|D^{t} \chi_{E}\right|,
$$

where we denoted by $P^{t}, \nabla^{t}, D^{t}$ and $B^{t}$ the heat semigroup, the minimal weak upper gradients, the total variation measure and the balls associated to the rescaled metric measure structure $\left(X, \sqrt{t}^{-1} \mathrm{~d}, \mathfrak{m}_{x}^{\sqrt{t}}, x\right)$ and we are identifying measures absolutely continuous w.r.t. the reference one with their densities.

Step 1. We claim that, if $(Y, \varrho, \mu, y, F) \in \operatorname{Tan}_{x}(X, \mathrm{~d}, \mathfrak{m}, E)$ and $t_{i} \downarrow 0$ is a sequence realizing the convergence in Definition 4.1, then

$$
\int P_{s}\left(1-\frac{\left|\nabla P_{s} \chi_{F}\right|}{P_{s}^{*}\left|D \chi_{F}\right|}\right) \mathrm{d} \eta_{R} \leq \liminf _{i \rightarrow \infty} \int P_{s}^{t_{i}}\left(1-e^{K s t_{i}} \frac{\left|\nabla^{t_{i}} P_{s}^{t_{i}} \chi_{E}\right|}{\left(P_{s}^{t_{i}}\right)^{*}\left|D^{t_{i}} \chi_{E}\right|}\right) \mathrm{d} \eta_{R}^{i}
$$

for $\mathscr{L}^{1}$-a.e. $R>0$, where

$$
\left\{\begin{array}{l}
\eta_{R}:=\frac{1}{\left|D \chi_{F}\right|\left(B_{R}(y)\right)}\left|D \chi_{F}\right|\left\llcorner B_{R}(y),\right. \\
\eta_{R}^{i}:=\frac{1}{\left|D^{t_{i}} \chi_{E}\right|\left(B_{R}^{t_{i}}(x)\right)}\left|D^{t_{i}} \chi_{E}\right|\left\llcorner B_{R}^{t_{i}}(x) .\right.
\end{array}\right.
$$

In order to prove (4.19), we begin observing that $\eta_{R}^{i}$ weakly converges to $\eta_{R}$ for $\mathscr{L}^{1}$-a.e. $R>0$. Therefore, the validity of (4.19) will follow from Lemma 1.3 if we prove that

$$
P_{s}\left(1-\frac{\left|\nabla P_{s} \chi_{F}\right|}{\left(P_{s}\right)^{*}\left|D \chi_{F}\right|}\right)(w) \leq \liminf _{i \rightarrow \infty} P_{s}^{t_{i}}\left(1-e^{K s t_{i}} \frac{\left|\nabla^{t_{i}} P_{s}^{t_{i}} \chi_{E}\right|}{\left(P_{s}^{t_{i}}\right)^{*}\left|D^{t_{i}} \chi_{E}\right|}\right)\left(w_{i}\right),
$$

whenever $w_{i} \in X_{i} \rightarrow w \in Y$. Let us observe that, for any $\phi \in \mathrm{C}_{\mathrm{bs}}(Z)$, it holds

$$
\limsup _{i \rightarrow \infty} e^{K s t_{i}} \int \phi \frac{\left|\nabla^{t_{i}} P_{s}^{t_{i}} \chi_{E}\right|}{\left(P_{s}^{t_{i}}\right)^{*}\left|D^{t_{i}} \chi_{E}\right|} \mathrm{dm} \mathfrak{m}_{i} \leq \int \phi \frac{\left|\nabla P_{s} \chi_{F}\right|}{P_{s}^{*}\left|D \chi_{F}\right|} \mathrm{d} \mu \text {. }
$$

Indeed, by Proposition 4.12, $\left|\nabla^{t_{i}} P_{s}^{t_{i}} \chi_{E}\right| \mathfrak{m}_{i}$ weakly converge to $\left|\nabla P_{s} \chi_{F}\right| \mu$ in duality with $\mathrm{C}_{\mathrm{bs}}(Z)$, and the functions

$$
f_{i}:=\frac{\phi}{\left(P_{s}^{t_{i}}\right)^{*}\left|D^{t_{i}} \chi_{E}\right|} \quad \text { and } \quad f:=\frac{\phi}{P_{s}^{*}\left|D \chi_{F}\right|}
$$

are continuous, have uniformly bounded supports and satisfy the upper semicontinuity property (1.1) thanks to Lemma 4.11 (recall that $\left|D^{t_{i}} \chi_{E}\right|$ weakly converge to $\left|D \chi_{F}\right|$ in duality with $\mathrm{C}_{\mathrm{bs}}(Z)$ ). Hence (4.20) and then (4.19) follow from Lemma 1.1, taking into account also Remark 1.2.

Step 2. We can now prove (4.2). If we choose $x \in X$ such that (4.17) holds true (we proved above that $\left|D \chi_{E}\right|$-a.e. $x \in X$ has this property), combining (4.18) with (4.19), we obtain

$$
\int_{B_{R}(y)} P_{s}\left(1-\frac{\left|\nabla P_{s} \chi_{F}\right|}{P_{s}^{*}\left|D \chi_{F}\right|}\right) \mathrm{d}\left|D \chi_{F}\right| \leq 0 .
$$

Observing that, by gradient contractivity on the $\operatorname{RCD}(0, N)$ space $(Y, \varrho, \mu)$, it holds

$$
1-\frac{\left|\nabla P_{s} \chi_{F}\right|}{P_{s}^{*}\left|D \chi_{F}\right|} \geq 0 \quad \mu \text {-a.e. on } Y
$$


we can let $R \rightarrow \infty$ in (4.22) to get

$$
\int P_{s}\left(1-\frac{\left|\nabla P_{s} \chi_{F}\right|}{P_{s}^{*}\left|D \chi_{F}\right|}\right) \mathrm{d}\left|D \chi_{F}\right|=0 .
$$

Then, using once more the sign property (4.23), we obtain (4.2).

Combining the just proved rigidity (4.2) with Theorem 2.1, we can say that $(Y, \varrho, \mu)$ is isomorphic to $Z \times \mathbb{R}$ for some $\operatorname{RCD}(0, N-1)$ m.m.s. $\left(Z, \mathrm{~d}_{Z}, \mathfrak{m}_{Z}\right)$. Furthermore, another consequence of Theorem 2.1 is that $F=\left\{t>t_{0}\right\}$ for some $t_{0} \in \mathbb{R}$, where we denoted by $t$ the coordinate on the Euclidean factor of $Y$. Up to a translation we can also assume that $y=(\bar{z}, 0)$ for some $\bar{z} \in Z$.

\section{A Appendix}

In this appendix we prove a version of the iterated tangent theorem by Preiss (see [P87]). The proof is inspired by those of [GMR15, Theorem 3.2] and [AKL08, Theorem 6.4], dealing with pmGH tangents to $\operatorname{RCD}(K, N)$ spaces and tangents to sets of finite perimeters over Carnot groups, respectively (see also [LD11] for a previous result regarding pGH-tangents of metric spaces equipped with a doubling measure).

Theorem A.1. Let $(X, \mathrm{~d}, \mathfrak{m})$ be an $\operatorname{RCD}(K, N)$ m.m.s. and let $E \subset X$ be a set of finite perimeter. Then for $\left|D \chi_{E}\right|$-a.e. $x \in X$ the following property holds true: for every $(Y, \varrho, \mu, y, F) \in$ $\operatorname{Tan}_{x}(X, \mathrm{~d}, \mathfrak{m}, E)$ one has

$$
\operatorname{Tan}_{y^{\prime}}(Y, \varrho, \mu, F) \subset \operatorname{Tan}_{x}(X, \mathrm{~d}, \mathfrak{m}, E) \quad \text { for every } y^{\prime} \in \operatorname{supp}\left|D \chi_{F}\right| .
$$

Thanks to Corollary 4.10 we need only to prove the result at $\left|D \chi_{E}\right|$-a.e. $x \in X$ for all $(Y, \varrho, \mu, y, F) \in \operatorname{Tan}_{x}^{*}(X, \mathrm{~d}, \mathfrak{m}, E)$, where $\operatorname{Tan}_{x}^{*}(X, \mathrm{~d}, \mathfrak{m}, E)$ is defined adding to the conditions in Definition 4.1 the condition (b) of Corollary 4.10, namely that the perimeter measures of the rescaled spaces weakly converge, in the duality with $\mathrm{C}_{\mathrm{bs}}(X)$, to the perimeter measure of $F$.

Let us briefly recall the notion of outer measure and its main properties. Given a positive measure $\mu$ over a metric space $(X, \mathrm{~d})$ we set

$$
\mu^{*}(A):=\inf \{\mu(B): B \text { Borel, } A \subset B\}, \quad \forall A \subset X .
$$

It is immediate to see that $\mu^{*}$ is countably sub-additive. Let us remark that if $\mu$ is asymptotically doubling then

$$
\lim _{r \downarrow 0} \frac{\mu^{*}\left(A \cap B_{r}(x)\right)}{\mu\left(B_{r}(x)\right)}=1 \quad \text { for } \mu^{*} \text {-a.e. } x \in A .
$$

Indeed, we can find a set $B \in \mathscr{B}(X)$ containing $A$ such that $\mu(B)=\mu^{*}(A)$, so that $\mu^{*}(C \cap A)=$ $\mu(C \cap B)$ for every $C \in \mathscr{B}(X)$. In particular, taking $C=B_{r}(x)$, we have

$$
\lim _{r \downarrow 0} \frac{\mu^{*}\left(A \cap B_{r}(x)\right)}{\mu\left(B_{r}(x)\right)}=\lim _{r \downarrow 0} \frac{\mu\left(B \cap B_{r}(x)\right)}{\mu\left(B_{r}(x)\right)}=1,
$$

for every $x \in B$ of density 1 for the measure $\mu$. Since $\mu$ is asymptotically doubling, $\mu$-a.e $x \in B$ has this property and (A.2) follows.

Let us start with a technical lemma.

Lemma A.2. Let $(X, \mathrm{~d}, \mathfrak{m})$ and let $E \subset X$ be as in the assumptions of Theorem A.1. Let $A \subset X$ and $x \in A$ be such that

$$
\lim _{r \downarrow 0} \frac{\left|D \chi_{E}\right|^{*}\left(A \cap B_{r}(x)\right)}{\left|D \chi_{E}\right|\left(B_{r}(x)\right)}=1,
$$

where $\left|D \chi_{E}\right|^{*}$ is the outer measure associated to $\left|D \chi_{E}\right|$ according to (A.1). Assume that $(Y, \varrho, \mu, F) \in$ $\operatorname{Tan}_{x}^{*}(X, \mathrm{~d}, \mathfrak{m}, E)$ and consider

$$
\begin{aligned}
& \Psi_{i}:\left(X, r_{i}^{-1} \mathrm{~d}\right) \rightarrow\left(Z, \mathrm{~d}_{Z}\right) \quad \forall i \in \mathbb{N}, \\
& \Psi:\left(Y, \mathrm{~d}_{Y}\right) \rightarrow\left(Z, \mathrm{~d}_{Z}\right),
\end{aligned}
$$


a family of isometries realizing the pmGH convergence as in Definition 1.20. Then, for any $y^{\prime} \in$ $\operatorname{supp}\left|D \chi_{F}\right|$, there exists a sequence $\left(x_{i}\right) \subset A$ such that

$$
\lim _{i \rightarrow \infty} \mathrm{d}_{Z}\left(\Psi_{i}\left(x_{i}\right), \Psi\left(y^{\prime}\right)\right)=0 .
$$

Roughly speaking, Lemma A.2 tells us that it is possible to approximate every point in the support of any tangent by means of points in $A$, whenever $A$ is "large" in a measure-theoretic sense.

Proof of Lemma A.2. As a first step we show the existence of an auxiliary sequence $\left(x_{i}\right) \subset X$, satisfying $\lim _{i} \mathrm{~d}_{Z}\left(\Psi_{i}\left(x_{i}\right), \Psi\left(y^{\prime}\right)\right)=0$ and

$$
\lim _{i \rightarrow \infty} \frac{r_{i}\left|D \chi_{E}\right|\left(B_{r r_{i}}\left(x_{i}\right)\right)}{C\left(x, r_{i}\right)}=\left|D \chi_{F}\right|\left(B_{r}\left(y^{\prime}\right)\right), \quad \text { for } \mathscr{L}^{1} \text {-a.e. } r>0,
$$

where $C\left(x, r_{i}\right)$ was introduced in (1.24).

Let us set $X_{i}:=\Psi_{i}(X), E_{i}:=\Psi_{i}(E)$ and, with a slight abuse of notation, identity $F$ to $\Psi(F)$ and $y^{\prime}$ to $\Psi\left(y^{\prime}\right)$. Since by assumption it holds that $\left|D \chi_{E_{i}}\right| \rightarrow\left|D \chi_{F}\right|$, we have

$$
\lim _{i \rightarrow \infty}\left|D \chi_{E_{i}}\right|\left(B_{r}^{Z}\left(y^{\prime}\right)\right)=\left|D \chi_{F}\right|\left(B_{r}^{Z}\left(y^{\prime}\right)\right), \quad \text { for } \mathscr{L}^{1} \text {-a.e. } r>0 .
$$

This implies that the distance of $y^{\prime}$ from $X_{i}$ is infinitesimal as $i \rightarrow \infty$, hence we can find points $z_{i} \in X_{i}$ converging to $y^{\prime}$ in $Z$ satisfying

$$
\lim _{i \rightarrow \infty}\left|D \chi_{E_{i}}\right|\left(B_{r}^{Z}\left(z_{i}\right)\right)=\left|D \chi_{F}\right|\left(B_{r}^{Z}\left(y^{\prime}\right)\right), \quad \text { for } \mathscr{L}^{1} \text {-a.e. } r>0 .
$$

Let us set $x_{i}:=\Psi_{i}^{-1}\left(z_{i}\right)$. Observe that $\left|D \chi_{F}\right|\left(B_{r}^{Z}\left(y^{\prime}\right)\right)=\left|D \chi_{F}\right|\left(B_{r}^{Y}\left(y^{\prime}\right)\right)$ and

$$
\left|D \chi_{E_{i}}\right|\left(B_{r}^{Z}\left(z_{i}\right)\right)=\frac{r_{i}\left|D \chi_{E}\right|\left(B_{r r_{i}}\left(x_{i}\right)\right)}{C\left(x, r_{i}\right)},
$$

so that we get (A.3).

Let us now argue by contradiction. Assuming the conclusion of the lemma to be false we might find $\varepsilon>0$ such that the limit in (A.3) holds with $r=\varepsilon$ and

$$
B_{\varepsilon r_{i}}\left(x_{i}\right) \cap A=\emptyset \quad \text { for } i \text { sufficiently large, }
$$

with $x_{i}$ and $r_{i}$ as in (A.3). Let $M>0$ be large enough to grant that

$$
B_{\varepsilon r_{i}}\left(x_{i}\right) \subset B_{M r_{i}}(x)
$$

(it is simple to see that such a constant exists, since the convergence in $Z$ of $z_{i}=\Psi\left(x_{i}\right)$ ensures $\left.\mathrm{d}\left(x, x_{i}\right)=O\left(r_{i}\right)\right)$. Arguing as in the first part of the proof it is possible to see that

$$
\lim _{i \rightarrow \infty} \frac{r_{i}\left|D \chi_{E}\right|\left(B_{M r_{i}}(x)\right)}{C\left(x, r_{i}\right)}=\left|D \chi_{F}\right|\left(B_{M}\left(y^{\prime}\right)\right) \quad \text { for } \mathscr{L}^{1} \text {-a.e. } M>0
$$

and from now on we assume, possibly increasing $M$, that both (A.4) and (A.5) hold true. Then, in view of (A.4), we have

$$
\frac{\left|D \chi_{E}\right|^{*}\left(A \cap B_{M r_{i}}(x)\right)}{\left|D \chi_{E}\right|\left(B_{M r_{i}}(x)\right)}=\frac{\left|D \chi_{E}\right|^{*}\left(A \cap\left(B_{M r_{i}}(x) \backslash B_{\varepsilon r_{i}}\left(x_{i}\right)\right)\right)}{\left|D \chi_{E}\right|\left(B_{M r_{i}}(x)\right)} \leq 1-\frac{\left|D \chi_{E}\right|\left(B_{\varepsilon r_{i}}\left(x_{i}\right)\right)}{\left|D \chi_{E}\right|\left(B_{M r_{i}}(x)\right)} .
$$

Observe that the left hand side converges to 1 as $i \rightarrow \infty$, since $x$ is of density 1 for $A$. Therefore, to get the sought contradiction, it suffices to show that

$$
\liminf _{i \rightarrow \infty} \frac{\left|D \chi_{E}\right|\left(B_{\varepsilon r_{i}}\left(x_{i}\right)\right)}{\left|D \chi_{E}\right|\left(B_{M r_{i}}(x)\right)}>0
$$

Using (A.3) and (A.5), we get

$$
\liminf _{i \rightarrow \infty} \frac{\left|D \chi_{E}\right|\left(B_{\varepsilon r_{i}}\left(x_{i}\right)\right)}{\left|D \chi_{E}\right|\left(B_{M r_{i}}(x)\right)}=\frac{\lim _{i} \frac{r_{i}\left|D \chi_{E}\right|\left(B_{\varepsilon r_{i}}\left(x_{i}\right)\right)}{C\left(x, r_{i}\right)}}{\lim _{i} \frac{r_{i}\left|D \chi_{E}\right|\left(B_{M r_{i}}(x)\right)}{C\left(x, r_{i}\right)}} \geq \frac{\left|D \chi_{F}\right|\left(B_{\varepsilon}\left(y^{\prime}\right)\right)}{\left|D \chi_{F}\right|\left(B_{M}\left(y^{\prime}\right)\right)}>0,
$$

where the last inequality holds true since we are assuming that $y^{\prime} \in \operatorname{supp}\left|D \chi_{F}\right|$. 
Before passing to the proof of Theorem A.1 we need to introduce a definition and a lemma.

Definition A.3. We shall denote by $\mathcal{F}(K, N)$ the set of equivalence classes of quintuples $\mathfrak{X}=$ $(X, \mathrm{~d}, \mathfrak{m}, x, \nu)$ where $(X, \mathrm{~d}, \mathfrak{m}, x)$ is a pointed $\operatorname{RCD}(K, N)$ m.m.s and $\nu$ is a nonnegative and locally finite Borel measure with $\operatorname{supp} \nu \subset \operatorname{supp} \mathfrak{m}$, modulo the equivalence relation $\sim$ defined as follows. We say that $\left(X_{1}, \mathrm{~d}_{1}, \mathfrak{m}_{1}, x_{1}, \nu_{1}\right) \sim\left(X_{2}, \mathrm{~d}_{2}, \mathfrak{m}_{2}, x_{2}, \nu_{2}\right)$ if there exists an isometry $T:\left(\operatorname{supp} \mathfrak{m}_{1}, \mathrm{~d}_{1}\right) \rightarrow$ $\left(\operatorname{supp} \mathfrak{m}_{2}, \mathrm{~d}_{2}\right)$ such that $T_{\sharp} \mathfrak{m}_{1}=\mathfrak{m}_{2}, T\left(x_{1}\right)=x_{2}$ and $T_{\sharp} \nu_{1}=\nu_{2}$. We shall denote by $\mathcal{F}$ the union of the sets $\mathcal{F}(K, N)$ for $K \in \mathbb{R}, 1 \leq N<+\infty$. Observe that $\mathcal{F}$ can be realized as a countable union of sets $\mathcal{F}(K, N)$.

Let us introduce a distance in $\mathcal{F}$. Fix $\mathfrak{X}_{1}=\left(X_{1}, \mathrm{~d}_{1}, \mathfrak{m}_{1}, x_{1}, \nu_{1}\right), \mathfrak{X}_{2}=\left(X_{2}, \mathrm{~d}_{2}, \mathfrak{m}_{2}, x_{2}, \nu_{2}\right)$ in $\mathcal{F}$, a proper metric measure space $\left(Z, \mathrm{~d}_{Z}\right)$ and isometric embeddings $\Psi_{i}:\left(X_{i}, \mathrm{~d}_{i}\right) \rightarrow\left(Z, \mathrm{~d}_{Z}\right), i=1,2$. For any integer $n \geq 1$ we define

$$
\begin{aligned}
& \mathcal{D}_{n, \Psi_{1}, \Psi_{2}}\left(\mathfrak{X}_{1}, \mathfrak{X}_{2}\right):= \\
& \mathrm{d}_{H}\left(\Psi_{1}\left(X_{1} \cap \bar{B}\left(x_{1}, n\right)\right), \Psi_{2}\left(X_{2} \cap \bar{B}\left(x_{2}, n\right)\right)\right) \wedge 1 \\
& +\left|\log \left(\frac{\mathfrak{m}_{1}\left(B\left(x_{1}, n\right)\right)}{\mathfrak{m}_{2}\left(B\left(x_{2}, n\right)\right)}\right)\right| \wedge 1+W_{1}^{Z}\left(\left(\Psi_{1}\right)_{\sharp} \frac{\chi_{B\left(x_{1}, n\right)}}{\mathfrak{m}_{1}\left(B\left(x_{1}, n\right)\right)} \mathfrak{m}_{1},\left(\Psi_{2}\right)_{\sharp} \frac{\chi_{B\left(x_{2}, n\right)}}{\mathfrak{m}_{2}\left(B\left(x_{2}, n\right)\right)} \mathfrak{m}_{2}\right) \\
& +\left|\log \left(\frac{\nu_{1}\left(B\left(x_{1}, n\right)\right)}{\nu_{2}\left(B\left(x_{2}, n\right)\right)}\right)\right| \wedge 1+W_{1}^{Z}\left(\left(\Psi_{1}\right)_{\sharp} \frac{\chi_{B\left(x_{1}, n\right)}}{\nu_{1}\left(B\left(x_{1}, n\right)\right)} \nu_{1},\left(\Psi_{2}\right)_{\sharp} \frac{\chi_{B\left(x_{2}, n\right)}}{\nu_{2}\left(B\left(x_{2}, n\right)\right)} \nu_{2}\right) \text {, }
\end{aligned}
$$

where $\mathrm{d}_{H}$ is the Hausdorff distance between compact subsets of $Z$ and $W_{1}^{Z}$ is the 1-Wasserstein distance in $\left(Z, \mathrm{~d}_{Z} \wedge 1\right)$, namely

$$
W_{1}^{Z}(\mu, \nu):=\inf \left\{\int_{Z} \mathrm{~d}_{Z}(x, y) \wedge 1 \mathrm{~d} \pi(x, y): \pi \in \Gamma(\mu, \nu)\right\},
$$

with $\Gamma(\mu, \nu) \subset \mathscr{P}(X \times X)$ the set of probability measures having $\mu$ and $\nu$ as marginals. We finally define

$$
\mathcal{D}\left(\mathfrak{X}_{1}, \mathfrak{X}_{2}\right):=\inf _{\Psi_{1}, \Psi_{2}}\left\{\mathrm{~d}_{Z}\left(\Psi_{1}\left(x_{1}\right), \Psi_{2}\left(x_{2}\right)\right)+\sum_{n=1}^{\infty} \frac{1}{2^{n}} \mathcal{D}_{n, \Psi_{1}, \Psi_{2}}\left(\mathfrak{X}_{1}, \mathfrak{X}_{2}\right)\right\},
$$

the infimum being taken among all possible proper metric spaces $\left(Z, \mathrm{~d}_{Z}\right)$ and all isometric embed$\operatorname{dings} \Psi_{i}:\left(X_{i}, \mathrm{~d}_{i}\right) \rightarrow\left(Z, \mathrm{~d}_{Z}\right)$ for $i=1,2$.

Lemma A.4. $\mathcal{D}$ is a distance over $\mathcal{F}$ and a sequence $\left(X_{i}, \mathrm{~d}_{i}, \mathfrak{m}_{i}, x_{i}, \nu_{i}\right) \subset \mathcal{F}$ converges to $(Y, \varrho, \mu, y, \nu)$ in the topology induced by $\mathcal{D}$ if and only if $\left(X_{i}, \mathrm{~d}_{i}, \mathfrak{m}_{i}, x_{i}\right) \rightarrow(Y, \varrho, \mu, y)$ in the pmGH topology and $\nu_{i} \rightarrow \nu$ in duality with $\mathrm{C}_{\mathrm{bs}}(Z)$, where $\left(Z, \mathrm{~d}_{Z}\right)$ is a metric space where the pmGH convergence is realized. Moreover the subspace

$$
\overline{\mathcal{F}}:=\left\{(X, \mathrm{~d}, \mathfrak{m}, x, \nu) \in \mathcal{F}: \nu=h \mathfrak{m}, \text { with } h \in L^{\infty}(X, \mathfrak{m})\right\}
$$

is separable.

Proof. The verification that $\mathcal{D}$ is a distance is quite standard, see for instance [GMS15] . The equivalence between the two notions of convergence can be proved following the same strategy in the proof of [GMS15, Theorem 3.15], the only difference here being the addition to the quadruple of the measure $\nu$. Let us prove that $\overline{\mathcal{F}}$ is separable. It is enough to prove that, given $K$ and $N$, for any $k>0$ the set

$$
\overline{\mathcal{F}}_{k}(K, N):=\left\{(X, \mathrm{~d}, \mathfrak{m}, x, \nu) \in \mathcal{F}(K, N): \nu=h \mathfrak{m}, \text { with }\|h\|_{L^{\infty}(X, \mathfrak{m})} \leq k\right\}
$$

is compact. Let us fix a sequence $\left(X_{i}, \mathrm{~d}_{i}, \mathfrak{m}_{i}, x_{i}, \nu_{i}\right) \subset \overline{\mathcal{F}}_{k}(K, N)$. We can assume, up to extract a subsequence, that $\left(X_{i}, \mathrm{~d}_{i}, \mathfrak{m}_{i}, x_{i}\right) \rightarrow(Y, \varrho, \mu, y)$ in the pmGH topology. Let us fix a proper metric space $\left(Z, \mathrm{~d}_{Z}\right)$ realizing this convergence. Since $\nu_{i} \leq k \mathfrak{m}_{i}$ and $\mathfrak{m}_{i} \rightarrow \mu$ in duality with $\mathrm{C}_{\mathrm{bs}}(Z)$ we deduce that the measures $\nu_{i}$ are locally bounded in $Z$, uniformly in $i \in \mathbb{N}$. Therefore, possibly extracting a subsequence, there exists a positive measure $\nu$ in $Z$ such that $\nu_{i} \rightarrow \nu$ in duality with $\mathrm{C}_{\mathrm{bs}}(Z)$. It is immediate to check that $\nu \ll \mu$, with density uniformly bounded by $k$. This concludes the proof. 
We are ready to prove Theorem A.1.

Proof of Theorem A.1. Since tangents are invariant w.r.t. rescaling and closed w.r.t. $\mathcal{D}$-convergence, it is enough to prove that the set of points $x \in X$ such that there exist $(Y, \varrho, \mu, y, F) \in \operatorname{Tan}_{x}^{*}(X, \mathrm{~d}, \mathfrak{m}, E)$ and $y^{\prime} \in \operatorname{supp}\left|D \chi_{F}\right|$ such that

$$
\left(Y, \varrho, \mu_{y^{\prime}}^{1}, y^{\prime}, F\right) \notin \operatorname{Tan}_{x}(X, \mathrm{~d}, \mathfrak{m}, E)
$$

is $\left|D \chi_{E}\right|^{*}$-negligible, where $\mu_{1}^{y^{\prime}}:=C\left(y^{\prime}, 1\right)^{-1} \mu$ (see Definition 4.1).

Let us fix positive integers $k, m$ and a closed subset $\mathcal{U} \subset \overline{\mathcal{F}}$ with diameter, measured w.r.t. the distance $\mathcal{D}$ in (A.7), smaller than $(2 k)^{-1}$. Since, according to Lemma A.4, $\overline{\mathcal{F}}$ is separable, it is enough to prove that

$$
\begin{gathered}
A_{k, m}:=\left\{x \in X: \exists(Y, \varrho, \mu, y, F) \in \operatorname{Tan}_{x}^{*}(X, \mathrm{~d}, \mathfrak{m}, E) \cap \mathcal{U} \text { and } y^{\prime} \in \operatorname{supp}\left|D \chi_{F}\right|\right. \text { such that } \\
\left.\mathcal{D}\left(\left(Y, \varrho, \mu_{y^{\prime}}^{1}, y^{\prime}, F\right),\left(X, r^{-1} \mathrm{~d}, \mathfrak{m}_{x}^{r}, x, E\right)\right) \geq 2 k^{-1} \quad \forall r \in(0,1 / m)\right\}
\end{gathered}
$$

is $\left|D \chi_{E}\right|^{*}$-negligible, where we identified the set $F$ with the measure $\chi_{F} \mu$.

If, by contradiction, $\left|D \chi_{E}\right|^{*}\left(A_{k, m}\right)>0$, then, since $\left|D \chi_{E}\right|$ is asymptotically doubling by Proposition 4.8 , we can find $x \in A_{k, m}$ such that

$$
\lim _{r \downarrow 0} \frac{\left|D \chi_{E}\right|^{*}\left(A_{k, m} \cap B_{r}(x)\right)}{\left|D \chi_{E}\right|\left(B_{r}(x)\right)}=1,
$$

see (A.2). Since $x \in A_{k, m}$ there exist $(Y, \varrho, \mu, y, F) \in \operatorname{Tan}_{x}^{*}(X, \mathrm{~d}, \mathfrak{m}, E) \cap \mathcal{U}$ and $y^{\prime} \in \operatorname{supp}\left|D \chi_{F}\right|$ such that $\mathcal{D}\left(\left(Y, \varrho, \mu_{y^{\prime}}^{1}, y^{\prime}, F\right),\left(X, r^{-1} \mathrm{~d}, \mathfrak{m}_{x}^{r}, x, E\right)\right) \geq 2 k^{-1}$ for any $r \in(0,1 / m)$ and Lemma A.2 grants the existence of a sequence $\left(x_{i}\right) \subset A_{k, m}$ such that

$$
\lim _{i \rightarrow \infty} \mathrm{d}_{Z}\left(\Psi_{i}\left(x_{i}\right), \Psi\left(y^{\prime}\right)\right)=0,
$$

where $\Psi_{i}, \Psi$ are the embedding maps of Definition 1.20. Then, by definition of pmGH convergence, using the space $\left(Z, \mathrm{~d}_{Z}\right)$ we deduce

$$
\left(X, r_{i}^{-1} \mathrm{~d}, \mathfrak{m}_{x}^{r_{i}}, x_{i}\right) \rightarrow\left(Y, \varrho, \mu, y^{\prime}\right) .
$$

Since $\chi_{B^{Z}(\bar{z}, 1)}\left(1-\mathrm{d}_{Z}(\cdot, \bar{z})\right)$ belongs to $\mathrm{C}_{\mathrm{b}}(Z)$ for every $\bar{z} \in Z$, it is immediate to check that

$$
\left(X, r_{i}^{-1} \mathrm{~d}, \mathfrak{m}_{x_{i}}^{r_{i}}, x_{i}\right) \rightarrow\left(Y, \varrho, \mu_{y^{\prime}}^{1}, y^{\prime}\right), \quad \text { in the pmGH topology, }
$$

and $(\Psi)_{\#} \chi_{E} \mathfrak{m}_{x_{i}}^{r_{i}} \rightarrow \Psi_{\#} \chi_{F} \mu_{y^{\prime}}^{1}$ in duality with $\mathrm{C}_{\mathrm{bs}}(Z)$, that, thanks to (A.4), is equivalent to

$$
\mathcal{D}\left(\left(X, r_{i}^{-1} \mathrm{~d}, \mathfrak{m}_{x_{i}}^{r_{i}}, x_{i}, E\right),\left(Y, \varrho, \mu_{y^{\prime}}^{1}, y^{\prime}, F\right)\right) \rightarrow 0,
$$

see Definition A.3. Since $x_{i} \in A_{k, m}$ we can find $\left(Y_{i}, \varrho_{i}, \mu_{i}, y_{i}, F_{i}\right) \in \operatorname{Tan}_{x_{i}}^{*}(X, \mathrm{~d}, \mathfrak{m}, E) \cap \mathcal{U}$ and $y_{i}^{\prime} \in$ $\operatorname{supp}\left|D \chi_{F_{i}}\right|$ such that $\mathcal{D}\left(\left(Y_{i}, \varrho_{i},\left(\mu_{i}\right)_{y_{i}^{\prime}}^{1}, y_{i}^{\prime}, F_{i}\right),\left(X, r^{-1} \mathbf{d}, \mathfrak{m}_{x_{i}}^{r}, x_{i}, E\right)\right) \geq 2 k^{-1}$ for any $r \in(0,1 / m)$.

Using (A.10) and taking into account that by construction $\operatorname{diam} \mathcal{U}<(2 k)^{-1}$, we find the sought contradiction

$$
\begin{aligned}
2 k^{-1} & \leq \mathcal{D}\left(\left(Y_{i}, \varrho_{i},\left(\mu_{i}\right)_{y_{i}^{\prime}}^{1}, y_{i}^{\prime}, F_{i}\right),\left(X, r_{i}^{-1} \mathrm{~d}, \mathfrak{m}_{x_{i}}^{r_{i}}, x_{i}, E\right)\right) \\
& \leq \mathcal{D}\left(\left(Y, \varrho, \mu_{y^{\prime}}^{1}, y^{\prime}, F\right),\left(X, r_{i}^{-1} \mathrm{~d}, \mathfrak{m}_{x_{i}}^{r_{i}}, x_{i}, E\right)\right)+\mathcal{D}\left(\left(Y_{i}, \varrho_{i},\left(\mu_{i}\right)_{y_{i}^{\prime}}^{1}, y_{i}^{\prime}, F_{i}\right),\left(Y, \varrho, \mu_{y^{\prime}}^{1}, y^{\prime}, F\right)\right) \\
& \leq \mathcal{D}\left(\left(Y, \varrho, \mu_{y^{\prime}}^{1}, y^{\prime}, F\right),\left(X, r_{i}^{-1} \mathrm{~d}, \mathfrak{m}_{x_{i}}^{r_{i}}, x_{i}, E\right)\right)+(2 k)^{-1} \\
& \leq k^{-1}
\end{aligned}
$$

for $i$ large enough. 


\section{References}

[A97] L. Ambrosio: Corso introduttivo alla teoria geometrica della misura ed alle superfici minime. Appunti dei Corsi Tenuti da Docenti della Scuola. [Notes of Courses Given by Teachers at the School]. Scuola Normale Superiore, Pisa, (1997), ii+144.

[A01] L. Ambrosio: Some fine properties of sets of finite perimeter in Ahlfors regular metric measure spaces, Adv. Math., 159 (2001), 51-67.

[A02] L. Ambrosio: Fine properties of sets of finite perimeter in doubling metric measure spaces, Set-Valued Anal., 10 (2002), 111-128.

[A18] L. Ambrosio: Calculus, heat flow and curvature-dimension bounds in metric measure spaces, Proceedings of the ICM 2018, (2018).

[AF14] L. Ambrosio, J. Feng: On a class of first order Hamilton-Jacobi equations in metric spaces, J. Differential Equations, 256 (2014) 2194-2245.

[ADM14] L. Ambrosio, S. Di Marino: Equivalent definitions of BV space and of total variation on metric measure spaces. J. Funct. Anal., 266 (2014), 4150-4188.

[AGMr15] L. Ambrosio, N. Gigli, A. Mondino, T. Rajala: Riemannian Ricci curvature lower bounds in metric measure spaces with $\sigma$-finite measure. Trans. Amer. Math. Soc., 367 (2015), 4661-4701.

[AGS05] L. Ambrosio, N. Gigli, G. Savaré: Gradient flows in metric spaces and in the space of Probability measures. Lectures in Mathematics ETH Zurich, Birkhäuser, 2005.

[AgS14] L. Ambrosio, N. Gigli, G. Savaré: Metric measure spaces with Riemannian Ricci curvature bounded from below. Duke Math. J., 163 (2014), 1405-1490.

[AGS15] L. Ambrosio, N. Gigli, G. Savaré: Bakry-Émery curvature-dimension condition and Riemannian Ricci curvature bounds. Ann. Probab., 43 (2015), 339-404.

[AH17] L. Ambrosio, S. Honda: New stability results for sequences of metric measure spaces with uniform Ricci bounds from below. Measure Theory in Non-Smooth Spaces, De Gruyter Open, Warsaw, (2017), 1-51.

[AHT18] L. Ambrosio, S. Honda, D. Tewodrose: Short-time behavior of the heat kernel and Weyl's law on $\mathrm{RCD}^{*}(K, N)$-spaces. Ann. Global Anal. Geom., 53 (2018), 97-119.

[AKl08] L. Ambrosio, B. Kleiner, E. Le Donne: Rectifiability of sets of finite perimeter in Carnot groups: existence of a tangent hyperplane. J. Geom. Anal., 19 (2009), 509-540.

[AMS15] L. Ambrosio, A. Mondino, G. Savaré: Nonlinear diffusion equations and curvature conditions in metric measure spaces. ArXiv preprint: 1509.07273 (2015), to appear in Memoirs Amer. Math. Soc.

[AT14] L. Ambrosio, D. Trevisan: Well posedness of Lagrangian flows and continuity equations in metric measure spaces. Anal. PDE, 7 (2014), 1179-1234.

[BS18] E. Brué, D. Semola: Constancy of the dimension for $R C D(K, N)$ spaces via regularity of Lagrangian flows. To appear in Comm. Pure Appl. Math., ArXiv:1804.07128.

[C99] J. CheEger: Differentiability of Lipschitz functions on metric measure spaces. Geom. Funct. Anal., 9 (1999), 428-517.

[CM16] F. Cavalletti, E. Milman: The Globalization Theorem for the Curvature Dimension Condition, preprint on arXiv: 1612.07623.

[DG54] E. De GiongI: Su una teoria generale della misura $(r-1)$-dimensionale in uno spazio ad $r$ dimensioni, Ann. Mat. Pura Appl. (4) 36, (1954). 191-213. 
[DG55] E. De Giorgi: Nuovi teoremi relativi alle misure $(r-1)$-dimensionali in uno spazio ad $r$ dimensioni, Ricerche Mat. 4 (1955), 95-113.

[DPG16] G. De Philippis, N. Gigli: From volume cone to metric cone in the nonsmooth setting. Geom. Funct. Anal., 26 (2016), 1526-1587.

[DPG18] G. De Philippis, N. Gigli: Non-collapsed spaces with Ricci curvature bounded from below. J. Éc. polytech. Math., 5 (2018), 613-650.

[DPMR17] G. De Philippis, A. Marchese, F. Rindler: On a conjecture of Cheeger, Measure theory in non-smooth spaces, 145-155, Partial Differ. Equ. Meas. Theory, De Gruyter Open, Warsaw, 2017.

[EKS15] M. ERBAR, K. KuWADA, K.-T. Sturm: On the equivalence of the entropic curvaturedimension condition and Bochner's inequality on metric measure spaces. Invent. Math., 201 (2015), 993-1071.

[EGLS18] E.-B. Sylvester, T.-G. James, P. Lahti, N. Shanmugalingam: Asymptotic behavior of $B V$ functions and sets of finite perimeter in metric measure spaces, Preprint arXiv:1810.05310.

[FF60] H. Federer, H.-W. Fleming: Normal and integral currents, Ann. of Math. (2) 72 (1960) 458-520.

[F66] H.-W. Fleming: Flat chains over a finite coefficient group. Trans. Amer. Math. Soc., 121 (1966), 160-186.

[G13] N. Gigli: The splitting theorem in non-smooth context. Preprint arXiv:1302.5555.

[G14] N. GIGLI: An overview of the proof of the splitting theorem in spaces with non-negative Ricci curvature. Anal. Geom. Metr. Spaces, 2 (2014), 169-213.

[G15] N. GigLi: On the differential structure of metric measure spaces and applications. Mem. Amer. Math. Soc., 236 (2015), vi-91.

[G18] N. GigLi: Nonsmooth differential geometry: an approach tailored for spaces with Ricci curvature bounded from below. Mem. Amer. Math. Soc., 251 (2018), v-161.

[GH16] N. GigLi, B.-X. HAN: Independence on $p$ of weak upper gradients on RCD spaces. J. Funct. Anal., 271 (2016), 1-11.

[GMR15] N. Gigli, A. Mondino, T. RAJAla: Euclidean spaces as weak tangents of infinitesimally Hilbertian metric measure spaces with Ricci curvature bounded below. J. Reine Angew. Math., 705 (2015), 233-244.

[GMS15] N. Gigli, A. Mondino, G. Savaré: Convergence of pointed non-compact metric measure spaces and stability of Ricci curvature bounds and heat flows. Proc. Lond. Math. Soc. (3), 111 (2015), 1071-1129.

[GP16a] N. Gigli, E. Pasqualetto: Behaviour of the reference measure on RCD spaces under charts, preprint, arXiv:1607.05188.

[GP16b] N. Gigli, E. Pasqualetto: Equivalence of two different notions of tangent bundle on rectifiable metric measure spaces, Preprint arXiv:1611.09645.

[JLZ14] R. JiAng, H. Li, H. Zhang: Heat Kernel Bounds on Metric Measure Spaces and Some Applications. Potential Anal., 44 (2016), 601-627.

[KM18] M. Kell, A. Mondino: On the volume measure of non-smooth spaces with Ricci curvature bounded below, Ann. Sc. Norm. Super. Pisa Cl. Sci. (5) 18 (2018), no. 2, 593-610.

[LD11] E. Le Donne: Metric spaces with unique tangents. Ann. Acad. Sci. Fenn. Math., 36 (2011), 683-694. 
[LV09] J. Lott, C. Villani: Ricci curvature for metric-measure spaces via optimal transport. Ann. of Math. (2), 169 (2009), 903-991.

[M15] V. Magnani: On a measure theoretic area formula. Proc. Roy. Soc. Edinburgh Sect. A, 145 (2015), 885-891.

[Me01] X. Menguy: Examples of strictly weakly regular points. Geom. Funct. Anal., 11 (2001), 124-131.

[Mi03] M. Miranda JR.: Functions of bounded variation on "good" metric spaces. J. Math. Pures Appl., 82 (2003), 975-1004.

[MN14] A. Mondino, A. NABer: Structure theory of metric measure spaces with lower Ricci curvature bounds. ArXiv preprint 1405.2222 (2014), to appear on J. Eur. Math Soc.

[N14] A. NAKAYASU: Metric viscosity solutions for Hamilton-Jacobi equations of evolution type. Adv. Math. Sci. Appl., 24 (2014), 333-351.

[P11] A. Petrunin: Alexandrov meets Lott-Villani-Sturm. Münster J. Math., 4 (2011), 53-64.

[P87] D. PreIss: Geometry of measures in $\mathbb{R}^{n}$ : distributions, rectifiability and densities. Ann. of Math., 125 (1987), 537-643.

[S96] K.-T. Sturm: Analysis on local Dirichlet spaces. III. The parabolic Harnack inequality. J. Math. Pures Appl. (9), 75 (1996), 273-297.

[S06a] K.-T. Sturm: On the geometry of metric measure spaces I. Acta Math., 196 (2006), $65-131$.

[S06b] K.-T. Sturm: On the geometry of metric measure spaces II. Acta Math., 196 (2006), 133-177.

[T15] X. Tolsa: Characterization of n-rectifiability in terms of Jones' square function: part I. Calc. Var. \& PDE, 54 (2015), 3643-3665.

[AT15] J. Azzam, X. Tolsa: Characterization of n-rectifiability in terms of Jones' square function: Part II. Geometric and Functional Analysis, 25 (2015), 1371-1412.

[V09] C. Villani: Optimal transport. Old and New. Grundlehren der Mathematischen Wissenschaften, 338. Springer-Verlag Berlin, 2009.

[VR08] M.-K. Von Renesse: On local Poincaré via transportation. Math. Z., 259 (2008), 2131.

[We00] N. Weaver: Lipschitz algebras and derivations. II. Exterior differentiation. J. Funct. Anal., 178 (2000), 64-112.

[W89] B. White: A new proof of the compactness theorem for integral currents. Comm. Math. Helvetici, 64 (1989), 207-220. 